\begin{abstract}
Title of Document:

MODELING AND EXPERIMENTS ON INJECTION INTO UNIVERSITY OF MARYLAND ELECTRON RING
\end{abstract}

Gang Bai, Master of Science, 2005

Directed By:

Professor Patrick G. O'Shea

Department of Electrical and Computer Engineering

The University of Maryland Electron Ring (UMER) is built as a low-cost testbed for intense beam physics for benefit of larger ion accelerators. The beam intensity is designed to be variable, spanning the entire range from low current operation to highly space-charge-dominated transport. The ring has been closed and multi-turn commissioning has begun. One of the biggest challenges of multi-turn operation of UMER is correctly operating the Y-shaped injection/recirculation section, which is specially designed for UMER multi-turn operation. It is a challenge because the system requires several quadrupoles and dipoles in a very stringent space, resulting in mechanical, electrical, and beam control complexities. Also, the earth's magnetic field and the image charge effects have to be investigated because they are strong enough to impact the beam centroid motion. This thesis presents both simulation and experimental study of the beam centroid motion in the injection region to address above issues. 


\title{
MODELING AND EXPERIMENTS ON INJECTION INTO UNIVERSITY OF MARYLAND ELECTRON RING
}

\author{
By \\ Gang Bai \\ Thesis submitted to the Faculty of the Graduate School of the \\ University of Maryland, College Park, in partial fulfillment \\ of the requirements for the degree of \\ Master of Science \\ 2005
}

Advisory Committee:

Professor Patrick O’Shea, Chair/advisor

Professor Martin Reiser

Professor Rami Kishek 
(C) Copyright by Gang Bai 2005 


\section{Dedication}

To my family with all my love. 


\section{Acknowledgements}

Here please let me thank everyone who has helped me on my study and research in University of Maryland until now. I have learned much from you and am honored to continue my study among you in the future.

Thank you Professor Patrick O'Shea for giving me the opportunity to continue my education and for your continuous guidance on my study and research. I am very thankful for finding an advisor who is nice and understands students.

Thank you Professor Martin Reiser for your kind instructions on my project, patient explanation on the theory and encouraging me to improve my English.

Thank you Professor Rami Kishek for all of your guidance, assistance and patience. I am forever in debt with you.

Thank you Dr. Walter, Dr. Bernal, Mr. Bryan Quinn for your great help with my research.

Thank you Dr. Haber, Dr. Godlove, Dr. Feldman and Dr. Sutter for your kind assistance on my project.

Special thanks for Mrs. Renee Feldman who revised my master thesis; thanks for T. Firestone and N. Moody for kind assistance on my writing.

Thank you the graduate students in UMER group and IREAP.

Also thank the U.S. Department of Energy (DOE), who supports the UMER project.

Thank you my family for your unconditional love and support for my life. 


\section{Table of Contents}

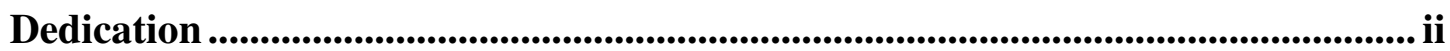

Acknowledgements ...................................................................................................................ii

Table of Contents ........................................................................................................... iv

List of Tables .................................................................................................................. vi

List of Figures.............................................................................................................................. vii

Chapter 1: Introduction .............................................................................................................. 1

1.1 Background and motivation ......................................................................... 1

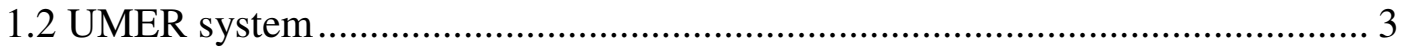

1.3 Organization of the thesis ........................................................................... 10

Chapter 2: Improved Modeling on Y-section............................................................... 13

2.1 Introduction of injection/recirculating section................................................ 13

2.2 Improved model and numerical calculations for Y-section ............................... 15

2.2.1 Key magnets' modeling in the improved model....................................... 15

2.2.2 Numerical calculation for tracking the beam centroid in the improved

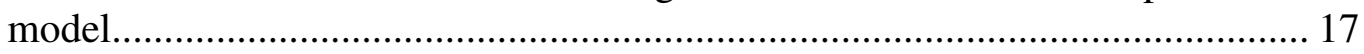

2.2.3 Comparison of key magnet settings calculated from the improved model to previous work

2.2.4 Beam trajectories and key magnet settings with different modeling for the pulsed dipole and earth's field ......................................................................... 23

2.3 Comparison of magnets' settings in the improved model with different modeling of the PD thickness and the earth's field ............................................... 28

Chapter 3 More Accurate Modeling and Calculations on Y- Section .................... 30

3.1 More accurate and extended magnetic modeling from IC2 to RC2 ................ 30

3.1.1 Layout of the extended magnetic model .................................................... 30

3.1.2 Modeling of key magnets in the more accurate and extended magnetic

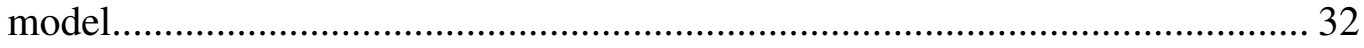

3.1.3 Calculation of more accurate effective lengths of big magnets in the overlapped field at Y-section.......................................................................... 35

3.2 Numerical calculation of beam centroid with Matlab in the more accurate

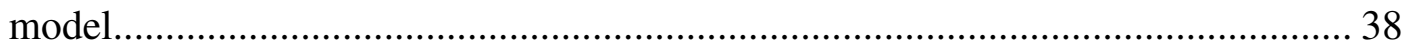

3.2.1 Leap-frog iteration method for beam centroid calculations........................ 38

3.2.2 Beam centroid calculations based on the more accurate and extended

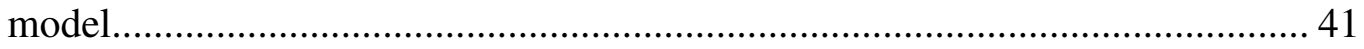

3.3 Study of beam centroid motion at RC2 due to the steering dipole strength

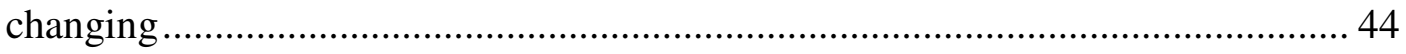

3.3.1 Sensitivity study with Matlab simulation model ........................................ 44

3.3.2 Sensitivity study in WARP simulation containing space charge................ 46

3.3.3 Comparison and analysis of simulation results between Matlab and WARP

3.4 Calculation of the pulsed dipole settings based on the more accurate simulation

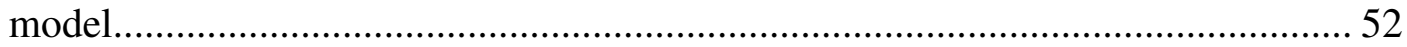

3.4.1 Beam centroid trajectory for one turn in the ring with WARP simulation 52 
3.4.2 Study on the pulsed dipole setting when the beam injects into the ring .... 54

3.4.3 Returning leg study based on the more accurate model ......................... 57

3.4.4 Comparison between Matlab simulation results on the pulsed dipole settings for injecting/returning beam with the experiment ............................. 59

Chapter 4 Experiment Study on Y-section .............................................................. 61

4.1 Experiment setup for study of injection/recirculating section ........................ 61

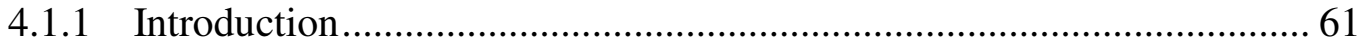

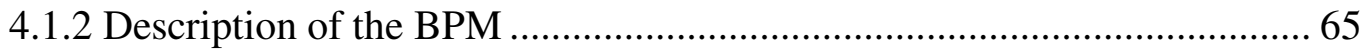

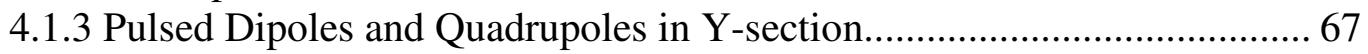

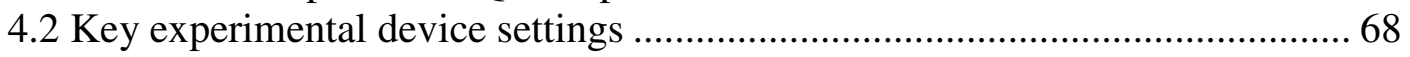

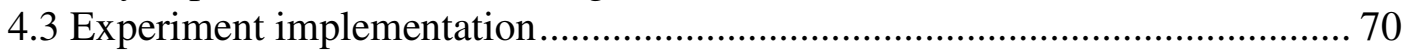

4.3.1 Obtain initial beam centroid $X$ position and angle ............................... 71

4.3.2 Experimental measurement of sensitivity of beam centroid at RC2 2........ 74

4.4 Experiment result analysis and comparison with simulations ......................... 76

4.4.1 Import the beam initial centroid into Matlab model ............................... 76

4.4.2 WARP simulation study on the beam centroid sensitivity ....................... 80

4.4.3 Analysis and comparison of experiment, Matlab, WARP simulation results

Chapter 5 Conclusion ................................................................................................. 85

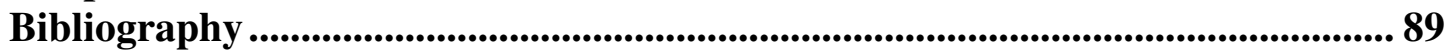




\section{List of Tables}

Table 1.1 Comparison of key parameters for electron beam and heavy ion beam ....... 4

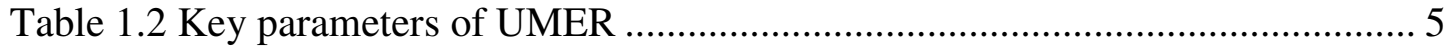

Table 1.3 Beam parameters with different beam currents .......................................... 6

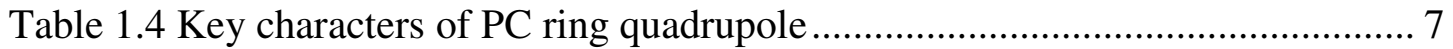

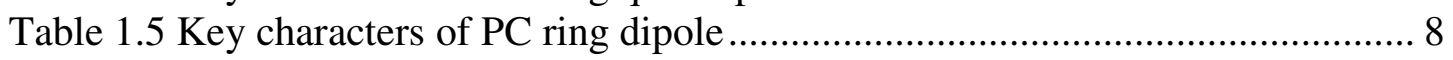

Table 2.1 Comparison of Model 1 calculation results to previous work .................... 23

Table 2.2 Comparison of magnet settings in the improved model on different

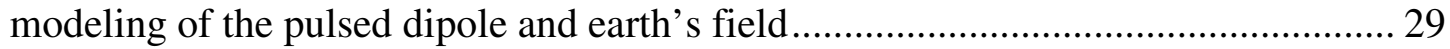

Table 3.1 Layout details of magnets in the extended model of Y-section................... 31

Table 3.2 Earth's fields at different locations along the model ................................ 35

Table 3.3 More accurate effective lengths of magnets overlapped in Y-section........ 37

Table 3.4 WARP simulation parameters for beams with/without space charge......... 47

Table 3.5 Sensitivities of beam centroid for SD5h and SD6h scans in Matlab and WAPR simulations.

Table 3.6 Settings of the pulsed dipole for injecting and returning beam in experiment

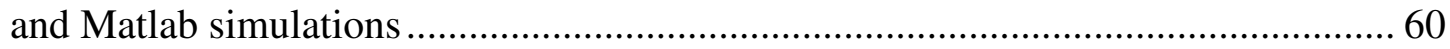

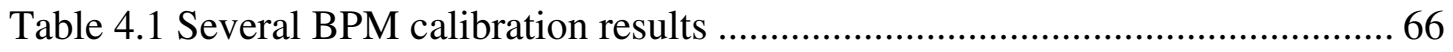

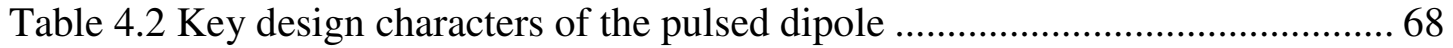

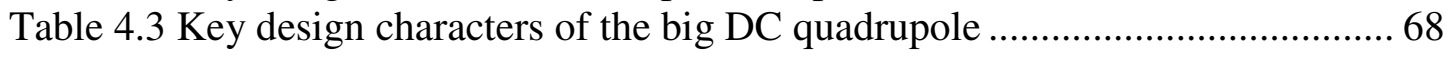

Table 4.4 Several important experimental settings ................................................. 70

Table 4.5 Experimental settings of involved magnets ............................................ 74

Table 4.6 Bending angles of involved dipoles set in the Matlab simulation corresponding to experimental settings 78

Table 4.7 Centroid sensitivities of beam at RC2 for SD5h and SD6h scans among experiments, Matlab and WAPR simulations 


\section{List of Figures}

Figure 1.1 Layout of University of Maryland Electron Ring (UMER) ...................... 5

Figure 1.2 Ring FODO layout and wire patterns of PC dipole and quadrupole .......... 7

Figure 1.3 Measured earth's field at UMER location............................................... 9

Figure 1.4 UMER Injection/recirculating section................................................. 10

Figure 2.1 Progress of the pulsed dipole operation................................................ 14

Figure 2.2 Layout of UMER injection/recirculating section ................................ 15

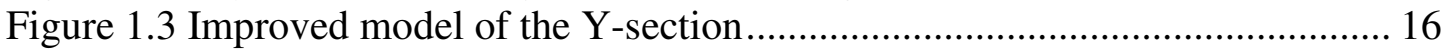

Figure 2.4 Beam trajectory in Model 1: with thin PD/without earth field.................. 22

Figure 2.5 Beam trajectory in Model 2: with thick PD/without earth field ............... 24

Figure 2.6 Beam trajectory in Model 3: with thick PD \& with earth field ................. 25

Figure 2.7 Comparison of beam centroid trajectories in WARP frame: with thick PD,

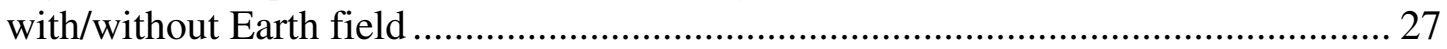

Figure 2.8 Beam X centroid and envelopes for one turn in WARP simulation.......... 28

Figure 3.1 Layout of more accurate and extended model for Y-section ................... 31

Figure 3.2 Overlapped magnetic fields of big magnets in the restricted Y region ..... 36

Figure 3.3 Magnetic fields on the pipe center in the Y-section involved with

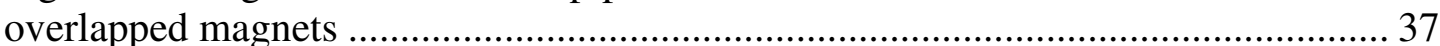

Figure 3.4 Beam centroid in the more realistic Y-section model with WARP

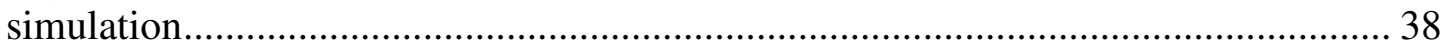

Figure 3.5 Schematic diagram of beam positions calculation in the more accurate

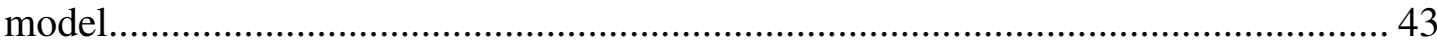

Figure 3.6 Sensitivity of the beam centroid at RC2 due to the SD5h scan in the

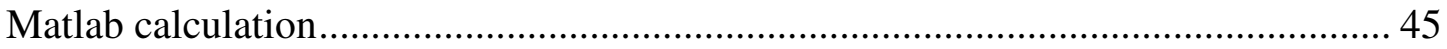

Figure 3.7 Sensitivity of the beam centroid at RC2 due to the SD6h scan in the

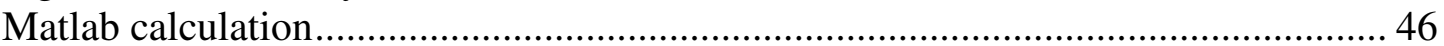

Figure 3.8 Sensitivity of the beam centroid at RC2 due to the SD5h scan in the

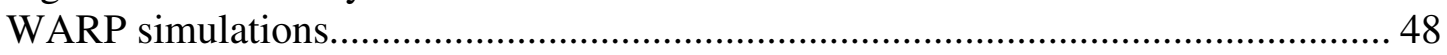

Figure 3.9 Sensitivity of the beam centroid at RC2 due to the SD6h scan in the

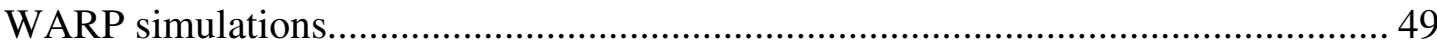

Figure 3.10 Best fit analysis and comparison of the beam centroid at RC2 due to SD5h scan between Matlab and WARP simulations ............................................ 50

Figure 3.11 Best fit analysis and comparison of the beam centroid at RC2 due to SD6h scan between Matlab and WARP simulations ............................................. 50

Figure 3.12 Beam centroid for one turn of the ring with WARP simulation............. 53 Figure 3.13 Schematic beam centroid trajectories for injection and recirculation cases

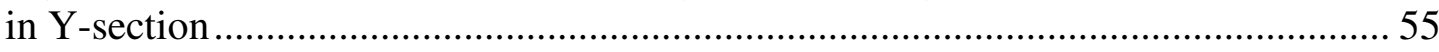
Figure 3.14 Beam centroid in the ring part passing through centers of QR1 and QR2

Figure 3.15 Schematic layout of returning leg for the calculation pf PD setting for

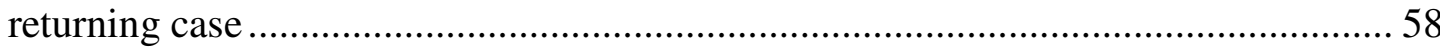

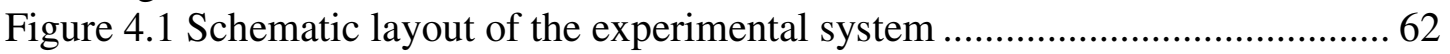

Figure 4.2 Layout of a ring chamber with BPM and P-screen housed ..................... 65 


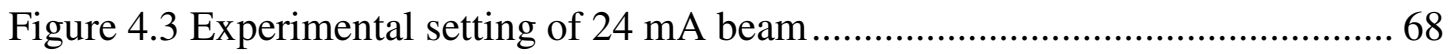

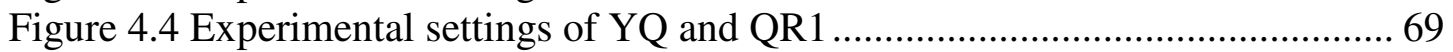

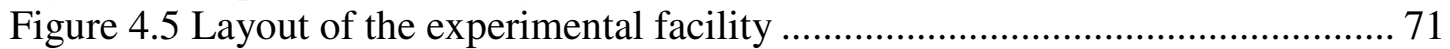

Figure 4.6 Typical quadrupole scan and results showing the beam centering the

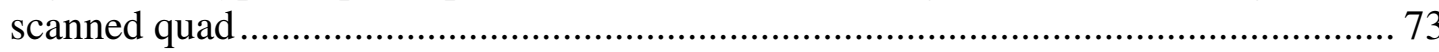

Figure 4.7 (a) Centroid sensitivity of $24 \mathrm{~mA}$ beam at RC2 due to the SD5h scan in the experiment (b) Centroid sensitivity of $24 \mathrm{~mA}$ beam at RC2 due to the SD6h scan in

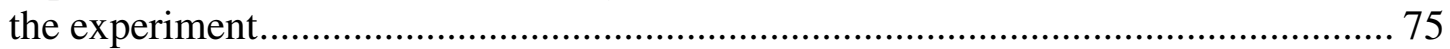

Figure 4.8 (a) Centroid sensitivity of the beam at RC2 due to the SD5h scan in Matlab calculation (b) Centroid sensitivity of the beam at RC2 due to the SD6h scan in Matlab calculation........................................................................................ 79

Figure 4.9 (a) Beam centroid sensitivities at RC2 due to the SD5h scan in WARP simulations (b) Beam centroid sensitivities at RC2 due to the SD6h scan in WARP

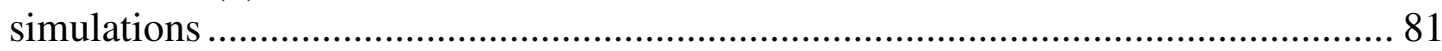

Figure 4.10 Best fit analysis and comparison of the beam centroid at RC2 due to SD5h scan among experiments, Matlab and WARP simulations............................ 82

Figure 4.11 Best fit analysis and comparison of the beam centroid at RC2 due to SD6h scan among experiments, Matlab and WARP simulations 


\section{Chapter 1: Introduction}

\section{$\underline{1.1 \text { Background and motivation }}$}

In recent years, the technology of intensive beams had extensive applications in the domains of industry, agriculture and medical devices. The applications of high intensity beam include spallation neutron sources [1], free electron lasers [2], and heavy-ion fusion drivers $[3,4,5]$.

For all these applications, the beam centroid steering is the first step to achieve optimal beam control [6]. The centroid steering precedes other considerations such as beam matching, space charge effect, imaging force, etc. To successfully steer the beam centroid in the designed orbit, the physics issues of bending magnets (dipoles), external focusing magnets (quadrupoles and solenoids) and self fields (space charges), must be studied.

The University of Maryland Electron Ring (UMER) is a special, low-cost, scaled, electron storage ring designed for research in intensive beam physics in order to understand the physics of applications of intensive beams [7, 8, 9]. More details on UMER can be found in Section 1.2.

The centroid steering is more complicated on the UMER's case. The complexities reside in: (a) the electron beam operated by UMER is a low-energy electron beam of $10 \mathrm{keV}$, as to be affected by the earth's magnetic field even varying at different locations in the UMER laboratory; (b) One of the biggest challenges of the beam steering in UMER is correctly implementing the injection/recirculating 
scheme required by the beam multi-turn operation. In UMER, the injection/recirculating section is called Y-section according to its shape. Please refer to Section 1.2.3 for details of the Y-section. Since the electron beam transported in UMER is a $100 \mathrm{~ns}$ long pulse, which is long enough to occupy half the ring. We have a window of less than $100 \mathrm{~ns}$ after injection to flip the polarity of the single pulsed dipole, PD, in time for recirculating the injected pulse. Furthermore, according to the matched beam envelop equation and zero-current phase advance limit for stability issue, the system requires a short lattice period for UMER. This results in that a single quadrupole, YQ (Y quadrupole), the PD and the other single quadrupole, QR1, are squeezed in a stringent space, less than $20 \mathrm{~cm}$. Also, the YQ is shared between the injection line and the returning leg, assisting the PD to execute injecting and recirculating because of its off-centered from both injection line and returning leg. The multi-turn operation requires accurate designs of the Y-section magnets, involving with a study of the complicated, overlapped magnetic field in the Y-section due to the layouts of the magnets there. Moreover, the entire Y-section in UMER is blind (i.e. has no diagnostics), making beam-based steering difficult.

The complexity of the injection/recirculation scheme for UMER multi-turn beam control plus the unavoidable effect from the earth's magnetic field makes correct design of the Y-section for UMER multi-turn operation an interesting and challenging task. Dr. H. Li has been able to derive analytic equations for determining the required magnet settings for the Y-section [6]. More realistic operation conditions, however, such as the presence of the earth's magnetic field, thick magnets that overlap with each other, and machine errors, add more complexity to the 
calculation [10]. These issues are addressed in this thesis using semi-analytic numerical models. More accurate modeling of the magnetic field will let us obtain more accurate solutions of magnet settings. The setting of the switch magnet for beam injecting or returning and the difference of settings for these two cases caused by asymmetric the earth's field effect are worth studying. The motion of the beam centroid passing through the Y-section is also studied. We are concerned with the linearity of the beam centroid at a location due to the beam initial condition variation; the image charge effect on the beam motion. In order to address space charge effect [11], we use the self-consistent PIC simulation with WARP $[12,13]$, the results of which are compared to the numerical Matlab model and experiments. The results from the calculations and experiments allow us to rank these effects in order of importance, and point out which can be neglected and which can not.

\subsection{UMER system}

UMER actually is a simulator on a much smaller scale, for advanced accelerators and high intense beam storages, which is really huge and a money-burner compared with the UMER facility. The electron beams provided by UMER can simulate the behavior of heavy ion beams because both have almost the same $\beta$ (velocity of particles/velocity of light), generalized perveance $\mathrm{K}$, and intensity parameter $\chi$ [14]. Some characters for these two kinds of beam are shown in Table 1.1. So the UMER facility is constructed on a much smaller scale and is less expensive to provide guidance for high intensity and energy beam research $[14,15]$. 
Table 1.1 Comparison of key parameters for electron beam and heavy ion beam

\begin{tabular}{|c|c|c|}
\hline Beam parameters & Heavy ion beam & Electron beam \\
\hline Energy & $10 \mathrm{GeV}$ & $10 \mathrm{keV}$ \\
\hline$\beta=\mathrm{v} / c$ & 0.3 & 0.2 \\
\hline Current & $5 \mathrm{kA}$ & $100 \mathrm{~mA}$ \\
\hline Mass & $3.7 * 10^{5} \mathrm{~m}_{\mathrm{e}}$ & $\mathrm{m}_{\mathrm{e}}$ \\
\hline Charge state & +1 & -1 \\
\hline Generalized perveance $\mathrm{K}$ & $8.7 * 10^{-4}$ & $1.5^{*} 10^{-3}$ \\
\hline
\end{tabular}

Figure 1.1 indicates the layout of UMER, which now is closed for multi-turn study. UMER facility is composed mainly of electron beam source (electron gun), injection line, injection/circulating section, and the main ring. The electron beams provided by UMER are low energy of $10 \mathrm{keV}, 100 \mathrm{~ns}$ long pulse, rectangular beams. The main characteristics of UMER are shown in Table 1.2. The beam current is varied from $0.6 \mathrm{~mA}$ to $100 \mathrm{~mA}$ by adjusting the collimating apertures according to the different requirements of specific experiments. Usually, smaller current beams are used in beam steering and transport, while higher current beams are used in the research of space charge effect. Table 1.3 shows some typical beams with different beam currents and corresponding characteristics such as emittance and phase advance. We can see that UMER facility can provide low energy electron beams with extensive currents and $\chi$. 


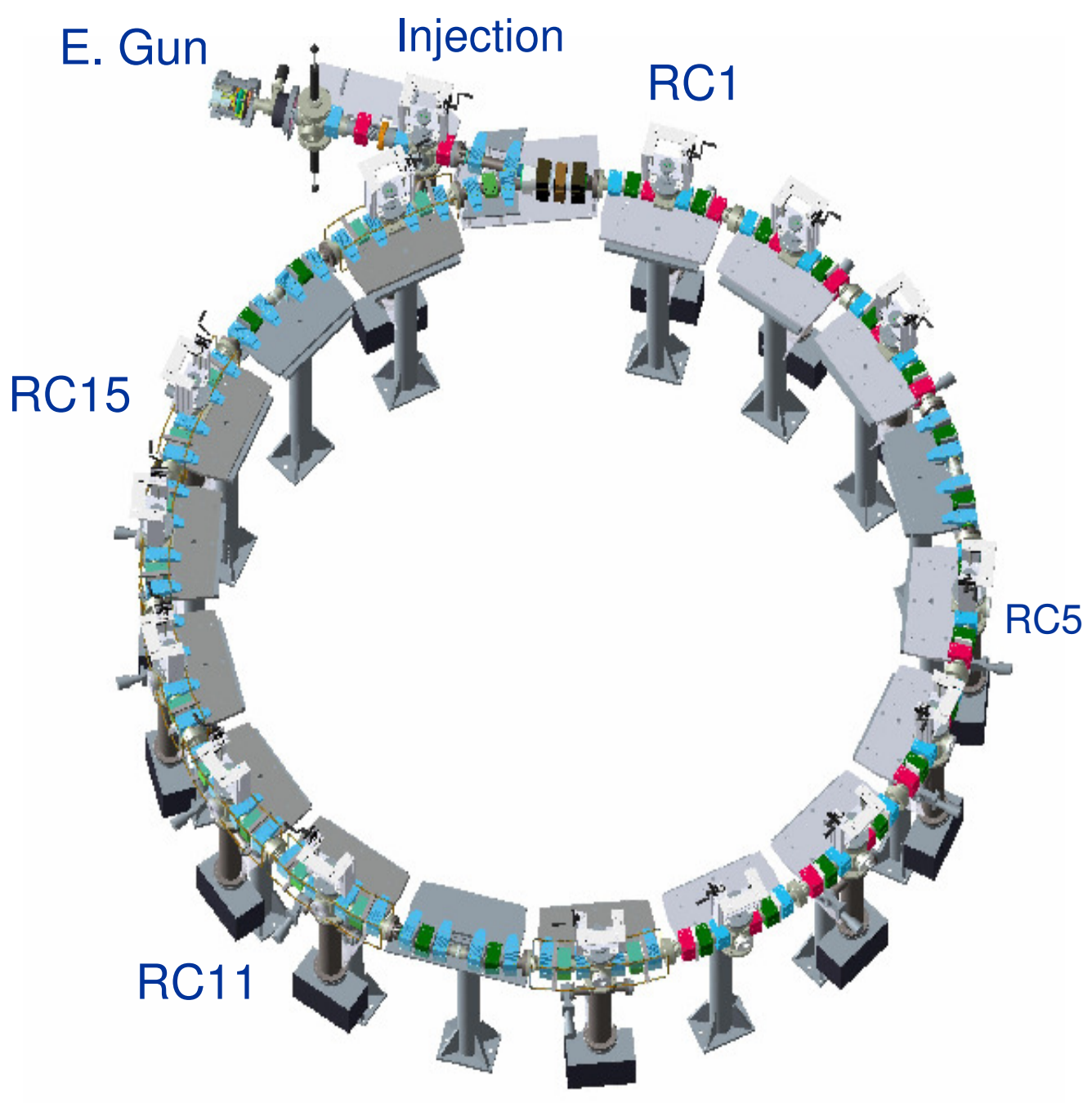

Figure 1.1 Layout of University of Maryland Electron Ring (UMER)

Table 1.2 Key parameters of UMER

\begin{tabular}{|ll}
\hline Main beam current & $0.6-100 \mathrm{~mA}$ \\
Electron beam energy & $10 \mathrm{keV}$ \\
Main beam emittance(4×rms, unnormalized) & $60 \mathrm{~mm} \cdot \mathrm{mrad}$ \\
Circumference & $11.52 \mathrm{~m}$ \\
Pulse length & $100 \mathrm{~ns}$ \\
Lattice period & $32 \mathrm{~cm}$ \\
Zero-current phase advance per FODO $\left(\sigma_{0}\right)$ & $76^{\circ}$ \\
Betatron tune depression & $>0.16$ \\
\hline
\end{tabular}


Table 1.3 Beam parameters with different beam currents

\begin{tabular}{|cccccc|}
\hline$I(\mathrm{~mA})$ & $\varepsilon(\mathrm{mm} \cdot \mathrm{mr})$ & $a_{0}(\mathrm{~mm})$ & $a(\mathrm{~mm})$ & $\chi$ & $\sigma \sigma_{0}$ \\
100 & 60 & 3.2 & 9.5 & 0.98 & 0.16 \\
24 & 30 & 1.5 & 4.8 & 0.90 & 0.31 \\
7 & 15 & 0.875 & 2.8 & 0.78 & 0.47 \\
0.6 & 5.5 & 0.25 & 1.3 & 0.32 & 0.82 \\
\hline
\end{tabular}

$\left(a_{0}:\right.$ beam size at the aperture plate; $a:$ average matched beam size in the ring; $\varepsilon: 4 \times \mathrm{rms}$, unnormalized emittance)

The main focusing lattice of UMER is made of 18 sections. Each section includes two equal lattices, each of them $32 \mathrm{~cm}$ long and with a bend angle of $10^{\circ}$. The total is $360^{\circ}$. Between two lattices in a section, a diagnostics chamber, in which a BPM (beam position monitor) and a phosphor screen are housed, is set up at the midpoint of the section. (We will give a detailed introduction of the beam diagnostics chamber in Chapter 4) The circumference of the whole ring is $11.52 \mathrm{~m}$. One periodic lattice is made of two symmetric quadrupoles but one is defocusing while the other is focusing according to design requirements, forming a FODO, and a bending dipole is located at the midpoint of a lattice. All of the magnets in each FODO are printed circuit (PC) magnets. The FODO schematic layout and the wire pattern of PC quadrupoles and PC dipoles are shown in Figure 1.2. The design of these short PC magnets follows the principles of the design of Lambertson magnets $[16,17]$. These PC magnets have been characterized with a Rawson-Lush rotating coil [18]. The details are referred to previous work $[19,20]$. 


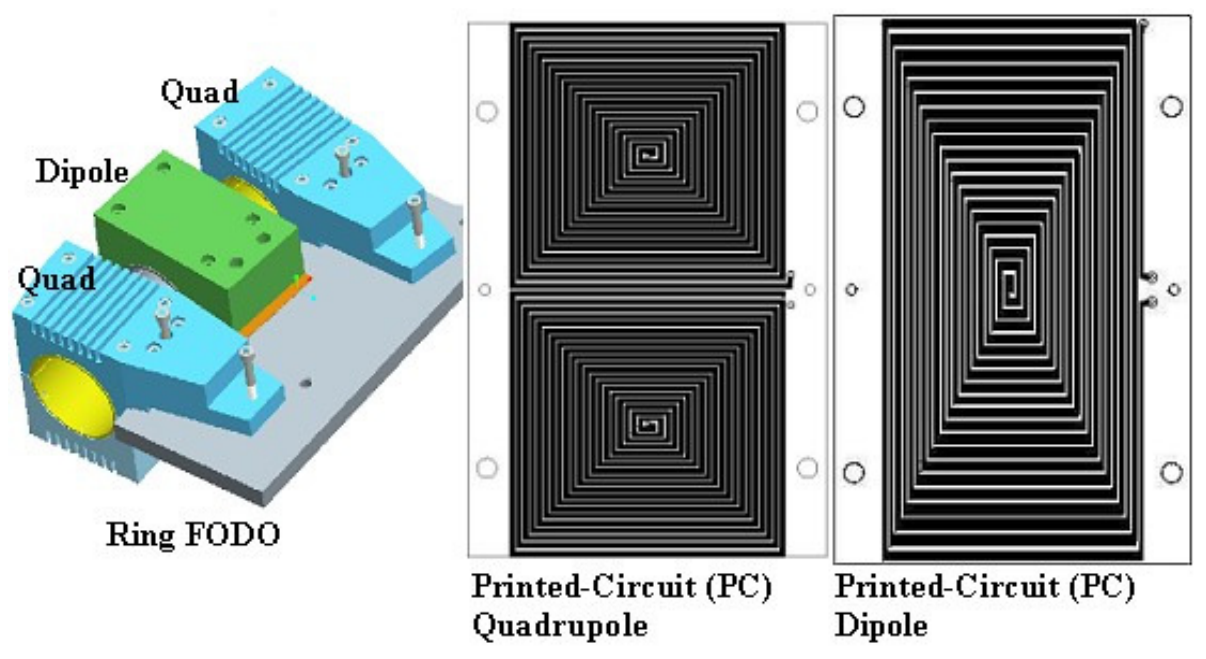

Figure 1.2 Ring FODO layout and wire patterns of PC dipole and quadrupole

In a lattice, two quadrupoles and one bending dipole divide one period into four equal segments, each $8 \mathrm{~cm}$. The two kinds of quadrupoles are main focusing magnets in the UMER lattice. For the printed circuit design, the adjustment of the quadrupole strength is flexible because the currents can reach $3.5 \mathrm{~A}$ and also the polarity can be reversed. The normal operation current of the quadrupole is $1.88 \mathrm{~A}$ and the power consumption is around $12 \mathrm{~W}$ so it is easy to find a standard power supply for quadrupole operation. The main characteristics of the quadrupole are shown in Table 1.4.

Table 1.4 Key characters of PC ring quadrupole

\begin{tabular}{ll}
\hline Field gradient & $4.1 \mathrm{G} / \mathrm{cm} / \mathrm{A}$ \\
Current & $2 \mathrm{~A}$ \\
Physical length & $4.4 \mathrm{~cm}$ \\
effective length & $3.6 \mathrm{~cm}$ \\
Radius & $2.8 \mathrm{~cm}$ \\
Field integral & $15 \mathrm{G} / \mathrm{A}$ \\
Resistance (room temp.) & $7 \Omega$ \\
Allowed harmonic content & $<1 \%$ \\
Transverse alignment error & $<0.05 \mathrm{~mm}$ \\
\hline
\end{tabular}


The ring dipole in a period works as a bending magnet in the ring. According to the ring design, one periodic lattice should bend the beam $10^{\circ}$ for a total of $360^{\circ}$. Since the vertical component of the earth's field at the location of UMER facility is around 0.4 Gauss (Helmholtz coils are used along the whole ring to cancel the horizontal earth's field), the earth's field can bend the electron beam in the horizontal plane to the right with approximate $2^{\circ}$ along one period. So the ring dipole is set around $2.4 \mathrm{~A}$ to bend the beam $8^{\circ}$ horizontally in a FODO. On the other hand, 18 ring vertical steering dipoles are distributed along the ring for deflecting the beam with smaller angles, compared with the bending angles of the ring dipoles in the horizontal plane (we will discuss the steering dipoles in detail in Chapter 4). Table 1.5 gives the main characters of the ring dipole.

Table 1.5 Key characters of PC ring dipole

\begin{tabular}{ll}
\hline Dipole field & $5.2 \mathrm{G} / \mathrm{A}$ \\
Current & $3 \mathrm{~A}$ \\
Physical length & $4.4 \mathrm{~cm}$ \\
Effective length & $3.8 \mathrm{~cm}$ \\
Radius & $2.8 \mathrm{~cm}$ \\
Field integral & $19.61 \mathrm{Gcm} / \mathrm{A}$ \\
Resistance & $3 \Omega$ \\
Allowed harmonic content & $<1 \%$ \\
Transverse alignment error & $<0.05 \mathrm{~mm}$ \\
\hline
\end{tabular}

The earth's field varies along the ring at different locations, the measurements of which are shown in Figure 1.3 [21]. The angles on horizontal axis represent the locations along the ring circle. The red squares represent the vertical component of the earth's field, which fluctuates around $-400 \mathrm{mG}$. The negative sign means the field pointing down; the green squares represent the earth's field component on the 
direction of beam transport along the ring; the earth's field component on the radial direction in the horizontal plane is represented by the blue squares, fluctuating between $\pm 200 \mathrm{mG}$. The positive sign applies the field towards the ring center. From the measurements, the electron beam centroid is bent "to right" in the horizontal plane everywhere along the ring, by the vertically down component of the earth's field. That is why UMER is initially designed to operate the beam turning "to right" to utilize the earth's field vertical component.

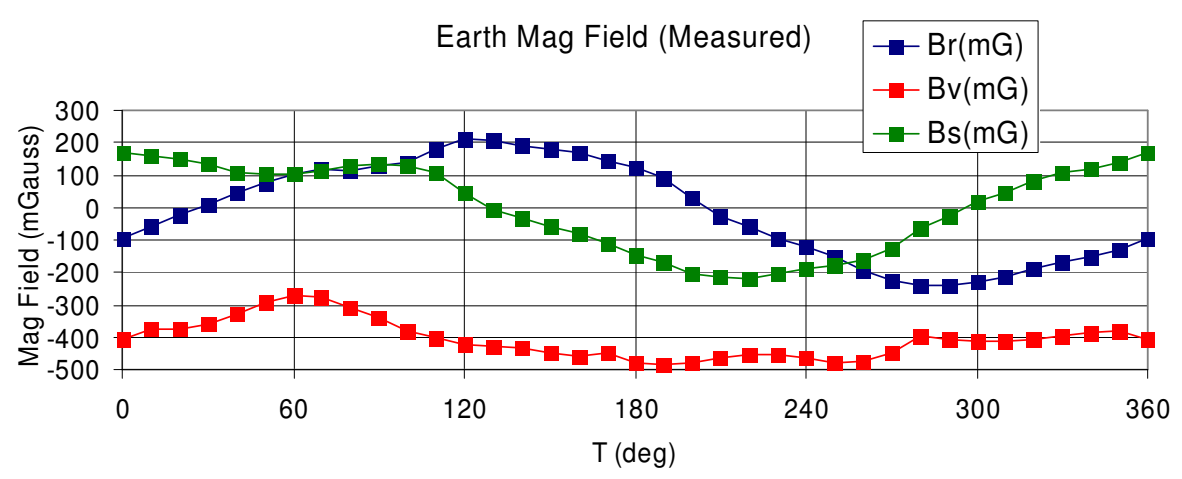

Figure 1.3 Measured earth's field at UMER location

Figure 1.4 is a Pro-E drawing of the inflecting/recirculating section (Ysection), used for UMER. It is composed of the injection line (the flange upstream Q5 to PD), joining the returning leg (the flange upstream QR70 to PD), each making a $10^{\circ}$ angle with the ring line (PD to the flange downstream $\mathrm{QR} 1$ ). The shared big quadrupole, YQ, is off-centered from the reference trajectories of the beam, hence plays a part in bending the beam towards the injector. The YQ is designed as horizontal defocusing. When the beam is injected, hitting the left part of the YQ, the beam is bent more to the left in the horizontal plane; similarly, the beam centroid is deflected more to the right horizontally when the beam returns from the ring. Thus 
the YQ always helps steer the beam into the ring for both injection and returning beams. Additional steering dipoles, SD6h, are added upstream to help steer the beam in the horizontal plane.

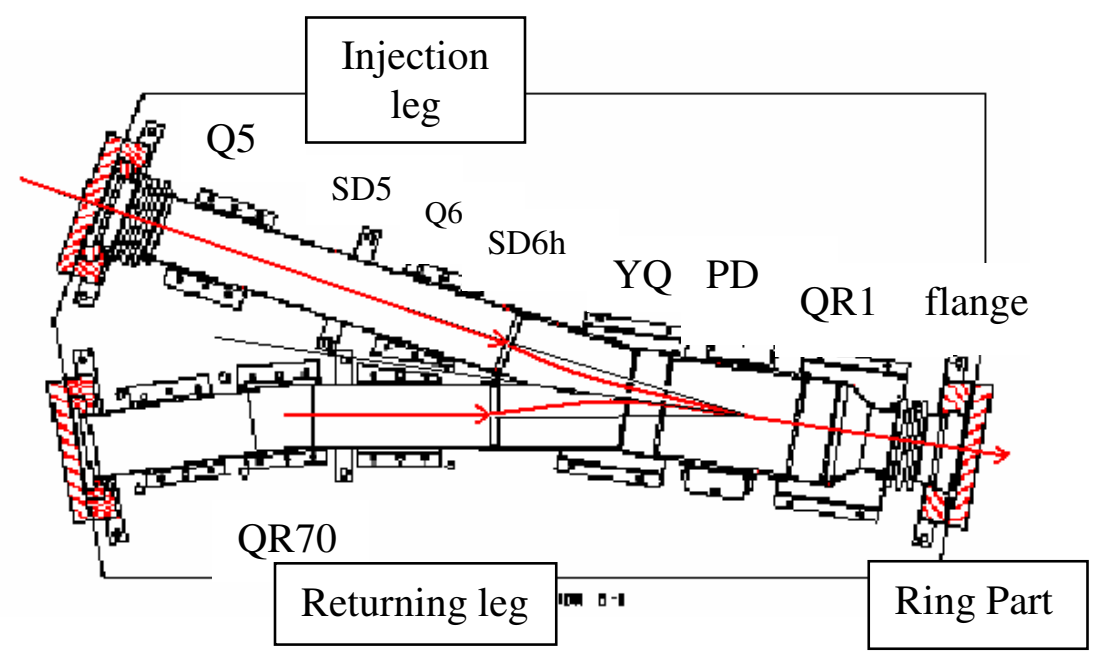

Figure 1.4 UMER Injection/recirculating section

\subsection{Organization of the thesis}

This thesis is concerned with modeling and experimental study on UMER injection/recirculating section, called Y-section. There are a total of five chapters included in this thesis. In Chapter 2, a numerical calculation of the beam centroid in an improved model is developed to decide the current settings of key magnets in the Y-section. The improved model, composed of the most important magnets of the Ysection, is reviewed and the modeling of those magnets is shown. Next, in this improved model, a numerical algorithm is implemented in the Matlab to track the beam centroid. To determine if the earth's field and dipole thickness impact the settings of key magnets, different modeling of magnetic field in the model is 
presented, including: (1) the pulsed dipole is considered as a thin dipole and does not include the earth's field; (2) the pulsed dipole is modeled as a thick dipole; (3) the earth field are added on the thick pulsed dipole model. The key magnet settings of different models mentioned above are compared to summarize the effect of the earth's field and thickness of the dipoles. Also, the solutions of magnet settings in the Y-section from previous work based on transfer matrix calculation are reviewed.

In Chapter 3, a semi-analytic numerical calculation using the single particle motion equations is developed to study the settings of the pulsed dipole for the beam injection/recirculation, the beam centroid motion and the effective lengths of overlapped magnets in the Y-section. A more realistic model is built, including injection line, injection/recirculating section and first two ring sections under the effect of the earth's field. Next, a new calculation algorithm (Matlab) based on a single particle transport idea is reported. Then the beam centroid motion after the Ysection is studied using the Matlab calculation which is single particle calculation, as well as the WARP simulation, which contains the space charge and image charge. Analysis and comparison of both simulation results are reported. Another issue of the current settings of the pulsed dipole for the beam injecting and the returning case, respectively, is followed. The assisting bending by the off-centered YQ for each case is included. The different settings for the two cases can conclude the asymmetric effect of the earth's field on the beam. Finally, WARP simulation is implemented to find more accurate effective lengths of the Y-section magnets, which are squeezed in a stringent space and form an overlapping magnetic field. 
In Chapter 4, an experiment to study the Y-section focusing on the beam centroid motion is implemented. First, the experimental setup is described including the introduction of the whole experimental system, description of characters of BPMs, the pulsed dipole and the big quadrupole in the injection/recirculating section. Secondly the settings of involved experimental devices are discussed and presented. Then the idea to obtain the beam centroid initial condition is discussed and followed by the experimental implementation details and experimental results. Next the initial beam condition is imported to both Matlab and WARP simulation to calculate the beam motions. The results from the experiment and two kinds of simulations are compared to see the space charge effect on the beam centroid motions.

Chapter 5 concludes the thesis. 


\section{Chapter 2: Improved Modeling on Y-section}

\section{$\underline{2.1 \text { Introduction of injection/recirculating section }}$}

The injection/recirculating section, called Y section, is specially designed for injecting a single long pulse into the ring and recirculating it on subsequent turns. A detailed study of this section is critical for the multi-turn operation of UMER. The typical beam operated by UMER is $100 \mathrm{~ns}$ long pulse and the circulation time around is $200 \mathrm{~ns}$, which can be easily obtained from the beam energy $10 \mathrm{keV}$ and the ring circumference $11.52 \mathrm{~m}$. The electron beam therefore occupies half of the ring. When the beam is injected fully through the pulsed dipole (see Figure 2.1), the head of the beam is already halfway around the ring. Thus the pulsed dipole must switch polarity in less than $100 \mathrm{~ns}$, to deflect the returning beam with the correct angle for recirculation. This requires a special design for the pulsed dipole. Both YQ and QR1, set on two sides of the pulsed dipole (see Figure 2.2), are effectively DC big quads, although they are pulsed for heat-management issue with a much longer pulse than that of the pulsed dipole. They are powered by same amplitude but opposite polarity according to the design requirement: YQ is horizontal defocusing quad to assist bending the beam; QR1 is horizontal focusing for the symmetry issue. Figure 2.1 shows the progress of the pulsed dipole operation [6]. 

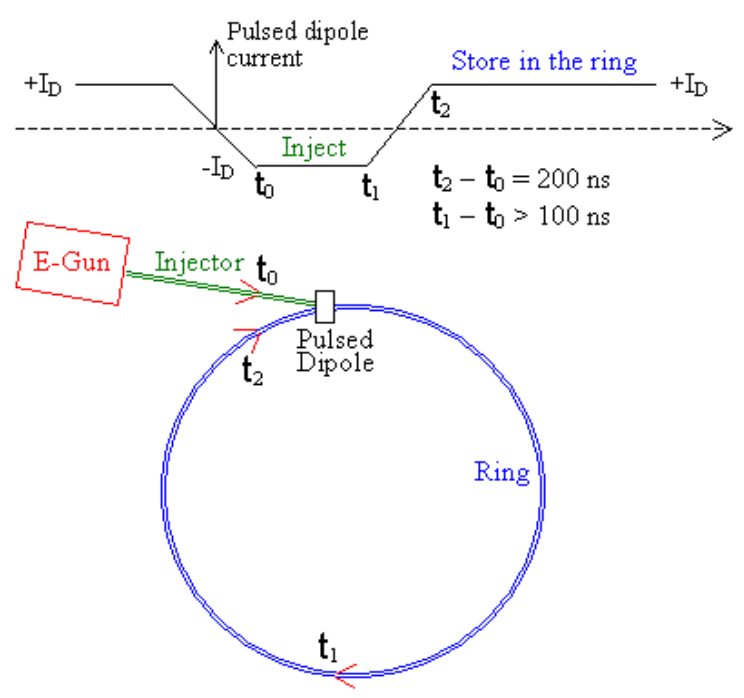

Figure 2.1 Progress of the pulsed dipole operation

The Y-section, displayed in Figure 2.2, is composed of injection line (flange upstream Q5 to $\mathrm{PD}$ ), the ring line (PD to the flange downstream $\mathrm{QR} 1$ ) and returning leg (flange upstream QR71 to PD) [22]. The injection line and returning leg are symmetric with the orbit axis of the ring line. The geometric angle between the injection line and ring line is $10^{\circ}$, while the angle for the returning part and ring part is $-10^{\circ}$. The challenge of the Y-section design is the mechanical and electrical complexity because the pulsed dipoles and big quadrupoles and other DC dipoles and quadrupoles are squeezed in a stringent space (less than $20 \mathrm{~cm}$ long) because the Ysection is actually one of UMER periodic lattices and the lattice period is $32 \mathrm{~cm}$ so quads in a periodic lattice are only $16 \mathrm{~cm}$ apart. This paucity of space and short periodic lattice require the sharing of a big magnet between the injector and the returning leg, so as to avoid using two pulsed overlapping magnets. 


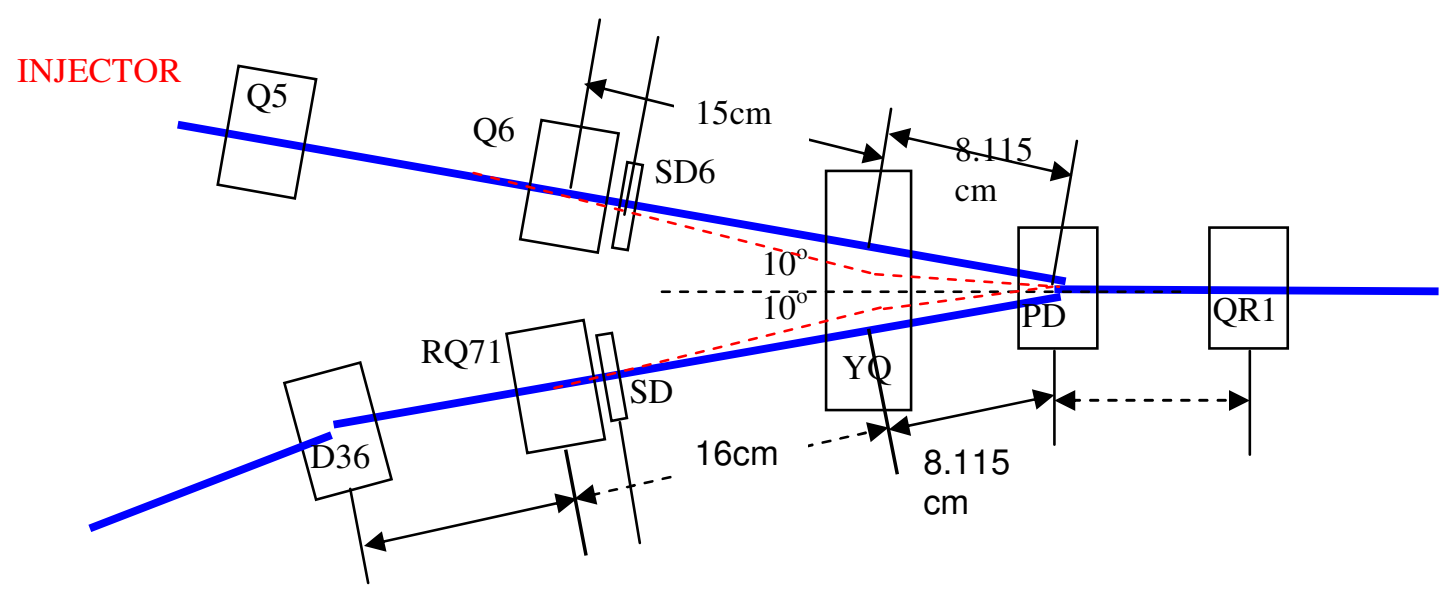

Figure 2.2 Layout of UMER injection/recirculating section

\subsection{Improved model and numerical calculations for Y-section}

\subsubsection{Key magnets' modeling in the improved model}

The most important part of the Y-section is the shared quadrupole (YQ) and the pulsed dipole (PD) because both of them are the only two magnets crossing both the injection and recirculating legs and are key magnets to deflect the beam correctly when the beam is injecting or returning into the ring. To study this key part of Ysection, we set an improved model here, composed of the big YQ, the pulsed dipole (PD) and an additional steering dipole SD6, upstream the YQ in the injection line. The improved model is shown in Figure 2.3. 


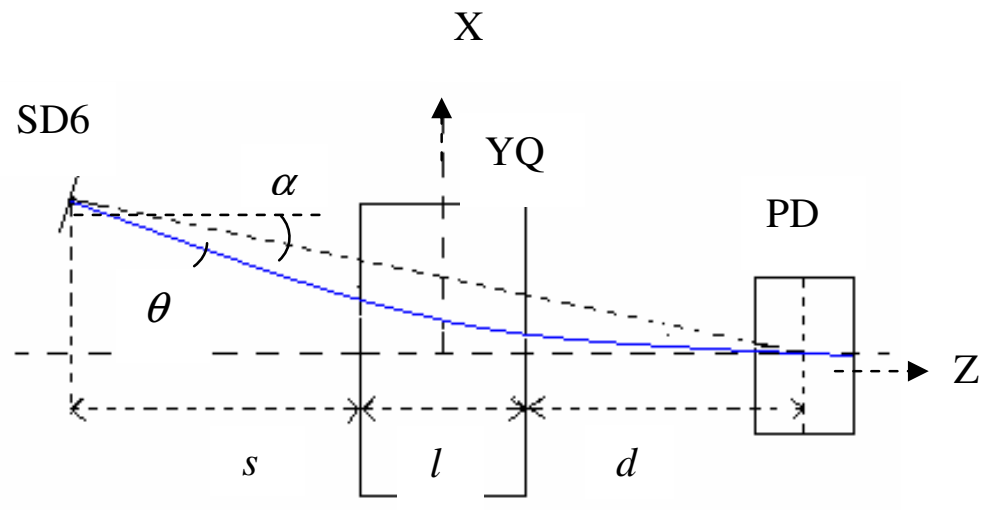

Figure 1.3 Improved model of the Y-section

For the UMER injector, $s$ is equal to $9.0 \mathrm{~cm}$ and $d$ equal to $5.31 \mathrm{~cm} ; l$ is equal to $5.38 \mathrm{~cm}$. The $\mathrm{Sd} 6$ is a typical steering dipole and modeled as a thin dipole; $\alpha=10^{\circ}$.

The YQ is a big DC magnetic quadrupole designed as defocusing in the horizontal plane and focusing vertically for electron beam. Correspondingly, the equations of motion for a single particle are

$$
\begin{aligned}
& x^{\prime \prime}+\kappa x=0 \\
& y^{\prime \prime}-\kappa y=0
\end{aligned}
$$

$\kappa$ is the quadrupole strength $\kappa=q G(z) / \gamma m \beta c$, where $G(z)$ is the quadrupole gradient; $q, m, c$ are the particle charge, mass and light speed, respectively. $\kappa(z)$ is a smooth profile with a fringe field. However, we usually use in practice an equivalent hard-edge element instead of the actual smooth profile. The hard-edge substitute has the peak value of $\kappa(z)$ and effective length, calculated by Equation (2.3).

$$
l_{\text {eff }}=\frac{1}{\kappa_{\text {peak }}} \int_{-\infty}^{+\infty} \kappa(z) d z
$$


The actual smooth profile and equivalent hard-edge approximation are calculated in Magli [23]. The results are: the effective length is $5.38 \mathrm{~cm}$; the peak gradient $G$ is equal to $1.01 \mathrm{Gauss} / \mathrm{cm} \cdot A$; the operating effective gradient is $5.33 \mathrm{Gauss} / \mathrm{cm}$ as required for having a matched beam in the ring.

In the improved model, the pulsed dipole is modeled as a wide dipole. The actual field in a wide dipole is a smooth profile of $B(z)$. Similarly, it is common to use an equivalent hard-edge magnet with effective length $l=\frac{1}{B_{\text {peak }}} \int_{-\infty}^{+\infty} B(z) d z$ to replace the actual one in calculations. The Magli is also executed to calculate the effective length and the peak field of the pulsed dipole [23]. The results are $5.18 \mathrm{~cm}$ and $0.386 \mathrm{G} / \mathrm{A}$, respectively.

\subsubsection{Numerical calculation for tracking the beam centroid in the improved model}

This Y-section model has been studied by Dr. Hui Li using beam transfer matrix methods. At that time, however, the earth's field was not accounted for in the calculation, which means the beam was considered as drifting in a straight line between magnets. Actually, since the Y-section is not be shielded by Helmholtz coils to cancel the earth's field and the typical electron beam with a very low energy, the beam centroid is definitely deflected by the earth's field, which should not be ignored. At this time, the earth's field is accounted for in our new simulation model.

Another issue worthy of mentioning is the modeling of the pulsed dipole. In the previous work, the pulsed dipole was assumed as a thin dipole. Because of its thick effective length in reality, we take the pulsed dipole as thick hard-edge dipole in 
this improved magnetic model as we mentioned above. In short, our new simulation model includes the earth's field effect and a more realistic modeling of the pulsed dipole, thus is expected to track the beam centroid in the Y-section more accurately.

With this more realistic model containing the earth's field, however, the transfer matrix in the $\mathrm{Y}$ section used before becomes much more complicated and it is difficult to calculate the beam position and relative bending angle using a simple formula as before. The approach we took instead is to develop numerical codes that simulate the beam centroid trajectory in this region.

The first thing is to build a calculation platform to calculate the beam trajectory under the improved magnetic model. Since the injection/recirculating section is designed to bend the beam in the horizontal plane, our calculation concentrates on the beam trajectory in the horizontal plane $z-x$. The steering dipole (SD6) and the pulsed dipole (PD) are designed to kick the beam in the horizontal plane, and the big defocusing YQ also helps bend the beam horizontally to left/right when beam injecting or recirculating. The strength of earth's field in our simulation model is set as 0.4 Gauss, which is the measured value of vertical component of the earth's field at the location of the Y-section.

Our idea is to track the beam centroid in a two-dimension plane $z-x$ in the magnetic field. A numerical algorithm developed in Matlab tracks the beam trajectory in the 2-D plane $z-x$, where the positive $z$ is the beam propagation direction after the pulsed dipole, and the positive $x$ is perpendicular to $z$ in the $z-x$ plane outwards the ring. Note that the beam motion in the $z-x$ plane is affected greatly by the vertical component of the earth's field, which is approximate 0.4 Gauss. We choose 
the origin in our simulation to be the center of the big quadrupole (YQ). To track the beam centroid, we ignore image charge so the centroid becomes a single particle, which follows the force law in the 2-D plane $z-x$, shown as Equation (2.4) and (2.5).

$$
\begin{aligned}
& \ddot{x}=\frac{d v_{x}}{d t}=\frac{F_{x}}{m} \\
& v_{z}=v_{z 0}
\end{aligned}
$$

$v_{x}$ is the beam velocity $\mathrm{X}$ component in the horizontal plane; $m$ is electron mass; $F_{x}$ is the $\mathrm{X}$ component of the Lorentz force on the particle; $v_{z}$ is the beam velocity $\mathrm{Z}$ component, assumed as a constant along the beam transport because it is much larger than the $\mathrm{X}$ component $v_{x}$. Thus the Lorentz force $\mathrm{Z}$ component due to $v_{x}$ is so small and can be ignored. Therefore, $v_{z}$ is kept as same as $v_{z 0}$, the initial $\mathrm{Z}$ component beam velocity. The initial beam velocity components are $v_{x 0}$ and $v_{z 0}$ in the following equations.

$$
\begin{aligned}
& v_{x 0}=v \cdot \sin (\theta) \\
& v_{z 0}=v \cdot \cos (\theta)
\end{aligned}
$$

$v$ is the beam initial velocity, which can be calculated by the beam energy of $10 \mathrm{keV} . \theta$ is the beam centroid angle out of SD6.

Equation (2.8) is derived from Equation (2.4); $F_{x}$ can be calculated from Equation (2.9). $\mathrm{q}$ is the electron charge; $B_{y}$ is the total magnetic field at one point vertically; $v_{z}$ is constant as we mentioned.

$$
d v_{x}=\frac{F_{x}}{m} d t
$$




$$
\begin{aligned}
& F_{x}=q v_{z} B_{y} \\
& d v_{x}=\frac{q v_{z} B_{y}}{m} d t
\end{aligned}
$$

The beam centroid numerical calculation in the Matlab code is based on the leap-frog iteration. The Equation (2.10) is used to derive the relation of the (i+1)thstep beam $\mathrm{X}$ velocity component and the (i)th-step $\mathrm{X}$ velocity. Because one step is tiny, we have the following equations derived from Equation (2.10).

$$
\begin{aligned}
& v_{x, i+1}-v_{x, i}=\frac{q v_{z} B_{y}}{m} d t \\
& v_{x, i+1}=v_{x, i}+\frac{q v_{z} B_{y}}{m} d t
\end{aligned}
$$

With this equation, $v_{x}$ at the step $(i+1)$ can be calculated by the adjacent step ( $i$ ). This equation is a basic equation for leap-frog method.

From leap-frog idea, the $v_{x}$ is kept unchanged in one tiny step thus the beam position at one point $\left(x_{i+1.5}, z_{i+1.5}\right)$ can be calculated by the adjacent upstream point $\left(x_{i+0.5}, z_{i+0.5}\right)$ through Equation (2.13) and (2.14). Beginning at the known beam initial position and velocity, the beam centroid positions in the $z-x$ plane can be calculated by the leap-frog iteration.

$$
\begin{aligned}
& x_{i+1.5}=x_{i+0.5}+v_{x, i+1} \cdot d t \\
& z_{i+1.5}=z_{i+0.5}+v_{z} \cdot d t
\end{aligned}
$$

With the above beam centroid numerical algorithm, the beam centroid trajectory can be found in a given field with specific initial beam conditions. 
Next, a Matlab algorithm is developed to study the improved model magnets and calculate the settings of the magnets by tracking the beam centroid trajectory in the model. According to UMER design, the beam centroid is required to pass through the center of the pulsed dipole with a zero exit angle at the right edge of the pulsed dipole. To satisfy these two variable requirements, we need two free parameters from the improved model. Our algorithm is to successively iterate the bending angle of SD6 and the magnetic field strength of the pulsed dipole to make both the centroid X position and its exit angle out of the pulsed dipole zero at the right edge of the pulsed dipole. In the calculation, we assume the beam enters SD6 perfectly aligned, meaning that the SD6 bending angle is the exit angle from SD6, i.e. $\theta$.

\subsubsection{Comparison of key magnet settings calculated from the improved model to}

\section{previous work}

At first, we compare the results of our Matlab calculation with the previous work done by Dr. Hui Li. In that model, the pulsed dipole was assumed as a thin dipole and the earth's field is not accounted for; the calculation is based on single particle transfer matrices of the Y-section. Please refer to [21] for details of the previous calculation. To compare with previous work, we firstly consider the improved model with the exactly same conditions as the previous calculation: we do not include effect of the earth's field and assume the pulsed dipole as a thin lens, locating at the midpoint of the wide pulsed dipole. We call this modified model as Model 1. 
First we do the previous calculation and the results of bending angles of SD6 and the pulsed dipole are shown in Table 2.1. Second, the beam centroid is tracked in Model 1 using the Matlab algorithm. The beam centroid is shown in Figure 2.4, in which the beam centroid passes through $X=0$ offset at $Z=8$ where the thin pulsed dipole is located. The calculation result is that when the SD6 bends the beam with an angle of $2.156^{\circ}$, which sends the beam centroid to the center of the pulsed dipole, therefore the beam should be deflected by the pulsed dipole with $6.983^{\circ}$ to exit with zero angle.

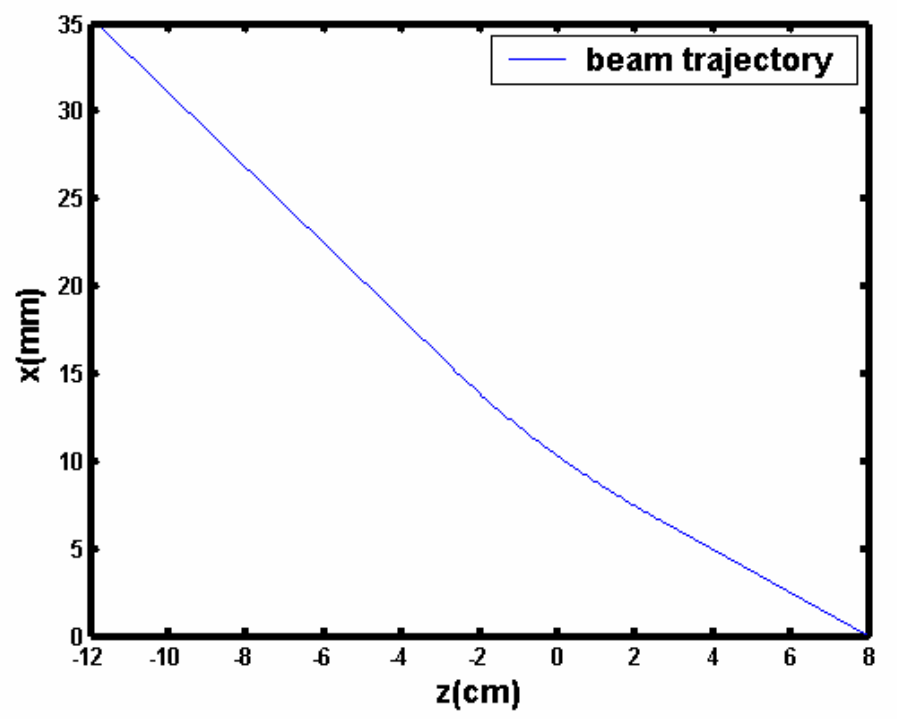

Figure 2.4 Beam trajectory in Model 1: with thin PD/without earth field

The comparison of the results from the previous calculation and Model 1 is shown in Table 2.1, demonstrating that the new calculation results agree well with the previous calculation based on transfer matrix. 
Table 2.1 Comparison of Model 1 calculation results to previous work

\begin{tabular}{|c|c|c|}
\hline & SD6 angle & PD angle \\
\hline Previous work & $2.08^{\circ}$ & $7.04^{\circ}$ \\
\hline \hline Model 1 & $2.156^{\circ}$ & $6.983^{\circ}$ \\
\hline
\end{tabular}

\subsubsection{Beam trajectories and key magnet settings with different modeling for the pulsed dipole and earth's field}

We divide the calculation into two models according to different consideration of the pulsed dipole and the earth's field so that the effects of the pulsed dipole thickness and the earth's field on the beam trajectory can be indicated and compared clearly.

First, we consider the pulsed dipole as a thick lens with $5.18 \mathrm{~cm}$ effective length; the earth's field is not accounted for at this time, either. This is called Model 2. We need to scan the bending angle of SD6 to find the exact SD6 bending angle and magnetic strength of the pulsed dipole that makes the beam exit from the right edge of PD with zero x-offset and divergence.

We develop two procedures (A and B) to find suitable SD6 bending angle and bending angle of the pulsed dipole, and correspondingly, the magnetic field, in Model 2. In procedure A, we scan the SD6 bending angle around the value of $2.156^{\circ}$, which is the suitable SD6 bending angle when the pulsed dipole as thin dipole, and the PD strength, find that when the PD strength is 7.7 Gauss, i.e. $6.998^{\circ}$ and SD6 bends the beam by $2.155^{\circ}$, the beam centroid passes through the zero $\mathrm{X}$ offset at the right edge of PD with zero angle. In procedure B, we set the SD6 bending angle equal to $2.155^{\circ}$, 
and then scan the magnetic field strength of the pulsed dipole. The result is that when the PD strength is 7.7 Gauss, the beam hits the expected location. The result obtained from procedure B agrees with procedure A very well. The beam centroid trajectory in Model 2 is shown in Figure 2.5.

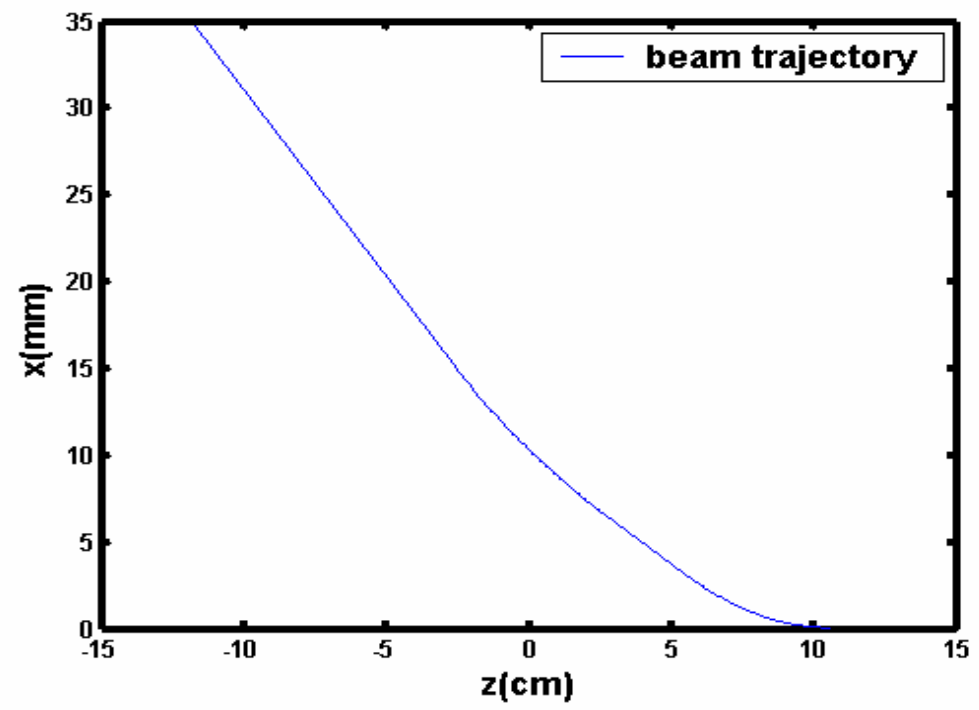

Figure 2.5 Beam trajectory in Model 2: with thick PD/without earth field

Second, to assess the effect of the earth's field, we add a uniform field over the entire area equal to 0.4 Gauss onto Model 2, which is called Model 3. We first set the bending angle of SD6 equal to $2.155^{\circ}$ and scan the pulsed dipole strength, but at this time, in Model 3, we can not find a suitable magnetic strength of the pulsed dipole to make the beam transport as desired. This predicts that the earth's field impacts the beam trajectory.

To find the bending angle of SD6 and the PD strength in this model containing the earth's field, we improve the two iterative procedures (A and B) for Model 3. In procedure A, we scan both the bending angle of SD6 around the value of 
$2.0^{\circ}$ and the PD strength, until the beam is centered at the PD right edge with zero exit angle. The result from the simulation is that the SD6 angle is $1.585^{\circ}$, and the PD strength 8.5 Gauss, i.e. the bending angle of the $\mathrm{PD} 7.153^{\circ}$. In procedure $\mathrm{B}$, we set the SD6 bending angle equal to $1.585^{\circ}$, and then scan the magnetic field strength of the pulsed dipole. The result is that when the PD strength is 8.5 Gauss, the beam hits the expected location and exit with zero divergence. The result obtained from procedure B agrees well with procedure A well again fro Model 3; the beam centroid in Model 3 is shown in Figure 2.6.

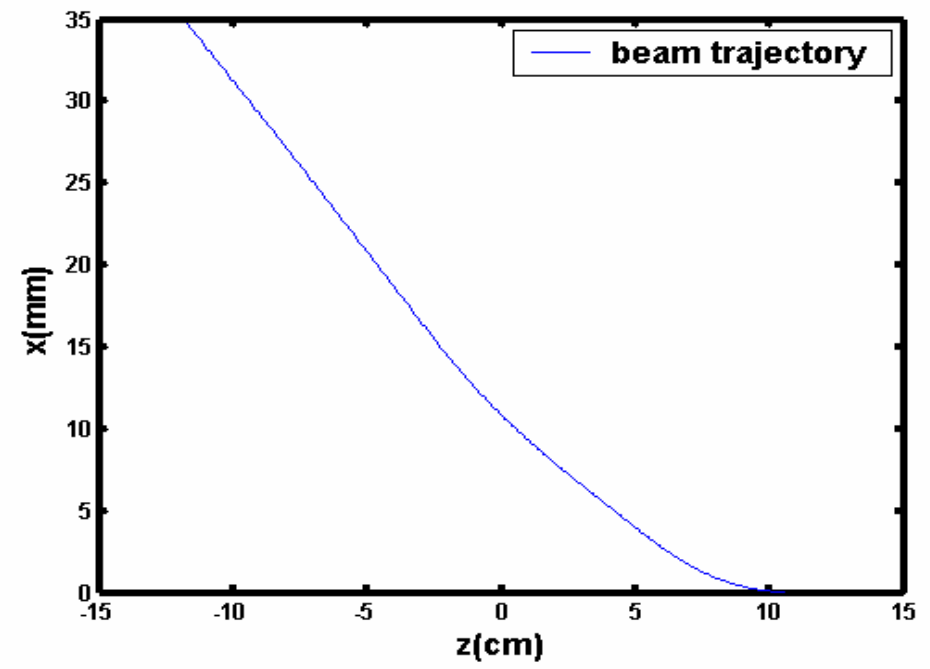

Figure 2.6 Beam trajectory in Model 3: with thick PD \& with earth field

Usually the beam trajectory is described by the coordinates in the laboratory frame, called $\left(x_{l}, z_{l}\right)$, as we have done in the $2 \mathrm{D} \mathrm{x}-\mathrm{z}$ system. The defect of describing beam positions in the laboratory frame is that it is not displaying the trajectory difference explicitly, such as in Figure 2.5 and 2.6, it is difficult to observe the difference of the beam trajectory for the two magnetic models. But the difference is 
what we are really interested in. To compare the results of the wide PD model with/without the earth field, we develop a new program that tracks the beam in a new reference, called WARP reference, which is composed of the line before YQ (Region I) and the arc in YQ through the pulsed dipole (Region II). The coordinates of beam position in the WARP reference are $\left(x_{w}, z_{w}\right)$, which can be transformed from the laboratory coordinates, as in the following equations. The coordinate $z_{w}$ represents the direction along the pipe center; the $x_{w}$ represents the beam centroid deviation from the pipe center perpendicular to the pipe center in the horizontal plane.

We define $s_{1}=\frac{D}{\cos \alpha}-l_{p d} / 2$ and $s_{2}=s_{1}+R \cdot \alpha . \quad l_{p d}$ is the effective length of the pulsed dipole, equal to $5.18 \mathrm{~cm}$ in the case of UMER; $D=d+l / 2=8 \mathrm{~cm}, l$ is YQ effective length of $5.38 \mathrm{~cm} ; \mathrm{d}=5.31 \mathrm{~cm}$ and $\alpha=10^{\circ} ; R=\frac{l_{p d} \cdot \sin \alpha}{2(1-\cos \alpha)}$.

Equation (2.15) and (2.16) are the coordinate transformations in Region I.

$$
\begin{aligned}
& x_{w}=x_{l} \cdot \cos \alpha+z_{l} \cdot \sin \alpha-D \cdot \sin \alpha \\
& z_{w}=z_{l} \cdot \cos \alpha-x_{l} \cdot \sin \alpha+D \sin ^{2} \alpha / \cos \alpha
\end{aligned}
$$

In Region II, the following two equations are applied to convert the coordinates of the specific beam position.

$$
\begin{aligned}
& z_{w}=s_{2}-R \cdot \operatorname{actag}\left(\frac{z_{l}-D-l_{p d} / 2}{x_{l}-R}\right) \\
& x_{w}=R+\left(x_{l}-R\right) \sqrt{1+\left(\frac{z_{l}-D-l_{p d} / 2}{x_{l}-R}\right)^{2}}
\end{aligned}
$$


The beam horizontal trajectories in the WARP reference are shown in Figure 2.7. From the figure, we can clearly see that the effect of the earth's field is significant on the beam trajectory, which can result in serious mis-steering if not properly accounted for. In this figure, the zero of the vertical axis is pipe center.

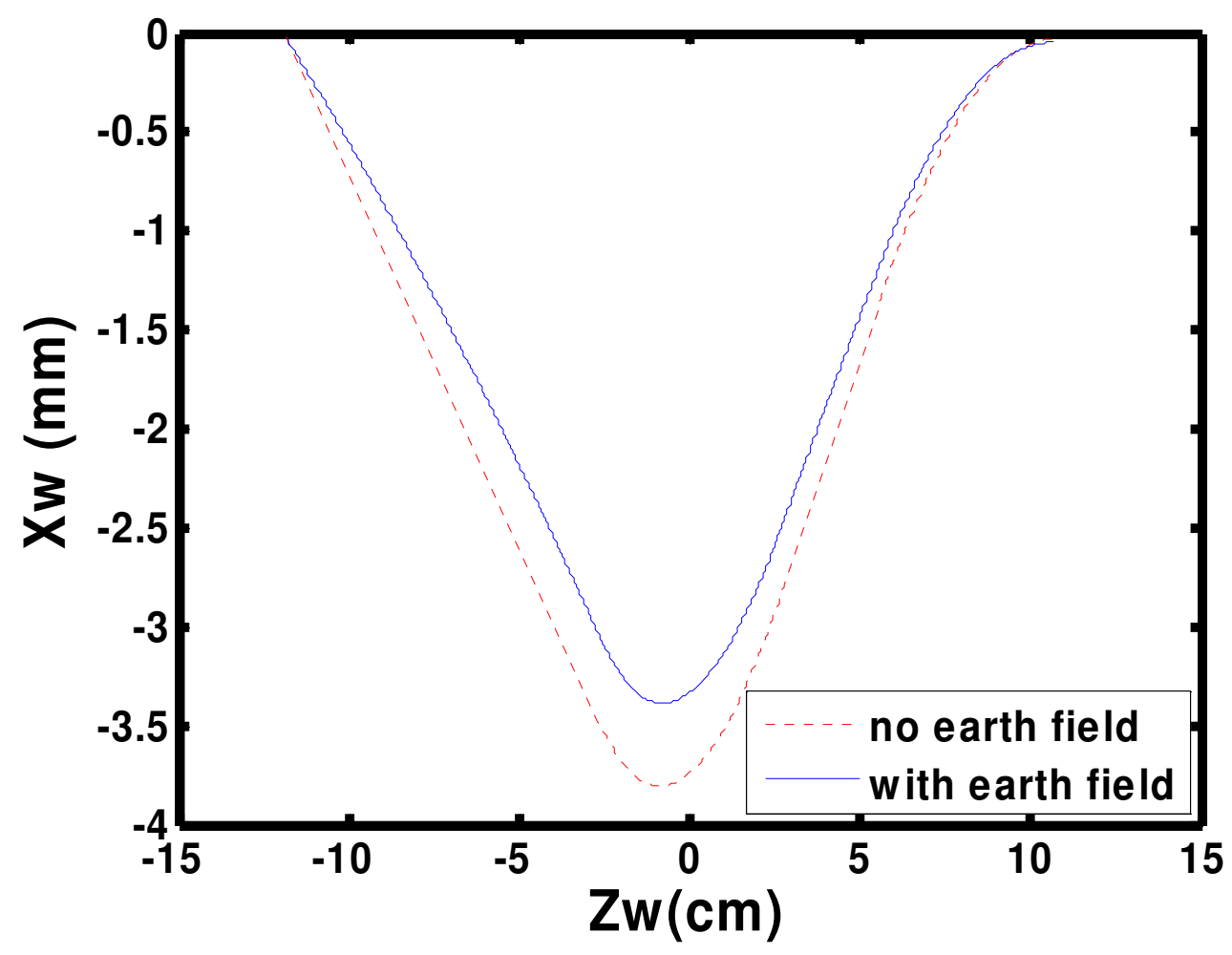

Figure 2.7 Comparison of beam centroid trajectories in WARP frame: with thick PD, with/without Earth field

This result shown in the above figure shows a good agreement with the beam centroid trajectory in the previous WARP simulation, shown in Figure 2.8 [6]. In the WARP simulation without considering the earth's field, the beam centroid deflects from the pipe center to around $-3.8 \mathrm{~mm}$ at the location of $\mathrm{YQ}$ in the $\mathrm{X}$ direction when beam injects, which shows a good agreement with the beam centroid trajectory shown in Figure 2.7, represented by the red dash-line, which is beam centroid without 
considering the earth's field, deflecting also around $-3.8 \mathrm{~mm}$ from the pipe center in the horizontal plane.
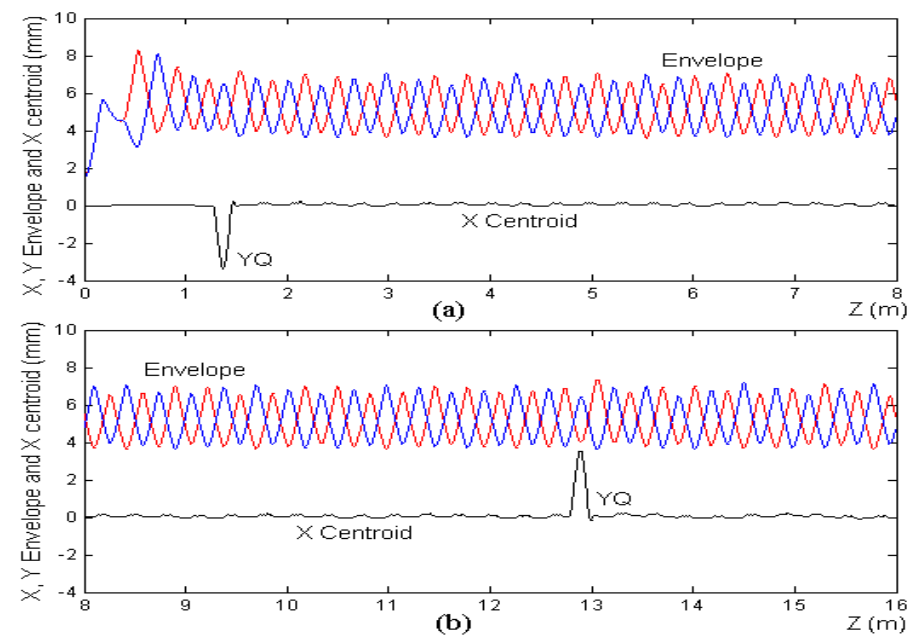

Figure 2.8 Beam X centroid and envelopes for one turn in WARP simulation

\subsection{Comparison of magnets' settings in the improved model with different modeling of the PD thickness and the earth's field}

Table 2.2 compares the Matlab calculation results of different modeling of the pulsed dipole and the earth's field on the Y-section, as well as corresponding current settings and magnetic field strengths.

In Table 2.2, $\theta$ is bending angles by SD6 and $\theta^{\prime}$ is bending angles of the pulsed dipole from the simulation of different models. The corresponding operation currents are calculated by Equation (2.19). SD6 integral field is $3.627 \mathrm{G} \cdot \mathrm{cm} / \mathrm{A}$, peak field is $1.18 \mathrm{G} / \mathrm{A}$; PD integral field is $2 \mathrm{G} \cdot \mathrm{cm} / \mathrm{A}$ and peak field is $0.386 \mathrm{G} / \mathrm{A}$. The magnetic strengths of dipoles can be calculated by Equation (2.20). T-PD is thick PD; Be is the earth's field; $S$ is magnet strength of the pulsed dipole directly from the simulation; $S^{\prime}$ is the PD strength calculated through Equation (2.20), which agree well with the results obtained from the simulation S. 


$$
\begin{aligned}
& I=\frac{\theta \cdot \gamma \cdot m \cdot \beta \cdot c}{q \cdot \text { Int.Field }} \\
& \text { Dipole.strength }=\text { Peak.field } * I
\end{aligned}
$$

Table 2.2 Comparison of magnet settings in the improved model on different modeling of the pulsed dipole and earth's field

\begin{tabular}{|c|c|c|c|c|c|c|c|}
\hline & \multicolumn{3}{|c|}{ SD6 } & \multicolumn{4}{c|}{ Pulsed Dipole (PD) } \\
\cline { 2 - 8 } & $\begin{array}{c}\theta \\
(\mathrm{deg})\end{array}$ & $\begin{array}{c}\text { Current } \\
(\mathrm{A})\end{array}$ & $\begin{array}{c}\text { Strength } \\
(\mathrm{G})\end{array}$ & $\begin{array}{c}\theta^{\prime} \\
(\mathrm{deg})\end{array}$ & $\begin{array}{c}\text { Current } \\
(\mathrm{A})\end{array}$ & $\begin{array}{c}\mathrm{S} \\
(\mathrm{G})\end{array}$ & $\begin{array}{c}\mathrm{S}^{\prime} \\
(\mathrm{G})\end{array}$ \\
\hline Thin PD & 2.156 & 3.519 & 4.152 & 6.983 & 20.671 & - & 8.0 \\
\hline T-PD & 2.155 & 3.518 & 4.151 & 6.998 & 20.716 & 7.7 & 8.0 \\
\hline T-PD + Be & 1.585 & 2.587 & 3.053 & 7.153 & 21.175 & 8.5 & 8.2 \\
\hline
\end{tabular}

We compare the thin PD case with the wide PD case, to determine if the thickness of the dipole affects the solution. As shown in Table 2.2, there are hardly any differences for the bending angles of the SD6 and the PD, as well as current settings and dipole strengths, meaning we can safely assume a thin PD in the calculations without affecting the results. Similarly, the thick PD case and the case of thick PD plus the earth's field are compared to see if the earth's field affects the solution. As shown in Figure 2.7 and Table 2.2, the beam centroid trajectories for the two cases are different and there are also differences between the magnet settings. When the earth's field is accounted for, the SD6 strength is reduced while the PD magnetic strength increases, compared with the model without the earth's field. Conclusion can be made that the earth's field can not be ignored in the calculation. 


\section{Chapter 3 More Accurate Modeling and Calculations on Y-}

\section{Section}

In this chapter, we introduce a more realistic magnetic model originating from IC2 and extending to RC2. A new approach to track the beam centroid in the horizontal plane based on single particle transport equations within this model will be studied. Also, the beam centroid motion will be calculated using both the above new approach in Matlab and the WARP model containing the space charge. The results are analyzed and compared. Some issues such as the pulsed dipole settings for injection/recirculation and the calculation of overlapped fields are discussed.

\subsection{More accurate and extended magnetic modeling from IC2 to RC2}

\subsubsection{Layout of the extended magnetic model}

Although our most interesting stuff is the Y section, it is still important to watch beam centroid motions after the $\mathrm{Y}$ section in the ring; also, the $\mathrm{Y}$ section in UMER machine is a black box without diagnostics and the beam can only be monitored at IC2 upstream or RC1 and RC2 downstream. Therefore in order to see the simulation results in the future experiment, it is necessary to extend the magnetic modeling, which originates from IC2 in the injection line and passes through RC1 until RC2. The extended magnetic model for the beam centroid calculation is shown in Figure 3.1. 


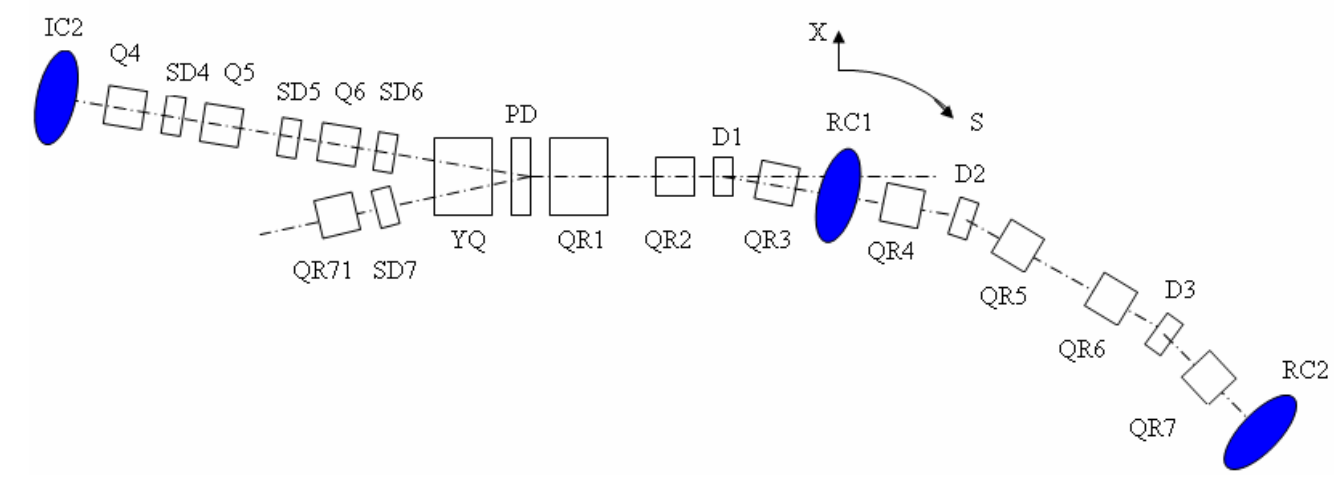

Figure 3.1 Layout of more accurate and extended model for Y-section

The magnetic model is built in the horizontal plane including the injection line, the Y-section and followed by the first three periodic lattices on the ring circle. The injection line and the ring circle meet at the center of the pulsed dipole with a $10^{\circ}$ difference, as shown in Figure 3.1. The ring circle is approximately described as four single lines in the order of PD-D1, D1-D2, D2-D3 and D3-RC2, as shown in Figure 3.1. For example, the line of D1-D2 meets the line of PD-D1 at the center of D1, and rotates $10^{\circ}$ clockwise with the crossing point, because according to UMER design one lattice including one ring dipole (D1), two different polarity quadrupoles (QR2 and QR3) separated by the dipole and four equal long drift space, is distributed along a $10^{\circ}$ arc in the whole ring circle.

The layout details of the magnets on the model are measured directly from UMER machine, shown in Table 3.1.

Table 3.1 Layout details of magnets in the extended model of Y-section

\begin{tabular}{cccccccc}
\hline IC2-Q4 & Q4-SD4 & Q4-Q5 & Q5-SD5 & Q5-Q6 & Q6-SD6 & IC2-PD & SD7-PD \\
$9.9 \mathrm{~cm}$ & $4.8 \mathrm{~cm}$ & $14.5 \mathrm{~cm}$ & $6.1 \mathrm{~cm}$ & $16 \mathrm{~cm}$ & $3.75 \mathrm{~cm}$ & $64.2 \mathrm{~cm}$ & $20 \mathrm{~cm}$ \\
& & & & & & & \\
\hline
\end{tabular}


The section from the YQ to RC2 is composed of four UMER periodic lattices. Basically, the magnets in the Y-section, i.e. YQ, PD and QR1, and the drift spaces there form the first lattice in the ring. Please refer to Chapter 1 for details of lattice layout.

\subsubsection{Modeling of key magnets in the more accurate and extended magnetic}

\section{model}

The modeling of the Y-section magnets has been discussed in Chapter 2. The big quad YQ is modeled as a hard-edge horizontal defocusing quadrupole and QR1 is described symmetrically but focusing beam horizontally. The effective length for both YQ and QR1 in the model is $5.21 \mathrm{~cm}$, not $5.38 \mathrm{~cm}$ in Chapter 2, because several magnets residing in a stringent space at this time result in an overlapped magnetic field and change effective lengths of magnets. The overlapped magnetic fields in the Y-section will be studied later in this section. The peak gradient of YQ and QR1 should be specified. The pulsed dipole is considered as a thin dipole from the conclusion of Chapter 2.

According to UMER design, Q5 and Q6 are the third generation [22], with effective length of $3.72 \mathrm{~cm}$ and operation current is -2.11 A for horizontal defocusing Q5 and 2.11 A for Q6 which focusing beam in the horizontal plane. The thick quadrupole transfer matrix for beam motions is used to in the model to calculate the beam position and angle after a quad $\left(x, x^{\prime}\right)$ according to the beam position and angle just before the quad $\left(x_{0}, x_{0}{ }^{\prime}\right)$. The transfer matrix is at the followings. 


$$
\begin{aligned}
& \left(\begin{array}{l}
x \\
x^{\prime}
\end{array}\right)=\left(\begin{array}{cc}
\cosh \sqrt{\kappa_{Q 5} l} & \frac{1}{\sqrt{\kappa_{Q 5}}} \sinh \sqrt{\kappa_{Q 5} l} \\
\sqrt{\kappa_{Q 5}} \sinh \sqrt{\kappa_{Q 5}} l & \cosh \sqrt{\kappa_{Q 5}} l
\end{array}\right)\left(\begin{array}{l}
x_{0} \\
x_{0}{ }^{\prime}
\end{array}\right) \\
& \left(\begin{array}{l}
x \\
x^{\prime}
\end{array}\right)=\left(\begin{array}{cc}
\cos \sqrt{\kappa_{Q 6}} l & \frac{1}{\sqrt{\kappa_{Q 6}}} \sin \sqrt{\kappa_{Q 6} l} l \\
-\sqrt{\kappa_{Q 6}} \sin \sqrt{\kappa_{Q 6}} l & \cos \sqrt{\kappa_{Q 6} l}
\end{array}\right)\left(\begin{array}{l}
x_{0} \\
x_{0}{ }^{\prime}
\end{array}\right)
\end{aligned}
$$

Equation (3.1a) is for Q5, $l$ is effective length of $3.72 \mathrm{~cm} ; \kappa_{Q 5}$ is calculated by

$$
\kappa=\frac{q \cdot \text { Gradient }_{\text {peak }} \cdot I_{\text {operation }}}{\gamma \cdot m \cdot \beta \cdot c}
$$

$q$ is electron charge; $\gamma, \beta$ are decided by $10 \mathrm{keV}$ beam energy; $m$ is electron mass and $c$ is light speed; for Q5, the Gradient $t_{\text {peak }}$ is equal to $3.61 \mathrm{G} / \mathrm{cm} / \mathrm{A}, I_{\text {operation }}=$ -2.11 A so that $\kappa_{Q 5}=-224.47 m^{-2}$. The negative sign means that horizontal defocusing. For Q6, Gradient ${ }_{\text {peak }}$ is also $3.61 \mathrm{G} / \mathrm{cm} / \mathrm{A}$, and $I_{\text {operation }}$ is $2.11 \mathrm{~A}$ therefore $\kappa_{Q 6}$ is $224.47 \mathrm{~m}^{-2}$ for horizontal focusing.

In the magnetic model, the ring quadrupoles (QR2 to $Q R 7)$ are set horizontally defocusing, focusing in turn. That means QR2 is horizontal defocusing and QR3 is focusing horizontally. All the ring quadrupoles are the second generation quads [22], and operation current is $\pm 1.88 \mathrm{~A}$; the positive sign is for horizontal focusing quads while the negative sign is for quads defocusing horizontally. All the ring quads are modeled as thin quadrupole here and the transfer matrix for thin quads are used in the model to describe the effect of the ring quad on beam centroid, shown in Equation (3.3). 


$$
\left(\begin{array}{l}
x_{q} \\
x_{q}{ }^{\prime}
\end{array}\right)=\left(\begin{array}{cc}
1 & 0 \\
-1 / f_{q} & 1
\end{array}\right)\left(\begin{array}{l}
x_{q 0} \\
x_{q 0}{ }^{\prime}
\end{array}\right)
$$

$\left(x_{q 0}, x_{q 0}{ }^{\prime}\right)$ are beam centroid position and angle before the quadrupole and $\left(x_{q}, x_{q}{ }^{\prime}\right)$ is that of beam centroid after the quad. $f_{q}$ is focal length of the quadrupole calculated by $f_{q}=\frac{1}{\kappa_{q} \cdot l_{q}} \cdot l_{q}$ is effective length for the ring quads, equal to $3.63 \mathrm{~cm}$ for all; $\kappa_{q}$ can be calculated by Equation (3.2), Gradient ${ }_{p e a k}$ for the ring quad is 4.14 $\mathrm{G} / \mathrm{cm} / \mathrm{A}$ and $I_{\text {operation }}$ is -1.88 A for horizontal defocusing quads and $1.88 \mathrm{~A}$ for horizontal focusing quads. The result is that for horizontal defocusing quads QR2, QR4 and QR6, $f_{q}=-0.1197 \mathrm{~m}$; for horizontal focusing quads QR3, QR5 and QR7, $f_{q}$ is equal to $0.1197 \mathrm{~m}$.

All the dipoles involved including steering dipoles on the injection (i.e. SD4h, SD5h, SD6h, h means horizontal) and ring dipoles in the first three lattices (i.e. D1, D2, D3) are modeled as thin dipoles. The beam centroid is only kicked by some angle by the thin dipole but the position is not deflected by the thin dipole. The following equation describes thin dipole effect on beam centroid.

$$
\left(\begin{array}{l}
x_{d} \\
x_{d}{ }^{\prime}
\end{array}\right)=\left(\begin{array}{ll}
1 & 0 \\
0 & 1
\end{array}\right)\left(\begin{array}{l}
x_{d 0} \\
x_{d 0}{ }^{\prime}
\end{array}\right)+\left(\begin{array}{l}
0 \\
\theta_{d}
\end{array}\right)
$$

$\left(x_{d 0}, x_{d 0}{ }^{\prime}\right)$ is beam centroid position and angle before the thin dipole and $\left(x_{d}, x_{d}{ }^{\prime}\right)$ is that of beam centroid after the thin dipole. $\theta_{d}$ is the bending angle by the thin dipole. 
The earth's field is accounted for along the whole model except the section from IC2-SD4 because a Helmholtz coil is used there to cancel the earth's field. The earth's field on the rest part varies at different sections. Table 3.2 shows the vertical earth's field amplitudes which are used in the simulation at different places in the model.

Table 3.2 Earth's fields at different locations along the model

\begin{tabular}{ccrrr}
\hline Injection line (SD4-SD6) & PD location & D1 location & D2 location & D3 location \\
$400 \mathrm{mG}$ & $407 \mathrm{mG}$ & $377 \mathrm{mG}$ & $373 \mathrm{mG}$ & $357 \mathrm{mG}$ \\
\hline
\end{tabular}

\subsubsection{Calculation of more accurate effective lengths of big magnets in the} overlapped field at Y-section

In our more realistic simulation model, shown in Figure 3.1, the steering dipoles (SD5h and SD6h) are modeled as thin dipoles while the big quadrupoles (YQ and QR1) are considered as long, hard-edge magnets. The problem is that YQ and QR1 are modeled separately in the Matlab simulation, however, in reality, since these magnets are located in a tight space, the magnetic fields of the YQ, PD and QR1 are overlapped, as shown in Figure 3.2. Thus it is necessary to calculate the overlapped field, so as to obtain more accurate effective lengths of YQ and PD for the Matlab model. 


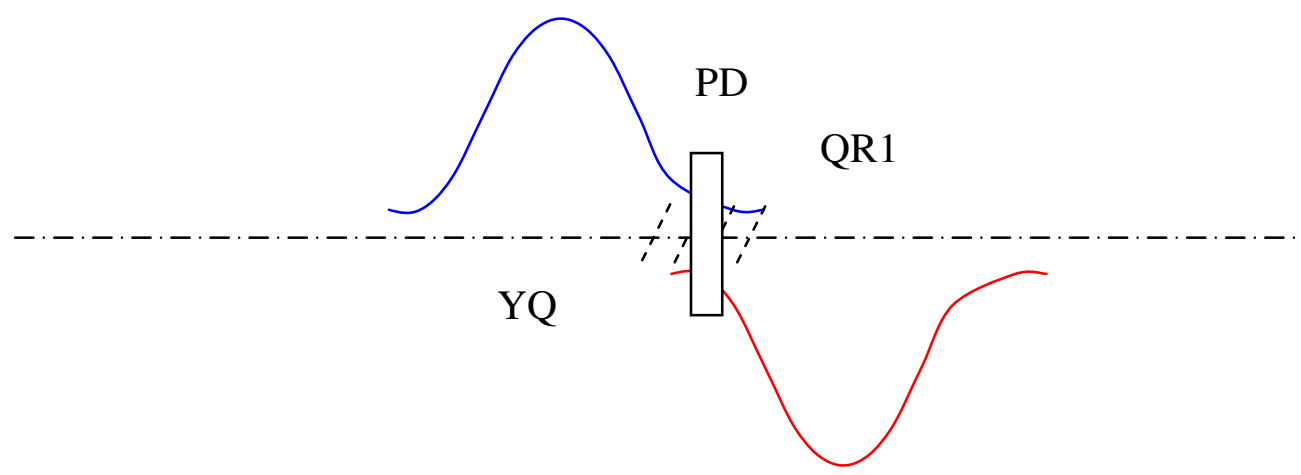

Figure 3.2 Overlapped magnetic fields of big magnets in the restricted Y region

The WARP code is implemented to calculate the overlapped magnetic field [24]. The WARP models the complicated overlapping magnetic fields of the thick magnets close to each other, and determines the more realistic effective lengths of YQ and pulsed dipole in the overlapping field. Combined with Magli calculation of the magnetic field at a specific point, the magnetic field along the pipe center in the $\mathrm{Y}$ region is calculated and plotted, shown in Figure 3.3. S is the pipe orbit. Since the YQ is off-center from the pipe orbit, it kicks the beam centroid as a dipole when beam passes. In the figure, the field on the pipe orbit in YQ has same polarity, smaller amplitude with PD, which will helps the PD bend the beam. 


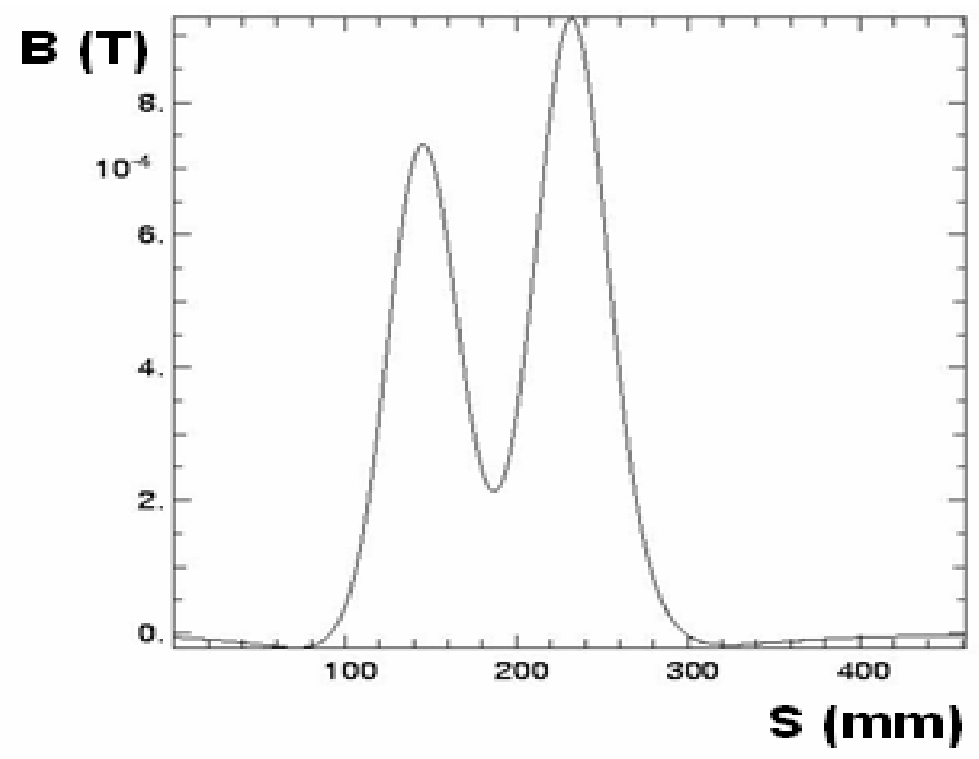

Figure 3.3 Magnetic fields on the pipe center in the Y-section involved with overlapped magnets

A numerical integration is executed to obtain the more accurate effective lengths for involved magnets. The more accurate results are compared with the effective lengths calculated from singly considering every magnet, shown in Table 3.3. The table shows that the effective lengths for $Y Q$ and QR1 are $5.21 \mathrm{~cm}$; the effective length of the pulsed dipole is $4.90 \mathrm{~cm}$. This result has been used in the previous Matlab calculations to make the calculation more accurate.

Table 3.3 More accurate effective lengths of magnets overlapped in Y-section

\begin{tabular}{|c|c|c|c|}
\hline & YQ $(\mathrm{cm})$ & QR1 $(\mathrm{cm})$ & PD $(\mathrm{cm})$ \\
\hline Overlap & 5.21 & 5.21 & 4.90 \\
\hline Singly & 5.38 & 5.38 & 5.18 \\
\hline
\end{tabular}


With the more accurate Y-section model, the beam centroid passing through this complicated region is tracked and shown in Figure 3.4.

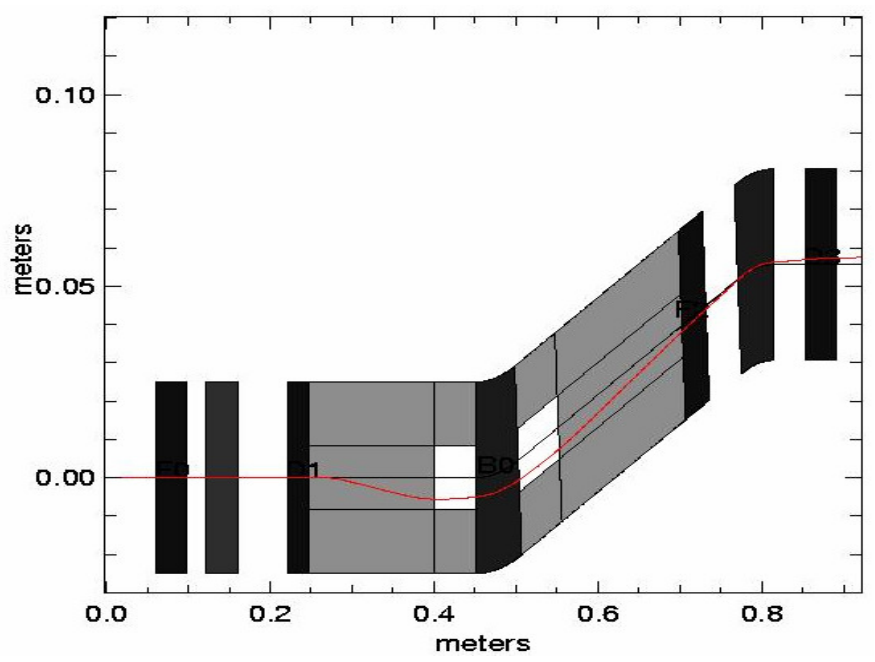

Figure 3.4 Beam centroid in the more realistic Y-section model with WARP simulation

\subsection{Numerical calculation of beam centroid with Matlab in the more}

\section{accurate model}

\subsubsection{Leap-frog iteration method for beam centroid calculations}

We use a new idea to develop the simulation algorithm for tracking beam centroid trajectory in above calculation model. The traditional transfer matrix calculation for beam transport is not convenient because the complications of considering the effect of the earth's field make the transfer matrix much harder to derive analytically. The new idea is based on single particle transportation equations and the code is developed in Matlab. Before the algorithm is introduced, the definition of coordinates used in the simulation model is necessary. Two 2-D 
coordinates $s-x$ and $z-y$, with $10^{\circ}$ difference are involved. The $z-y$ corresponds to the injection region, from IC2 to PD. The positive $z$ is beam transport direction on the injection line; $y$ is the direction perpendicular to $z$ in the horizontal plane and the positive $y$ means beam centroid offsetting outwards the ring. The $s-x$ corresponds to the ring line section, i.e. from PD to RC2. The positive $s$ is the beam transport direction along the pipe and the positive $x$ implies that beam centroid deviates from the ring axis outwards the ring center in the horizontal plane. The two coordinates meet at the same origin located at the center of the pulsed dipole. In both coordinates, the definition of the beam centroid angle in the horizontal plane is the angle between the beam motive direction and the beam transport direction along the pipe; the positive sign of an angle means the beam angle deflects outwards the ring center.

To explain the numerical algorithm in the new simulation model, the beam transport in the $s-x$ coordinate is for an example. We have the following equations based on the traditional Newton laws equations of motions and the beam transport equations.

$$
\begin{gathered}
x "=\ddot{x} / v^{2} \\
\gamma m \ddot{x}=-q v B
\end{gathered}
$$

Then we get Equation (3.7) from (3.5) and (3.6). In equation (3.7), $v$ is the beam velocity and $B$ is the magnetic field; $\gamma$ is decided by $10 \mathrm{keV}$ electron beam, $m$ is electron mass and $q$ is electron charge.

$$
x^{\prime \prime}=\frac{-q B}{\gamma m v}
$$


In order to develop a code to track the beam trajectory based on leap-frog iteration method, Equation (3.7) is used to derive the relation of ith-step beam divergence and (i-1)th-step divergence. For the tiny step, we have the followings.

$$
\begin{aligned}
& x^{\prime \prime}=\frac{d x^{\prime}}{d s}=\frac{x_{i}^{\prime}-x_{i-1}^{\prime}}{d s} \\
& x_{i}^{\prime}-x_{i-1}^{\prime}=x^{\prime \prime} \cdot d s
\end{aligned}
$$

Then we replace the $x "$ with $\frac{-q B}{m v}$ according to Equation (3.7) and get:

$$
x_{i}^{\prime}=x_{i-1}^{\prime}+\left(\frac{-q B}{\gamma m v}\right) \cdot d s
$$

The (i+0.5)th-step beam X offset in the horizontal plane can be derived from (i-0.5)th-step X offset because of the tiny step, shown in Equation (3.13) and (3.14).

$$
\begin{gathered}
x{ }_{i}^{\prime}=\frac{d x}{d s} \\
d x=x_{i}^{\prime} \cdot d s \\
x_{i+0.5}=x_{i-0.5}+d x \\
x_{i+0.5}=x_{i-0.5}+x_{i}^{\prime} \cdot d s
\end{gathered}
$$

The (i+0.5)th-step beam centroid position can be calculated from (i-0.5)thstep according to the tiny step $d s$, shown by Equation (3.15).

$$
s_{i+0.5}=s_{i-0.5}+d s
$$

For the beam centroid angle in the $s-x \operatorname{called} \theta$, we have the following equations.

$$
\operatorname{tg} \theta_{i}=\frac{v_{x}}{v_{z}}=\frac{d x / d t}{d z / d t}=\frac{d x}{d z}=x_{i}^{\prime}
$$




$$
\theta_{i}=\operatorname{arctg}\left(x_{i}^{\prime}\right)
$$

Until now enough equations has been derived to calculate the beam centroid positions and angles in the horizontal plane for the new simulation model we

discussed in section 3.1. The beam centroid position $\left(s_{i+0.5}, x_{i+0.5}\right)$ can be calculated by iteration from the previous step $\left(s_{i-0.5}, x_{i-0.5}\right)$ which is known at the initial beam position and angle.

\subsubsection{Beam centroid calculations based on the more accurate and extended}

model

In the extended simulation model we described in the previous sections, shown in Figure 3.1, the beam centroid is set with an initial offset at IC2 and transports through the injection region, Y-section and ring sections until RC2 under the earth's field effect and the effects of magnets in the model. The beam centroid positions and angles along beam trajectory are captured, which will be analyzed and guide design of magnets for UMER multi-turn commissioning.

The beam centroid is set $\mathrm{Y}=-0.4 \mathrm{~mm}$, for example, the quadrupoles including Q5, Q6 and ring quads are set as their normal operation currents, same as we described in section 3.1.2. Please refer for details. The big quads in the Y-section, i.e. YQ and QR1 are modeled as $5.21 \mathrm{~cm}$ long hard-edge quads and specified with peak gradient of $-5.49 \mathrm{G} / \mathrm{cm}$ (horizontally defocusing quad) , $5.49 \mathrm{G} / \mathrm{m}$ (horizontally focusing quad), respectively. All the dipoles including the pulsed dipole, steering dipoles and ring dipoles are set with specific bending angles $\theta_{d}$. When the simulation 
runs, the beam centroid starts from IC2 and is tracked through the center of Q4 (so that Q4 has no effect on the beam centroid), then beam centroid is captured at several points in the model.

When we track the beam trajectory in the injection line, the difficult part is to calculate the trajectory from SD6, passing through the YQ to the pulsed dipole. Since the calculation idea is based on the transport equations, we must use the distance on transport axis $\mathrm{z}$ between magnets for the leap-frog calculation. Although we know the YQ location, the exact points on which the beam hits the left and right edge of YQ and the point at which the beam reaches the PD are unknown. Also the YQ is modeled as a thick lens with effective length of $5.21 \mathrm{~cm}$ and its central axis makes an angle of $10^{\circ}$ with injection line. So we do not know the important $\mathrm{z}$ distances for SD6 to YQ left edge, with YQ, and YQ right edge to the PD. A special iteration method is used in the calculation to find these three $\mathrm{z}$ distance necessary. Our basic idea is to set a reasonable range of $\mathrm{z}$ coordinate of the end point, then assume every $\mathrm{z}$ coordinate in the range as the end point $\mathrm{z}$ coordinate, and get the beam trajectory with this $\mathrm{z}$ coordinate, finally see if the end point of y coordinate has a matched geometric relation with the end point of $\mathrm{z}$ coordinate.

We use the beam transporting from SD6 to the left edge of YQ to describe the iteration approach. Figure 3.5 shows the central part of $\mathrm{Y}$ section and the beam trajectory in this section (the blue curve). 


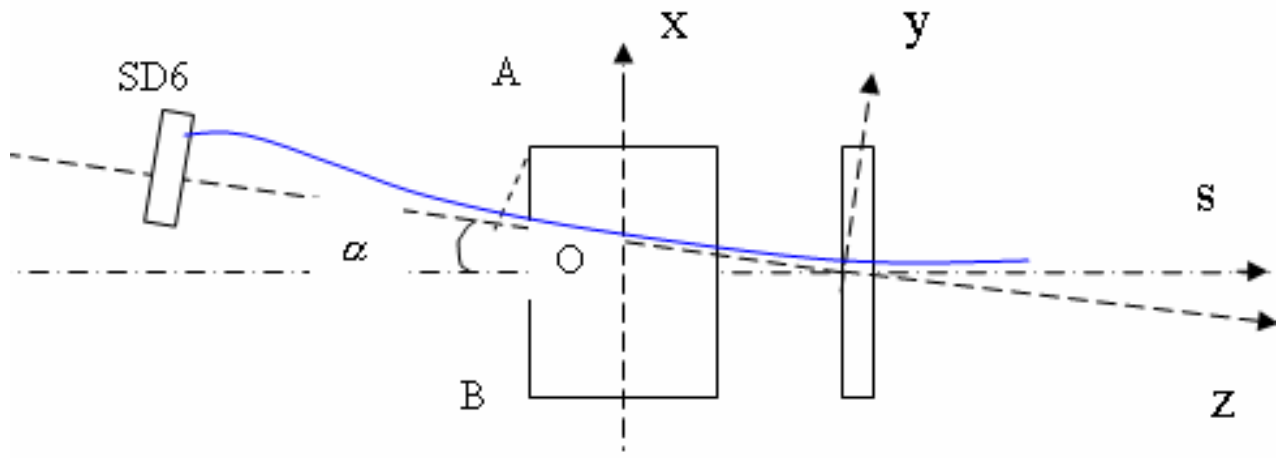

Figure 3.5 Schematic diagram of beam positions calculation in the more accurate model

Two kinds of beam trajectory are discussed here. Firstly, the beam enters the YQ through the top half, shown with the blue curve in Figure 3.5. We assume that the left edge of YQ crosses with the axis of injection line at point $\mathrm{O}$, and the $\mathrm{z}$ coordinate of point $\mathrm{O}$ can be obtained from the layout of $\mathrm{YQ}$ and called $z_{0}$. The trajectory enters the $\mathrm{YQ}$ at point $\mathrm{A}$ and the $z$ coordinate of point $\mathrm{A}$ is $z_{\mathrm{A}}$, which is unknown. We set a range of $z$ coordinate from $\operatorname{SD6}\left(z_{D}\right)$ to the midpoint of the $\mathrm{YQ}\left(z_{Q}\right)$, in which $z_{A}$ must be in the range. The $z$ coordinate between $z_{D}$ and $z_{Q}$ is iterated step by step (one step is $0.1 \mathrm{~mm}$ in the code). Each $z$ coordinate is set as the end point of the trajectory, and the $z$ distance from SD6 to the end point can be obtained and the beam trajectory can be tracked. The y coordinate of the end point can be obtained from the trajectory. The code checks the y coordinate, $\tan (\alpha)$ and $\Delta z$ (the distance between $z_{0}$ and the $z$ coordinate of the end point). From Figure 3.5, $\alpha$ is equal to $10^{\circ}$. The equation relation can be obtained. When $\Delta z=y \cdot \tan \alpha$, the iteration stops. The value of $z$ now is $z_{A}$, which is what we desire, the $z$ coordinate of the point at which the beam hits the left edge of YQ. 
On the other hand, when the beam centroid enters the YQ through the bottom half, the point the beam hits the left edge called point B. From the figure, the $z$ coordinate of point $\mathrm{B} z_{B}$ should be larger than $z_{O}$. After analysis, the approach we used above is applied correctly in this case. Thus the iteration method can be used no matter whether the beam hits the left or right half of the YQ. Moreover, we also can use the same iteration approach to find the exact point where the beam centroid exits the YQ from the right edge and where the beam centroid hits the pulsed dipole.

\subsection{Study of beam centroid motion at $\mathrm{RC2}$ due to the steering dipole}

\section{strength changing}

\subsubsection{Sensitivity study with Matlab simulation model}

The beam centroid sensitivity is defined as how much beam centroid offsets at a specific location corresponding to unit current increment of a steering dipole. The sensitivity of beam centroid positions downstream the Y-section, specified at RC2, corresponding to singly changing the strength of the steering dipole upstream the $\mathrm{Y}$, SD5h or SD6h is an interesting issue. The calculation for the sensitivity is introduced by the example of scanning SD5h strength. The calculation is based on the model shown in Figure 3.1, and settings of all the magnets are same with above settings in Section 3.2.2. The initial beam centroid position at IC2 is also $\mathrm{Y}=-0.4 \mathrm{~mm}$.

First, the bending angle of SD5h is scanned by a step of $-0.3323^{\circ}$ in the range of $4.9733^{\circ}$ to $1.9882^{\circ}$. The positive angle means the beam centroid deflects outwards the ring center horizontally. The scan range corresponds to $-1.5 \mathrm{~A}$ to $-0.6 \mathrm{~A}$, and the 
step is $0.1 \mathrm{~A}$. The negative current means that the dipole bends the beam outwards the ring in the horizontal plane. The beam centroid positions at $\mathrm{RC} 2$ corresponding to each strength of SD5h are captured and plotted in Figure 3.6. SD6h bending angle is fixed at $-1.2261^{\circ}$, corresponding to $2 \mathrm{~A}$, when SD5h is scanned.

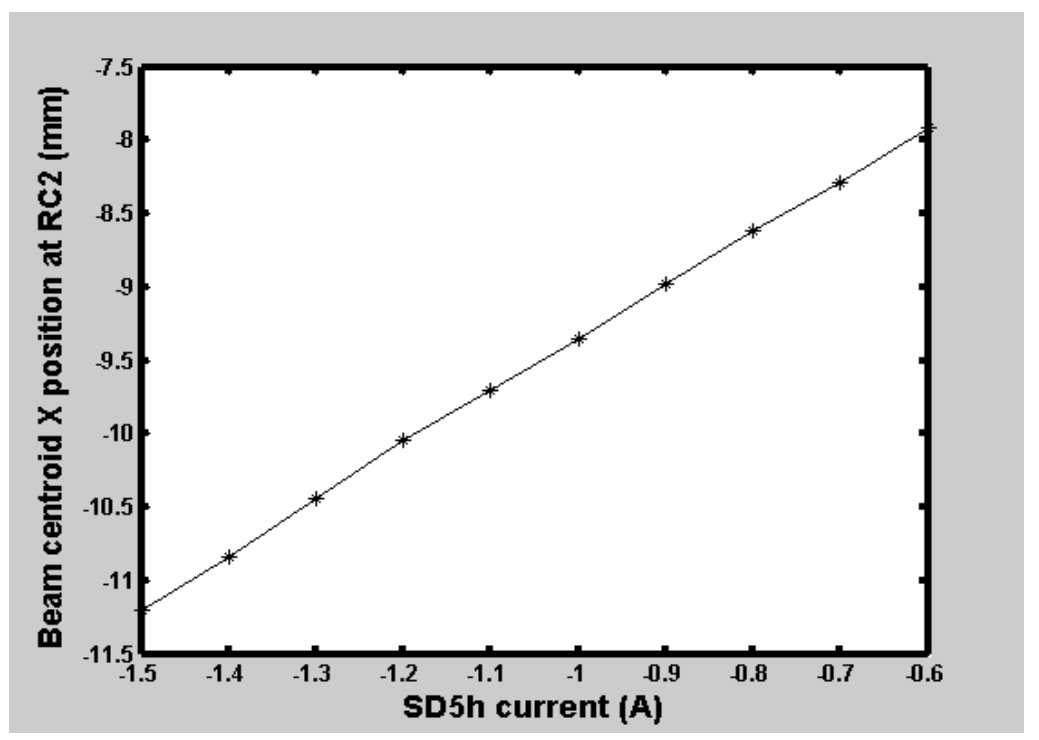

Figure 3.6 Sensitivity of the beam centroid at RC2 due to the SD5h scan in the Matlab calculation

Second, SD6h strength is scanned from $-0.6131^{\circ}$ to $-1.2261^{\circ}$ with stable setting of SD5h at $3.4114^{\circ}$. The corresponding scan range in strength is $1 \mathrm{~A}$ to $2 \mathrm{~A}$. The captured beam centroid positions at RC2 are plotted in Figure 3.7. The results of both SD5h and SD6h scans will be analyzed and compared with WARP simulation results later in this section. 


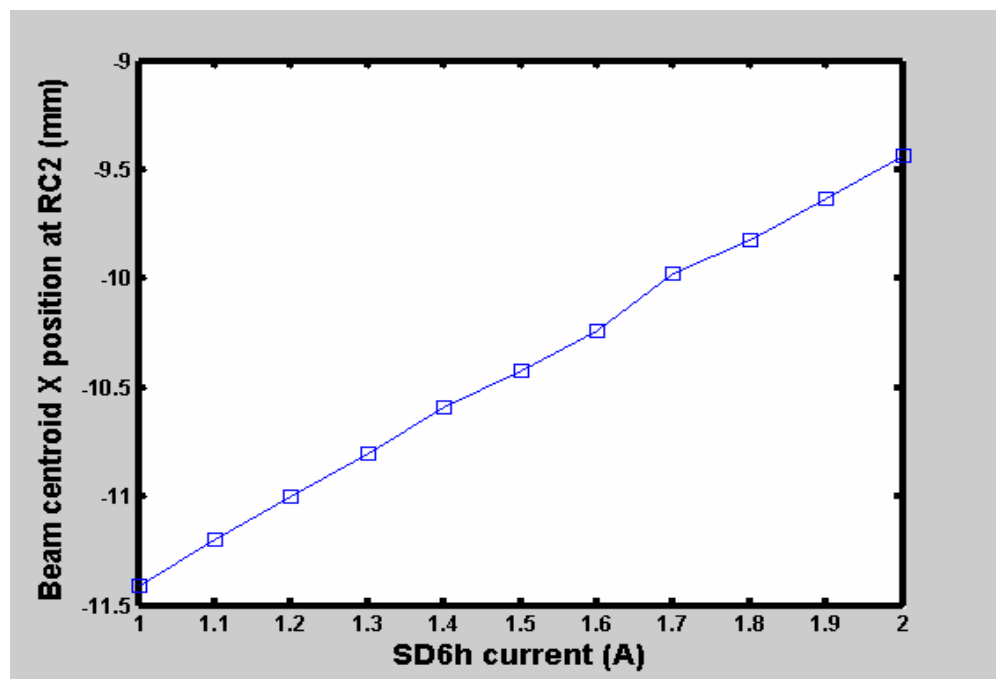

Figure 3.7 Sensitivity of the beam centroid at RC2 due to the SD6h scan in the Matlab calculation

\subsubsection{Sensitivity study in WARP simulation containing space charge}

The Matlab simulation is based on single particle theory so that the space charge is ignored in the Matlab simulation. The space charge, however, always exists and may arouse the image charge which affects beam centroid motion and the sensitivity to the steering dipole. So the WARP PIC simulation is run [11, 12, 24], which can conveniently include the space charge and image charge, to display the effects from them. The WARP simulation code was developed to study space charge beams, whose principle models are the PIC models with different dimensionalities. In this section, a two-dimensional axial PIC model, i.e. Warp XY is implemented to simulate the beam centroid evolution.

To express the space charge effect clearly, two beams with different currents are used in WARP simulations, one is $0.6 \mathrm{~mA}$ electron beam considering no space charge and the other is $24 \mathrm{~mA}$ beam with space charge. Key numerical settings for these two cases are different and shown in the following table. 
Table 3.4 WARP simulation parameters for beams with/without space charge

\begin{tabular}{ccccc}
\hline Beam current $/ \mathrm{mA}$ & Perveance & Emittance $/ \mathrm{mm} \cdot \mathrm{mrad}$ & Cells number & Particles number \\
0.6 & $9.03 \mathrm{e}-6$ & 5.5 & 32 & 4000 \\
24 & $3.61 \mathrm{e}-4$ & 30 & 256 & 40000 \\
\hline
\end{tabular}

For both two cases, the initial beam centroid position at IC2 location is set Y $=-0.4 \mathrm{~mm}$ and also the centroid is led to the center of Q4, that is has be done in Matlab simulation. The definitions of coordinates in the WARP simulation model is as same as coordinate definition in previous Matlab model. Then the beam centroid is evolved and captured at RC2 location.

In order to scan the SD5h strength or SD6h strength, another WARP code is developed to implement automatically setting the strength for the chosen steering dipole step by step and call the code tracking the beam centroid. The increment of both steering dipole scan is set as $0.1 \mathrm{~A}$.

The SD5h and SD6h are scanned in a specific range separately and the beam centroid is tracked and $\mathrm{X}$ position is captured at $\mathrm{RC} 2$. The simulation procedures are same with the procedure of scanning in the Matlab simulation. Please refer the previous section 3.2.2 for details. The differences are: the strength scan range for SD5h in the WARP simulation is $-1.5 \mathrm{~A}$ to $-0.6 \mathrm{~A}$, corresponding to the range of $4.9733^{\circ}$ to $1.9882^{\circ}$ in the Matlab simulation; for SD6h, the strength scan range is $1 \mathrm{~A}$ to $2 \mathrm{~A}$ instead of the range of $-0.6131^{\circ}$ to $-1.2261^{\circ}$ in the Matlab simulation.

In the WARP simulations, both $0.6 \mathrm{~mA}$ beam (no space charge) and $24 \mathrm{~mA}$ beam (space charge) are run for SD5h scan and SD6h scan, respectively. The beam centroid positions at RC2 location for SD5h scanning are plotted in Figure 3.8: the 
$0.6 \mathrm{~mA}$ simulation results are represented by the red stars; the $24 \mathrm{~mA}$ results are represented by the blue squares.

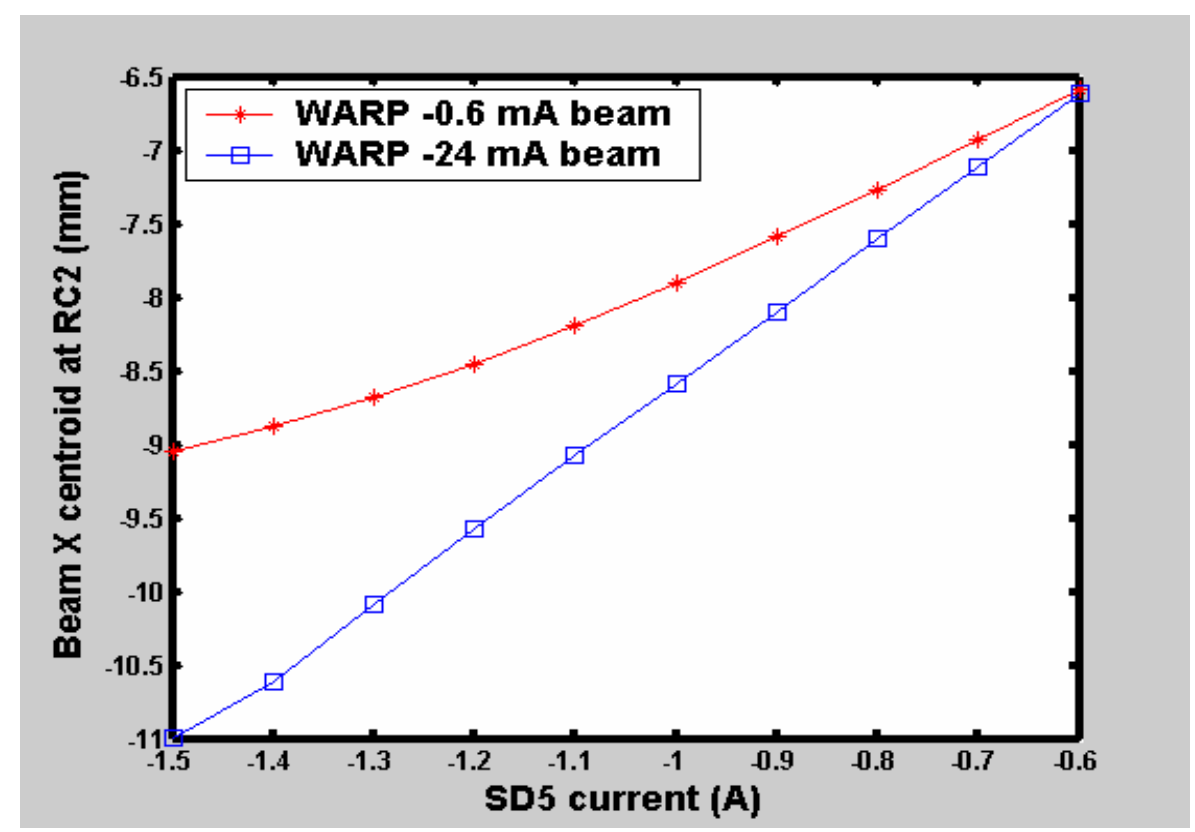

Figure 3.8 Sensitivity of the beam centroid at RC2 due to the SD5h scan in the WARP simulations

The simulation results for SD6h scan are in Figure 3.9: the red circles represents $0.6 \mathrm{~mA}$ beam and the blue triangles represents $24 \mathrm{~mA}$ beam. Analysis of the WARP simulation results and comparison with the Matlab results are followed. 


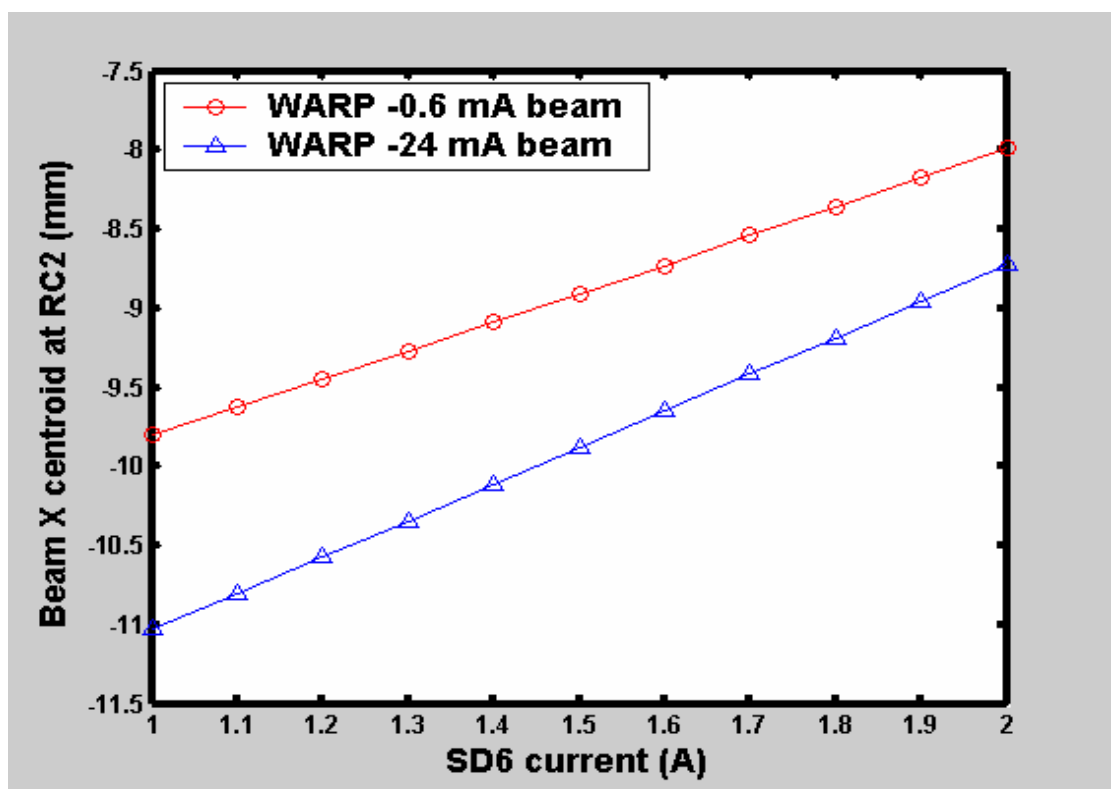

Figure 3.9 Sensitivity of the beam centroid at RC2 due to the SD6h scan in the WARP simulations

\subsubsection{Comparison and analysis of simulation results between Matlab and}

\section{WARP}

The simulation results from the Matlab model and both two WARP models are analyzed and compared in this section. For both SD5h and SD6h scans, the results from Matlab and WARP simulations are done by linear fit, respectively. We define the best fit slope as sensitivity. The best fit lines and the simulation results for SD5h and SD6h are shown in Figure 3.10 and Figure 3.11, respectively: the Matlab results and best fit line are represented by the blue stars and the blue line; the $0.6 \mathrm{~mA}$ WARP results and best fit line are represented by the red circles and the red line; the $24 \mathrm{~mA}$ WARP results and best fit line are represented by the green angles and the green line. From the best fit lines, good linearity of the results from all the simulations can be observed no matter for SD5h scan or SD6 scan. 


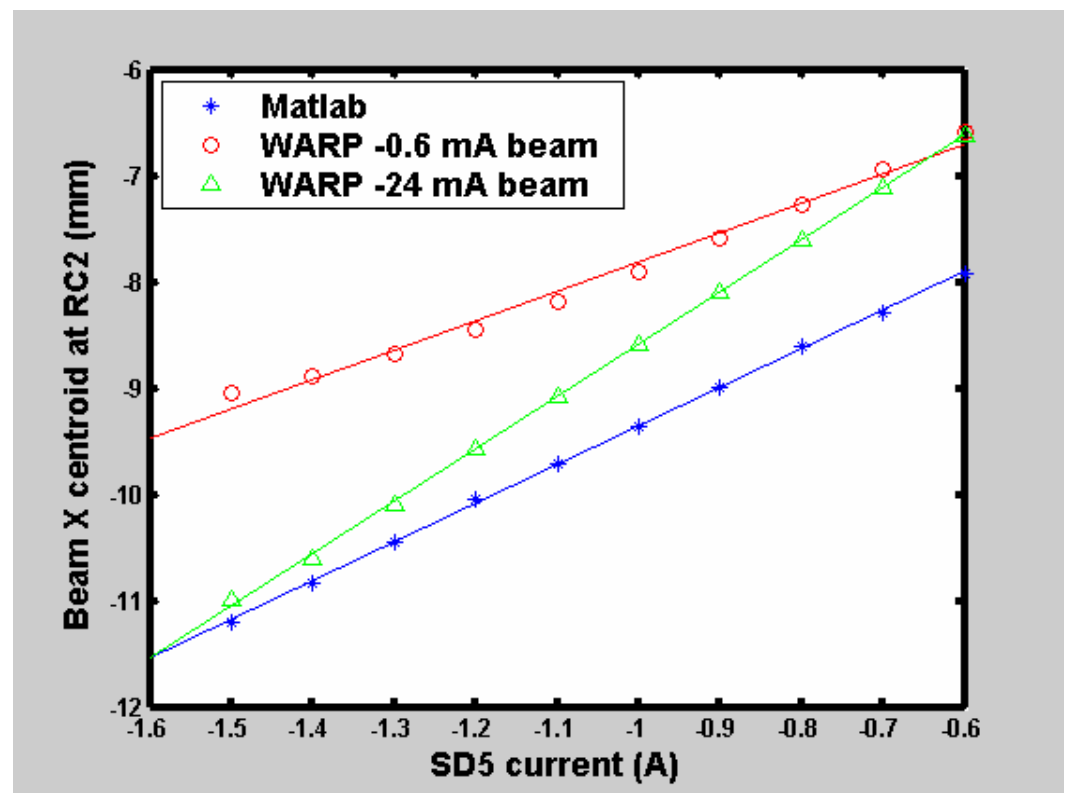

Figure 3.10 Best fit analysis and comparison of the beam centroid at RC2 due to SD5h scan between Matlab and WARP simulations

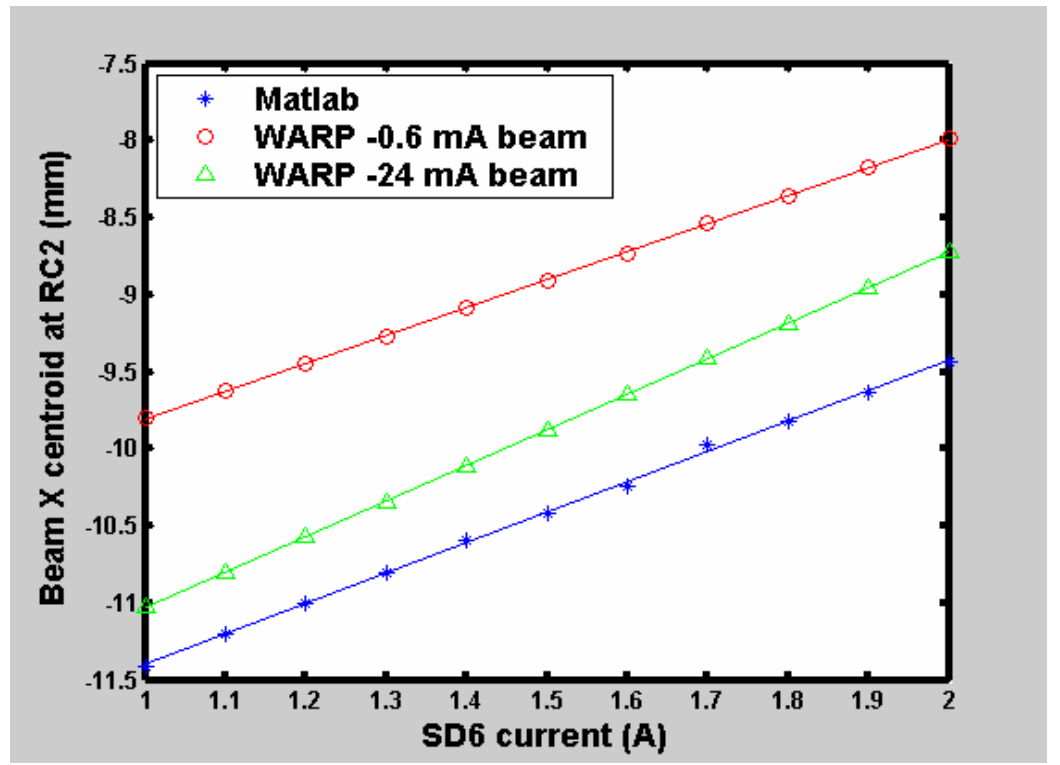

Figure 3.11 Best fit analysis and comparison of the beam centroid at RC2 due to SD6h scan between Matlab and WARP simulations

What we are mainly concerned with are the slopes of the best lines, which reflect the sensitivities for SD5h and SD6h scans. As shown in Figure 3.10 and 3.11, 
the results show good linearity of the sensitivity for both SD5h and SD6h. All the slopes of best fit lines of simulation results for SD5h and SD6h are shown in Table 3.5 .

Table 3.5 Sensitivities of beam centroid for SD5h and SD6h scans in Matlab and WAPR simulations

\begin{tabular}{|l|lcc|}
\hline & Matlab & $0.6 \mathrm{~mA}-\mathrm{WARP}$ & 24mA-WARP \\
\hline SD5h sensitivity $(\mathrm{mm} / \mathrm{A})$ & 3.64 & 2.76 & 4.92 \\
\hline SD6h sensitivity $(\mathrm{mm} / \mathrm{A})$ & 1.97 & 1.81 & 2.31 \\
\hline
\end{tabular}

For both SD5h and SD6h scans, the sensitivities from Matlab results show very good agreements with $0.6 \mathrm{~mA}$ WARP results, respectively. This is predicted by the simulation setups of the two simulations: the $0.6 \mathrm{~mA}$ WARP simulation hardly contains the space charge; the Matlab simulation is based on single particle transportation without space charge consideration also.

Next, for SD5h and SD6 respectively, we compare the sensitivity of $24 \mathrm{~mA}$ WARP simulation, containing the space charge effect, to the other two simulations without space charge, to determine if the space charge consideration impacts the beam centroid motion. As shown in Table 3.5, the sensitivity of $24 \mathrm{~mA}$ WARP simulation is larger than that of the other two simulations for both SD5h and SD6, meaning the space charge containing can affect the centroid sensitivity and more space charge results in larger sensitivity. 


\subsection{Calculation of the pulsed dipole settings based on the more accurate}

\section{simulation model}

The challenge for UMER multi-turn commissioning is to correctly design the pulsed dipole and set correct strengths for the pulsed dipole when the beam injecting and recirculating into the ring. In this section, a simulation study based on the more accurate model and Matlab algorithm we discussed earlier in this chapter is implemented. The estimated settings of the pulsed dipole to make the beam inject and return to the ring successfully will be shown.

\subsubsection{Beam centroid trajectory for one turn in the ring with WARP simulation}

In the improved model simulation discussed in Chapter 2, we led the beam to locate at the right edge of the pulsed dipole with zero $\mathrm{X}$ offset and zero angle because we think that the beam in the ring passes through PD perfectly, that means the beam is on the ring axis and perpendicular to transverse cross-section at the location of the pulsed dipole right edge. In reality, however, it is difficult to track the electron beam exactly the same with the trajectory in the simulation because of many reasons such as mechanical errors, the errors on magnet settings or unstable earth field.

In order to see how the beam transports in a more realistic magnetic model, a WARP simulation is used to track the beam centroid trajectory in the whole ring. The WARP simulation can calculate the magnetic field at a specific point due to involved magnets so that the beam transport in this simulation is described more accurately. The entire ring is composed of 36 lattices (a ring dipole, a focusing quadrupole and a defocusing quadrupole separated by the ring dipole), set up along the ring 
periodically. The simulation idea is: running the beam from the beginning point just before YQ with initial position and divergence $\left(x_{0}, x_{0}^{\prime}\right)$; after one turn, we make the beam hit the beginning point with same position and divergence, which means $\left(x, x^{\prime}\right)$ $=\left(x_{0}, x_{0}^{\prime}\right)$. The beam centroid trajectory is shown in Figure 3.12; the lower figure indicates the beam centroid trajectory expanded in order to see it clearly. The $\mathrm{S}$ is the direction that beam centroid transports in the ring; $\mathrm{X}$ is the beam centroid offset in the horizontal plane.
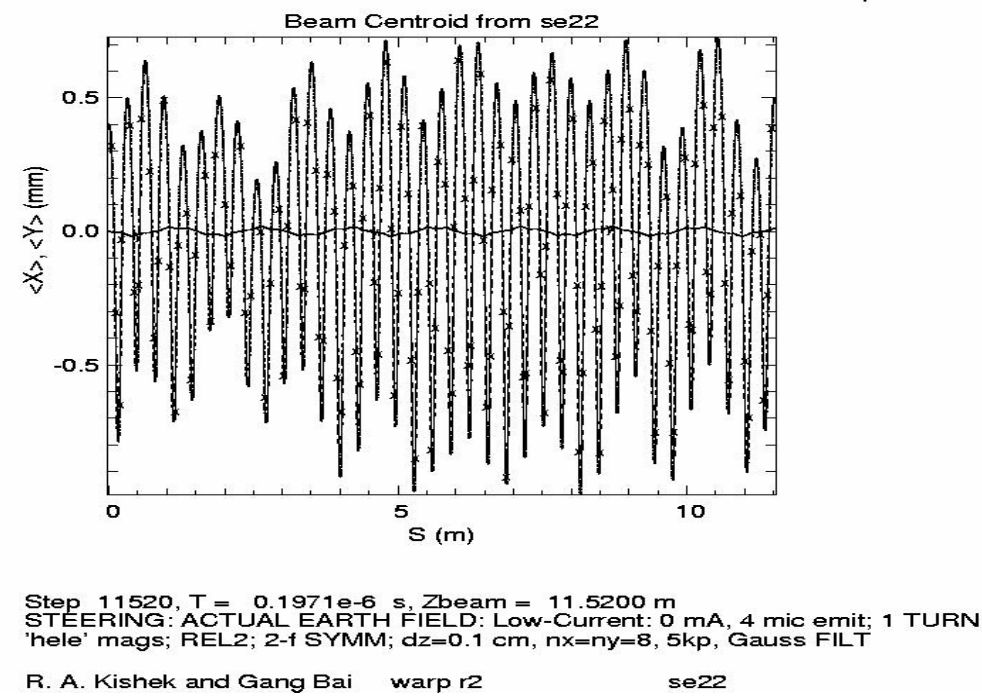

Figure 3.12 Beam centroid for one turn of the ring with WARP simulation

From the above figure, we can see that the beam centroid transports with periodic fluctuations in the horizontal plane because of the unstable effect from the earth's fields, which varies at different locations along the ring. The horizontal $x$ offsets fluctuate at dipoles about $0.5 \mathrm{~mm}$. This beam centoird trajectory gives us estimated beam centroid positions at dipoles under the effect of periodically set magnets and the earth's field. In other words, according to the beam centroid 
trajectory from the above WARP simulation, we can estimate that the beam X offsets in the horizontal plane at dipoles are $0.5 \mathrm{~mm}$.

\subsubsection{Study on the pulsed dipole setting when the beam injects into the ring}

The setting of the pulsed dipole for the beam injection into the ring is studied at this section. The more accurate model discussed in section 3.1.1, including the overlapped Y-section modeling, is used here but several magnets' modeling are modified. These include: Q5 and Q6 are modeled as thin quads, the beam transfer matrix of a thin quad is shown as Equation (3.3); the settings of $\mathrm{YQ}$ and $\mathrm{QR} 1$ are 5.50 $\mathrm{G} / \mathrm{cm}$ as effective gradient, respectively; the negative sign means horizontally defocusing the beam. The beam centroid is calculated in this model with a Matlab algorithm based on single particle transportation equations. The definitions of coordinates used and beam angle are same as the model in section 3.1.1: the coordinate $z-y$ corresponds to the injection line; $s-x$ is for the ring circle. The positive beam angle implies the beam is deflected outwards the ring center.

In the UMER experiment, when the beam centroid passes through both centers of the QR1 and QR2, the beam is considered to stream the ring correctly, which is also our goal in the simulation study, shown in Figure 3.13. The blue curve and red curve represent the injecting and returning beam, respectively. 


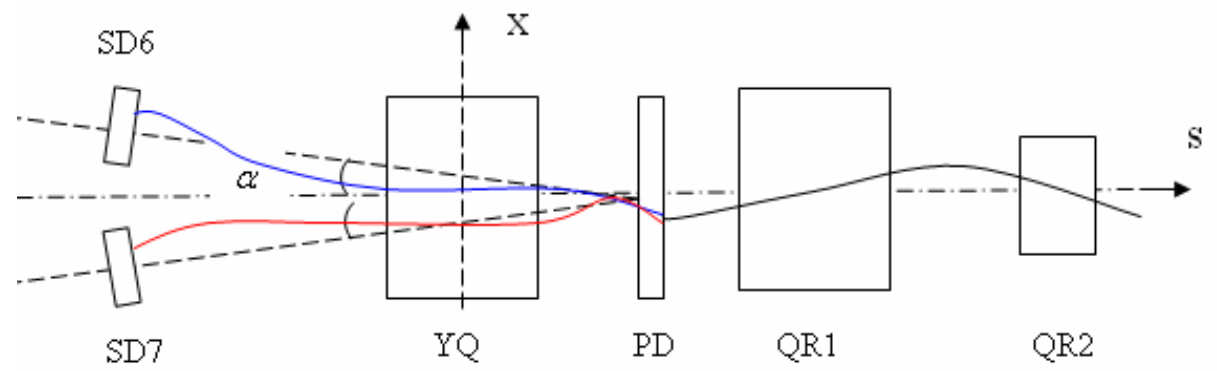

Figure 3.13 Schematic beam centroid trajectories for injection and recirculation cases in Y-section

Therefore, first, a Matlab code is developed to iterate the beam centroid position and exit angle at the pulsed dipole, and make the beam pass through both the centers of $\mathrm{QR} 1$ and $\mathrm{QR} 2$. We find that the beam should exit from PD at $-1 \mathrm{~mm} \mathrm{X}$ offset with the angle of $1.05^{\circ}$ to implement the expected centroid trajectory, shown in Figure 3.14. The beam centroid passes $\mathrm{X}=0$ at both $\mathrm{S}=8 \mathrm{~cm}$ where the $\mathrm{QR} 1$ locates and $\mathrm{S}=24$ where the $\mathrm{QR} 2$ locates. The exit angle $\theta_{\text {out }}=1.05$ is in the $s-x$ coordinate.

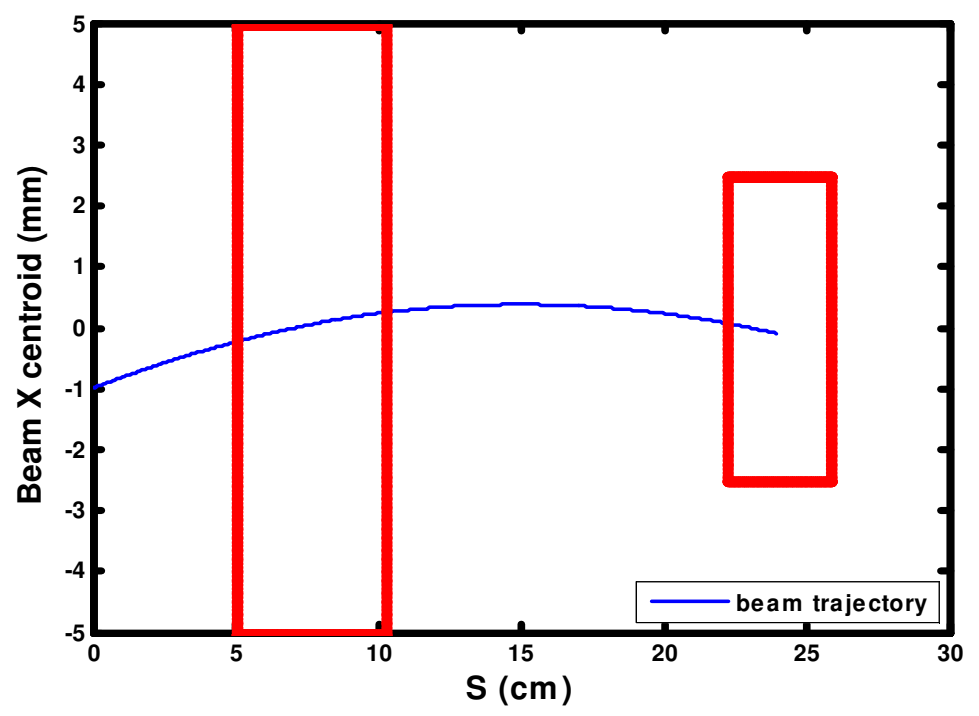

Figure 3.14 Beam centroid in the ring part passing through centers of QR1 and QR2 
Second, beginning at SD4h, we try to lead the beam centroid to hit the point of $\mathrm{X}=-1 \mathrm{~mm}$ at the $\mathrm{PD}$ after passing through the injection line, and capture the beam incidence angle of the PD. The initial beam centroid position is set at $y_{0}=0.5 \mathrm{~mm}$ at $\mathrm{SD} 4 \mathrm{~h}$, which is acquired from the estimated beam centroid in the WARP simulation of section 3.4.1.

The procedure details are: the initial beam centroid angle $x^{\prime}$ is scanned to make the beam pass through the center of Q5; then we scan the bending angle of SD5h to make the beam pass through the center of Q6; similarly, the bending angle of SD6h is scanned to make the beam hit the point of $\mathrm{X}=-1 \mathrm{~mm}$ offset at the pulsed dipole. As a result, the beam centroid incidence angle at the PD, $\theta_{i n}=2.02 \mathrm{deg}$ in the $z-y$ coordinate.

Based on the beam centroid exit angle $\theta_{\text {out }}$ and incidence angle $\theta_{\text {in }}$, the bending angle by the pulsed dipole is calculated and set, which can make the beam centroid successfully inject into the ring. Please note that $\theta_{\text {out }}$ is in the $s-x$ coordinate; $\theta_{\text {in }}$ is in the $z-y$ coordinate, which rotates $10^{\circ}$ clockwise to $s-x$ coordinate. So the bending angle of the pulsed dipole is calculated by $\theta_{p d}=\theta_{\text {out }}-\left(\theta_{i n}+\alpha\right)=9.03^{\circ}$. $\alpha=-10^{\circ}$, and the negative sign means clockwise rotation.

With the following Equation (3.18), the pulsed dipole strengths can be calculated.

$$
I=\frac{\theta_{p d} \cdot \gamma \cdot m \cdot \beta \cdot c}{q \cdot \text { Int.Field }}
$$


$\gamma, \beta$ are 1.0195 and 0.1948 respectively for a $10 \mathrm{keV}$ beam; $\mathrm{m}$ is electron mass, $c$ is speed of light, $\mathrm{q}$ is unit charge; Integral field of the pulsed dipole is 2 $\mathrm{Gcm} / \mathrm{A}$.

The result is that when the pulsed dipole is set as $-26.71 \mathrm{~A}$, the beam can inject into the ring successfully. The negative sign implies the pulsed dipole bends the beam outwards the ring center in the horizontal plane.

\subsubsection{Returning leg study based on the more accurate model}

The injection/recirculating section (Y-section) is designed for beam multi-turn operation. This section not only steers the beam from the injection line to the ring, but also makes the beam correctly return to the ring again after one turn and repeat under the effect of the designed magnetic fields. According to the design of the pulsed dipole, the beam centroid is bent outwards the ring when beam injecting while oppositely towards the ring when returning. The pulsed dipole changes its polarity timely according to the pulsed supplies.

One point worthy to mention is that the asymmetric effect of the earth field's on the low-energy beam when the beam injects and returns back through the $\mathrm{Y}$ section. Since the earth's field at the UMER location is almost vertically down, the electron beam is bent only towards to the ring center in the horizontal plane. This means that the earth's field helps bend the beam when the beam returns to the ring while having the opposite effect when the beam is injected into the ring. So it is expected that the strength of the pulsed dipole designed for returning beam should be less than that for injecting the beam into the ring. The calculation of the pulsed dipole strengths for beam injecting and returning is followed in this section. 
Figure 3.15 shows the model we use, which is a part of the more accurate and extended model in Section 3.1.1, including the returning steering dipole SD7h. The red curve is returning beam trajectory. The SD7h is perpendicular to the returning line and $19.99 \mathrm{~cm}$ apart from the center of pulsed dipole. The returning line is also $10^{\circ}$ different with the ring line but located symmetrically with the injection line; a new coordinate $z^{\prime}-y^{\prime}$ is used for the returning line. The layouts of other magnets are same as the model in section 3.1.1, containing the overlapped field consideration. The YQ is modeled as a hard-edge big quadrupole: the effective length of which is $5.21 \mathrm{~cm}$ and the integral gradient is $5.50 \mathrm{Gauss} / \mathrm{cm}$; the pulsed dipole is considered as a thin dipole; the earth's field is still modeled as 0.4 Gauss, vertically down magnetic field in the model. The definitions of coordinates used and the beam centroid angle in the horizontal plane are same as the model in section 3.1.1.

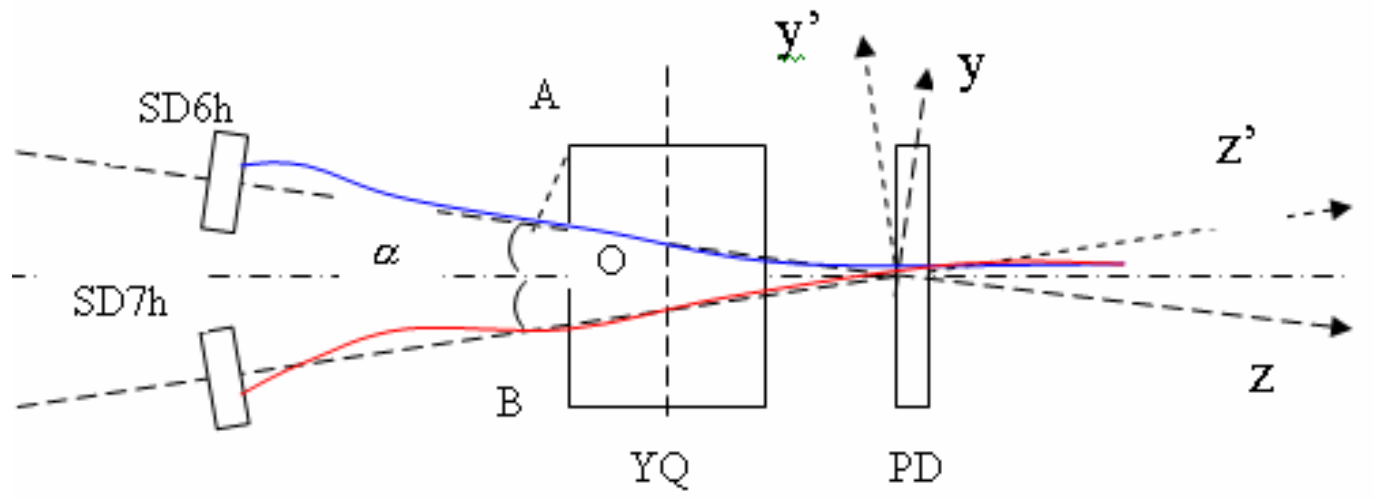

Figure 3.15 Schematic layout of returning leg for the calculation pf PD setting for returning case

From the simulation results from section 3.4.2, in order to make the beam centroid pass through both centers of $\mathrm{QR} 1$ and $\mathrm{QR} 2$, the beam should exit from the pulsed dipole at $-1 \mathrm{~mm}$ with an angle of $\theta_{\text {out }}=1.05^{\circ}$. At this time, a Matlab procedure 
is developed to track the beam starting from SD7h, pass the YQ and hit the pulsed dipole at $-1 \mathrm{~mm} \mathrm{X}$ offset. The incidence angle of the beam centroid into the PD is captured. Based on the ideal exit angle $\theta_{\text {out }}$, the bending angle of the pulsed dipole for correctly returning the beam is acquired.

We now set initial beam centroid $Y^{\prime}$ positions $0.5 \mathrm{~mm}$ at $\mathrm{SD} 7 \mathrm{~h}$. The initial beam centroid $Y^{\prime}$ position is from the WARP estimation in section 3.4.1. When the beam centroid enters the pulsed dipole at $-1 \mathrm{~mm}$ point, the incidence angle is $\theta_{i n}=-4.16^{\circ}$ in $z^{\prime}-y^{\prime}$ coordinate. The negative sign of angle means the beam deflects from the axis towards to the ring center.

Because the coordinate $z^{\prime}-y^{\prime}$ rotates $10^{\circ}$ counterclockwise with the ring line coordinate $s-x$, the bending angle of the pulsed dipole can be calculated by $\theta_{p d}=\theta_{\text {out }}-\left(\theta_{\text {in }}+\alpha\right), \alpha=10^{\circ}$ for counterclockwise rotation; $\theta_{\text {out }}=1.05 \mathrm{deg}$. The result is that $\theta_{p d}=-4.79^{\circ}$ for $y^{\prime}=0.5 \mathrm{~mm}$ initial beam position from returning leg.

With Equation (3.18), the corresponding current setting of the pulsed dipole to return the beam is $+14.17 \mathrm{~A}$. The positive sign means that the pulsed dipole deflects the beam towards the ring center horizontally.

\subsubsection{Comparison between Matlab simulation results on the pulsed dipole settings for injecting/returning beam with the experiment}

In UMER experiment, the injection quads and ring quads are powered with their normal strengths; the YQ and QR1 are set as -5.45 A, 5.45 A respectively, corresponding to the $\mp 5.50 \mathrm{G} / \mathrm{cm}$ peak gradient. The experimental process implements the multi-turn control algorithm to make beam pass through both centers 
of QR1 and QR2 for both injecting and recirculating beams. The experimental result of the pulsed dipole setting is $-27.5 \mathrm{~A}$ for injection and 14.0 A for recirculation.

The experiment results are compared with the PD settings calculated from the Matlab simulation, shown in Table 3.6. The simulation results show good agreement with the experiments for both injecting and returning beam.

Table 3.6 Settings of the pulsed dipole for injecting and returning beam in experiment and Matlab simulations

\begin{tabular}{cccc}
\hline & $\theta_{\mathrm{pd}}($ simulation $)$ & Simulation & Experiment \\
\hline Injection & $9.03^{\circ}$ & $-26.71 \mathrm{~A}$ & $-27.5 \mathrm{~A}$ \\
\hline Returning & $-4.79^{\circ}$ & $14.17 \mathrm{~A}$ & $14.0 \mathrm{~A}$ \\
\hline
\end{tabular}

The positive bending angle and negative current imply that the pulsed dipole deflects the beam outwards the ring center in the horizontal plane; the opposite sign means that the pulsed dipole bends the beam towards the ring.

From the above table, we can conclude that (1) the polarity of pulsed dipole is reversed between injected and returning beam. When the beam is injected to the ring, the PD bends the beam outwards the ring center in the horizontal plane; it bends the beam towards the ring center when the beam returns to the ring. This is agreed with the UMER design. (2) The PD strength for beam returning is smaller than that for injecting the beam. The reason is that the earth's field pointing vertically down resists the bending of the pulsed dipole when the beam is injected to the ring while assisting the PD bending when the beam returns. These results are good guides for the design of the pulsed dipole. 


\section{Chapter 4 Experiment Study on Y-section}

\section{$\underline{4.1 \text { Experiment setup for study of injection/recirculating section }}$}

\subsubsection{Introduction}

The experiment is implemented on the facility of University of Maryland Electron Ring (UMER) [7]. The schematic of the whole experimental system is shown in the Figure 4.1.

The experiment is designed to study the Y-section focusing on the beam centroid motion. The Y-section, however, is a black box without any diagnostics, thus we choose the injection line, the $\mathrm{Y}$ section and the first two ring sections in this experiment. Therefore we now have three diagnostics chambers IC2 (Injection Chamber) and RC1 \& RC2 to obtain information of the beam.

An automatic control system is developed for the experiment, which is illustrated in Figure 4.1. It involves two computers, connected with Ethernet for communication. Computer I, operated by Linux, is the core of the control system, responsible for controlling quadrupole powers through serial communication, commanding the switch controller to choose the BPM signal via GPIB, acquiring the beam centroid information from the chosen BPM via Oscilloscope I; Computer II is controlling powers of all the dipoles. The automatic control system not only allows us to operate the machine from the computer screen, but also implement acquisition and analysis of the key beam parameter, i.e. centroid position. 


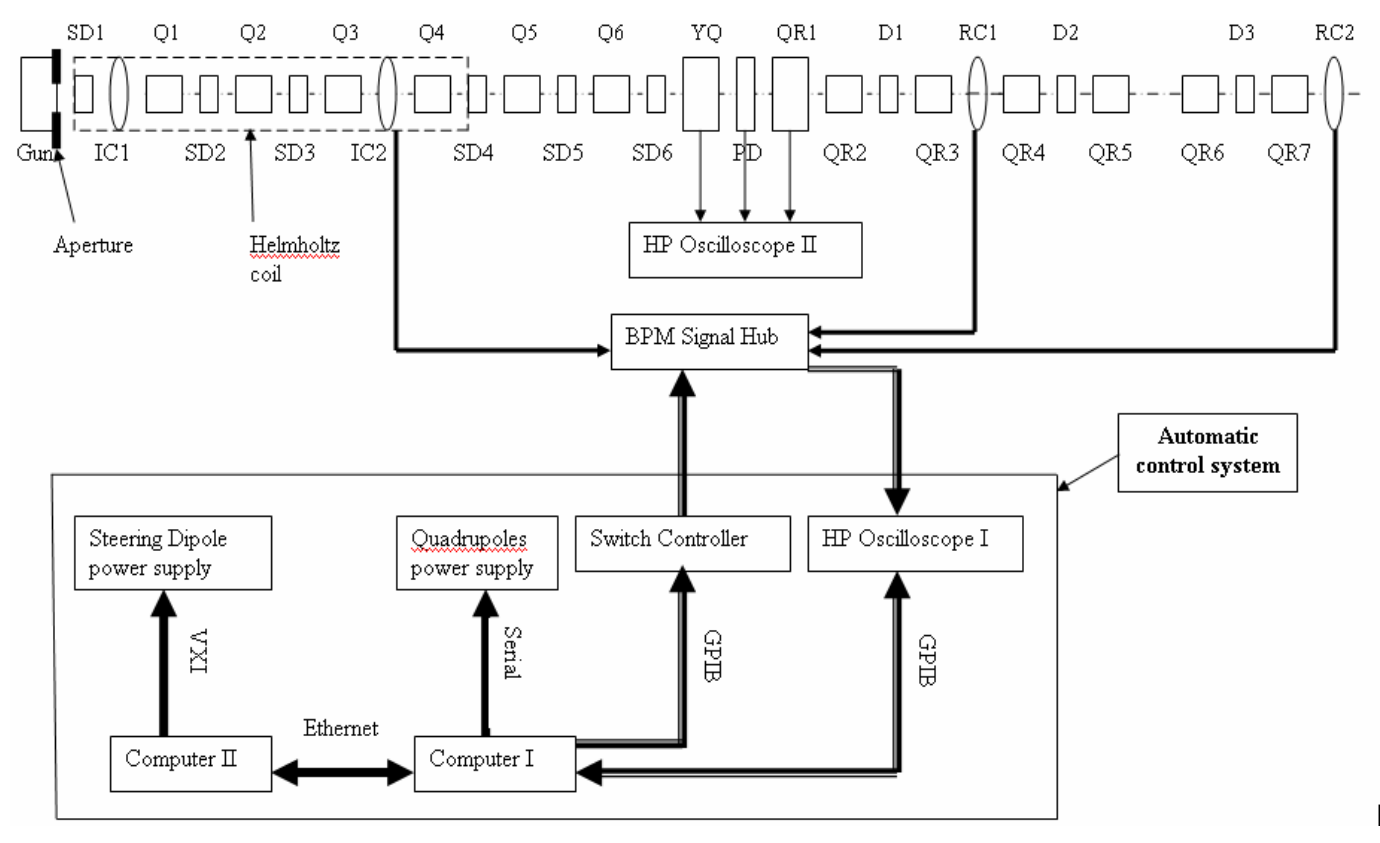

Figure 4.1 Schematic layout of the experimental system

The injection line in a distance of approximately $1.3 \mathrm{~m}$, is composed of the electron gun, six steering dipoles (SD1-SD6), six independent powered quadruples, two diagnostics chambers (IC1 and IC2) and a Bergoz fast current transformer, also Helmholtz coils.

We use a typical B-type thermionic dispenser cathode gun to provide electron beams on UMER. This gun can generate a low-energy of $10 \mathrm{keV}$, high-current of 100 $\mathrm{mA}$ and $100 \mathrm{~ns}$ pulse electron beam. The electron gun's power is provided by a high voltage power supply. The photoemission properties of this kind of cathode were studied by B. Leblond in the 1990's [25].

An adjustable aperture is located just downstream of the electron gun. By adjusting it, we can obtain an extensive range of beam current ( $0.6 \mathrm{~mA}$ to $100 \mathrm{~mA})$. A short solenoid is used after the aperture to focus the beam in the matching solution. Between the solenoid and SD1, approximately $62 \mathrm{~cm}$ from the electron gun, a Bergoz 
fast current transformer is set to measure the $24 \mathrm{~mA}$ rectangular beam current in realtime (the beam mainly used in this experiment). The measured rise time is $2.8 \mathrm{~ns}$. The Bergoz model number is FCT-082-20:1.

The steering dipoles are adjusted individually in both horizontal and vertical directions, to make the beam transport on the expected trajectory in the injection line and enter the Y section at the point we expect. The six quadrupoles are independently powered with Lambda Zup power supply, and set up focusing and defocusing in turn (Q1 is defocusing). The group of quadrupoles is used as individually quadrupole scanning in the steering program, also used to match the beam into the ring. The Helmholtz coil is set up on the injection line (from the aperture to SD4), powered by HP E3610A DC supply. This coil is tilted to cancel the earth field both in horizontal and vertical directions. The current of the Helmholtz coil is $0.64 \mathrm{~A}$ to cancel the earth's field at the location of the UMER injection line in Maryland.

The $\mathrm{Y}$ section we use in the experiment is the part of the injection section, which includes SD6, YQ (Y-section big quadrupole) and PD (the pulsed dipole), also QR1 (the first ring quadrupole). As we mentioned in the previous chapter, YQ, PD and QR1 form the first lattice of the ring; YQ and QR1 are on two sides of PD with the same distance to PD (both are $8 \mathrm{~cm}$ ); YQ and QR1 are designed with the same effective length (both are $5.38 \mathrm{~cm}$ ) and the same peak on-axis gradient (both are 1.01 $\mathrm{G} / \mathrm{cm} / \mathrm{A}$ ). In the experiment, we set YQ with a positive current of 5.45 A while QR1 is set at a current of negative 5.45 A so that $\mathrm{YQ}$ works as a horizontal defocusing magnet and QR1 is a horizontal focusing magnet. For the pulsed dipole (PD), we set its current of -24 A in the experiment. As we discussed before, the PD is powered by 
two different pulses: one is a long pulse with a strength of -40 A for the returning beam; the other is a short pulse of $16 \mathrm{~A}$, which is added to the long pulse when the beam is injected so the strength of the pulsed dipole for injecting the beam is $-24 \mathrm{~A}$.

Also, we use the first three lattices of the ring and the second ring chamber (RC2). The BPM set in RC2 is the most important diagnostic in the experiment to monitor the beam positions.

Since the results of this experiment will be compared with the results from the calculation we discussed in the previous chapter, the current setting of several magnets such as YQ, QR1 and PD, must be very accurate and kept without any change during the whole experiment. We also need to keep the beam current the same during the experiment in order to maintain the beam condition unchanged. So a HP Infinium oscilloscope is used to monitor the signals of three high voltage power supplies for $\mathrm{YQ}, \mathrm{QR} 1$ and PD. All of these three signals are provided to the magnets, respectively, while input into the oscilloscope for detecting during the experiment. Moreover, the signal out of the Bergoz fast current transformer is also input into the oscilloscope to be checked.

The signals from BPMs are sent to a hub and chosen by a Tektronix 1360P programmable switch controller to be forwarded into HP Oscilloscope II. The switch controller, which is controlled by a computer through GPIB card, decides which BPM's signal to be sent to the Oscilloscope I. The signals in the Oscilloscope I can be acquired by the computer through another GPIB card and processed by the algorithm developed by Labview, output information of the beam centroid [26]. 


\subsubsection{Description of the BPM}

The beam position monitors (BPM) and phosphor screens are the main beam diagnostic in UMER. Eighteen diagnostics chambers are distributed along the ring, in which both BPMs and phosphor screens are housed. The Figure 4.2 shows the schematic layout of a diagnostics chamber (the phosphor screen is under the BPM).

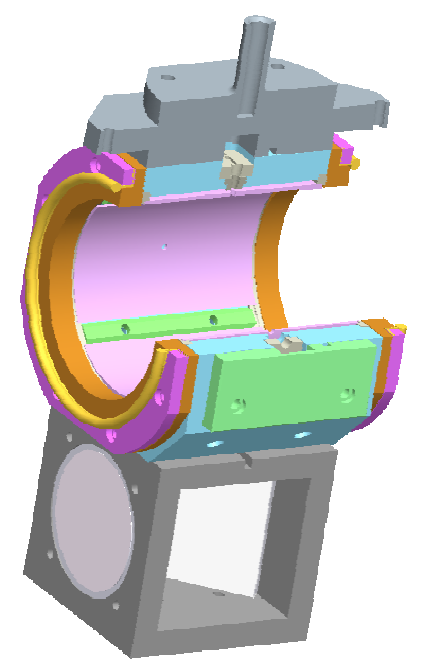

Figure 4.2 Layout of a ring chamber with BPM and P-screen housed

In the previous experiment, the phosphor screen was chosen for beam diagnostics because phosphor screen can provide beam images for beam matching, and accurate information for beam steering with relative beam positions [6]. In our experiments, the absolute horizontal beam position in IC2 must be obtained and used as the initial beam position in the calculation. All the BPMs used in UMER have been calibrated accurately and the relation equations transferring the signals from a BPM to absolute beam positions in both horizontal and vertical direction have been found. So we choose BPMs for our beam diagnostics in experiments. 
The BPM can provide four signals at the same time, which are used to evaluate the beam position. The four signals are $\mathrm{T}$ (top), B (bottom), L (left), R (right), in which $\mathrm{T}$ and $\mathrm{B}$ are the signals of the top and bottom plates in BPM, reflecting beam vertical position while $\mathrm{L}$ and $\mathrm{R}$ show the beam horizontal position. The equations to calculate beam centroid position from corresponding BPM signals are acquired from BPM calibrations [27]. The basic idea of calibrating one BPM is to measure a group of the BPM signals and corresponding beam positions, then do a best fit of the data. The equation describing the best fit line is used to calculate the real beam centroid position. The best fit line is shown as $Y=m \cdot X+B$. Y is normalized value for BPM signals: $Y=(R-L) /(R+L)$ for the horizontal plane; $Y=(B-T) /(B+T)$ for the vertical plane. The $X$ is real beam centroid position horizontally or vertically. The resolution $m$ is the change of the $\mathrm{Y}$ value per unit change of $X(1 \mathrm{~mm})$, which is actually the slope of the best fit line; B is the offset of the best fit line.

The calibrations of BPMs housed in IC2, RC1 and RC2 are done in both horizontal and vertical planes. The results are shown in Table 4.1.

Table 4.1 Several BPM calibration results

\begin{tabular}{|l|c|c|c|c|c|c|}
\hline & \multicolumn{2}{|c|}{ IC2 } & \multicolumn{2}{c|}{ RC1 } & \multicolumn{2}{c|}{ RC2 } \\
\hline & Offset & Resolution & Offset & Resolution & Offset & Resolution \\
\hline Horizontal & -0.0223 & 0.0804 & 0.0305 & 0.0838 & -0.002 & 0.0839 \\
\hline Vertical & -0.0503 & 0.0796 & 0.1314 & 0.0751 & 0.0305 & 0.787 \\
\hline
\end{tabular}


The calculation of the beam centroid X position horizontally at IC2 based on the horizontal calibration of IC2 BPM is introduced as an example. According to the offset $\mathrm{B}=-0.0223$ and the resolution $m=0.0804$, the best fit line equation is $Y=0.0804 \cdot X-0.0223$. So the beam centroid position in the horizontal plane is calculated by the equation of $X=(Y+0.0223) / 0.0804$; the unit of $\mathrm{X}$ is millimeter; the positive $\mathrm{X}$ means beam centroid deflects towards the ring according to the definition of BPM calibration. In the experiment, we can read the BPM signals of R and L at IC2 from the oscilloscope and input into the control computer. The input data is processed by the designed program and outputs the horizontal normalization of $\mathrm{R}-\mathrm{L} / \mathrm{R}+\mathrm{L}$. Then the beam centroid $\mathrm{X}$ position at IC2 can be obtained by the above equation. The beam centroid positions at other BPMs are calculated with same idea based on the normalized BPM signals.

\subsubsection{Pulsed Dipoles and Quadrupoles in Y-section}

The steering dipoles (SD1-SD6) and independent powered quadrupoles (Q1-Q6) in the injection line, and the ring dipoles and ring quadrupoles of the ring FODOs are general printed-circuit (PC) magnets powered by DC supplies. The pulsed dipole is specially powered by long and short pulses based on the requirement of injection/recirculation switch. Considering the compatibility and symmetry, the big YQ and QR1 located in Y section should also be powered by pulses. The general design characters of the pulsed dipole and quadrupoles are shown in Table 4.2 and Table 4.3, respectively. 
Table 4.2 Key design characters of the pulsed dipole

\begin{tabular}{cc}
\hline Peak field & $0.386 \mathrm{G} / \mathrm{A}$ \\
Operation Current & $27 \mathrm{~A}$ \\
Effective length & $5.18 \mathrm{~cm}$ \\
Integral field & $2.0 \mathrm{Gcm} / \mathrm{A}$ \\
Transverse alignment error & $<0.05 \mathrm{~mm}$ \\
\hline
\end{tabular}

Table 4.3 Key design characters of the big DC quadrupole

\begin{tabular}{cc}
\hline Peak gradient & $1.01 \mathrm{G} / \mathrm{cm} / \mathrm{A}$ \\
Operation Current & $5.45 \mathrm{~A}$ \\
Effective length & $5.38 \mathrm{~cm}$ \\
Integral gradient & $5.43 \mathrm{G} / \mathrm{A}$ \\
Transverse alignment error & $<0.05 \mathrm{~mm}$ \\
\hline
\end{tabular}

\subsection{Key experimental device settings}

UMER can provide a broad range of current beams from $0.6 \mathrm{~mA}$ to $100 \mathrm{~mA}$. In the experiment, a $24 \mathrm{~mA}$ beam is chosen (the aperture after the electron gun is $230^{\circ}$ correspondingly). The $24 \mathrm{~mA}$ beam is shown by the purple line in Figure 4.3.

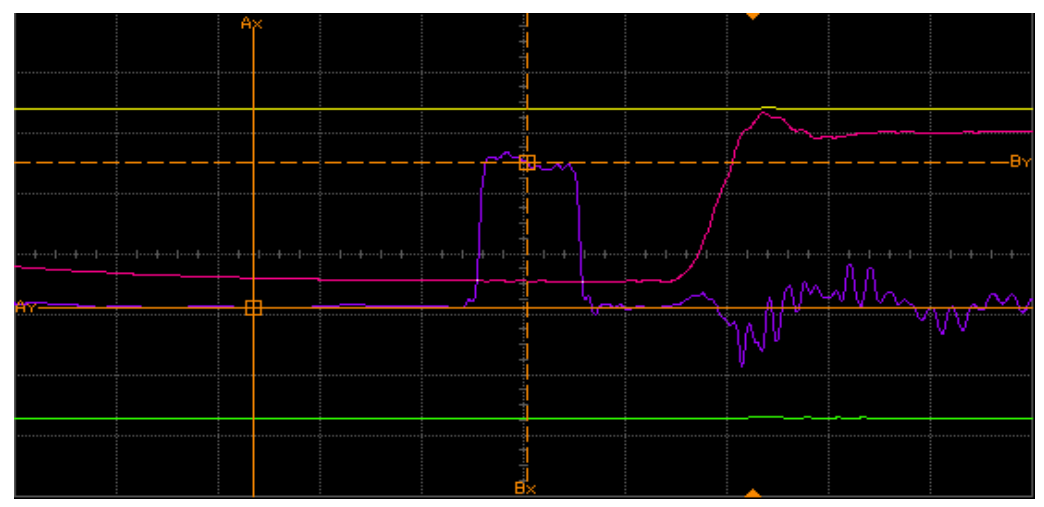

Figure 4.3 Experimental setting of $24 \mathrm{~mA}$ beam 
The Helmholtz coils are used in the injection line and the whole ring to cancel the earth's field. We have two DC powers: one is set at $0.64 \mathrm{~A}$, the other is set at 0.15 A. All components of the earth's field are canceled in the injection line (before SD4); while only the horizontal component of the earth's field is canceled in the ring.

The important issue is setting power supplies for the pulsed magnets in the $\mathrm{Y}$ section which is YQ, QR1 and pulsed dipole. The settings of these magnets are monitored by a HP oscilloscope, shown in Figure 4.4. The yellow line and the green line indicate the settings of YQ and QR1, respectively. The YQ and QR1 are powered with the same amplitude but opposite polarity. The red line represents the pulsed dipole current.

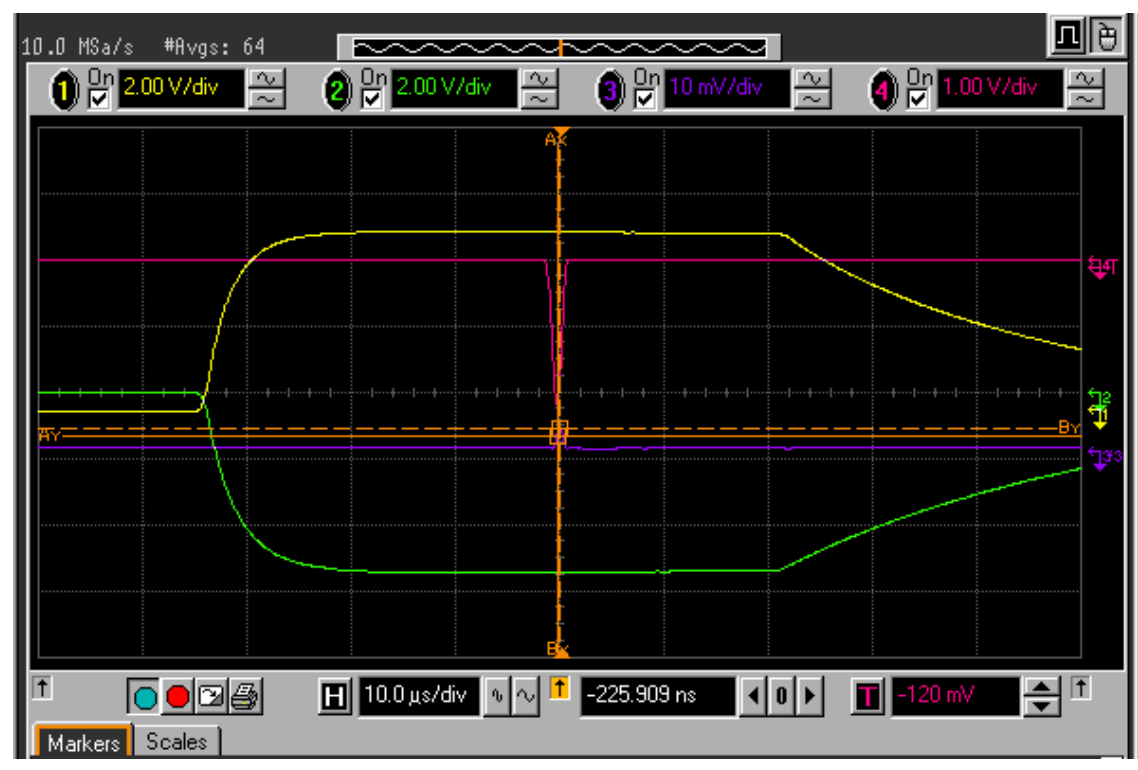

Figure 4.4 Experimental settings of YQ and QR1

The current settings for the pulsed magnets and other experimental settings are shown in Table 4.4. The negative sign of quad current means that the quadrupole defocuses the beam in the horizontal plane; the negative sign of the pulsed dipole current imply bending the beam outwards the ring center. 
Table 4.4 Several important experimental settings

\begin{tabular}{cc}
\hline YQ power supply & $-5.44 \mathrm{~A}$ \\
QR1 power supply & $5.45 \mathrm{~A}$ \\
Pulsed dipole injection & $-27 \mathrm{~A}$ \\
power supply & $24 \mathrm{~mA}$ \\
Injection line Bergoz coil & $0.76 \mathrm{~A}$ \\
\hline
\end{tabular}

\subsection{Experiment implementation}

The experiment is designed to study the Y-section and the experiment results will be compared with the Matlab and WARP simulation. The layout of all the magnets and diagnostics used in experiments is shown in Figure 4.5. The simulation model shown in Figure 3.1 in Chapter 3 originates from this layout. Please refer Chapter 3 for details of designed characters of involved magnets. As same as the definitions of both Matlab and WARP simulation models, the positive beam centroid $\mathrm{X}$ position in the experiment implies beam deflects outwards the ring center horizontally. 


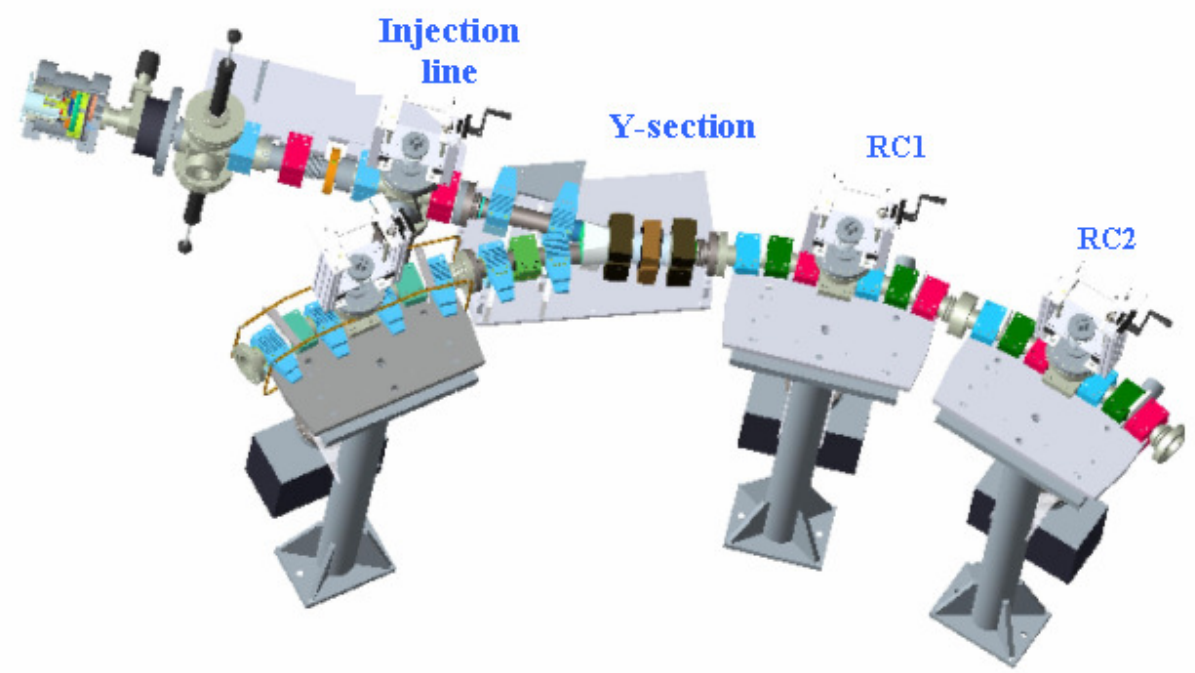

Figure 4.5 Layout of the experimental facility

\subsubsection{Obtain initial beam centroid $X$ position and angle}

In order to compare the beam status between the calculation and the experiment, the same initial beam position and momentum for both are necessary. Since in the simulation, the beam initial position, which is beam offset in horizontal at IC2, and the initial momentum can be set as what we expect while it is more difficult to do the same things in the experiment. Our idea is to find the initial beam position and momentum in the experiment firstly then input it to the simulation. The beam position at IC2 can be measured by the BPM based on the calibration of IC2 BPM while the difficulty is how to know the momentum of beam at IC2 because the BPM can only give us the beam position but no information on the momentum. That means we are not able to get the beam momentum directly from the experimental data. What we do is to make the beam pass through the center of the Q4, which is exactly downstream IC2, by scanning SD3. In the simulation, we set the beam initial position at IC2 as same as the centroid position we measured in the experiment, and then track 
beam passing the $\mathrm{Q} 4$ center. So we can get a rough same initial status on both beam position and momentum at IC2 in the experiment and simulation.

In the experiment, SD3 both horizontal and vertical, are separately scanned to find the strength settings that make beam centroid through the center of Q4 in the horizontal or vertical plane independently. An automatic program based on the idea of quadrupole scanning is developed in the Labview environment to find if the beam centroid passes through the quad center [26, 28]. The basic idea is that the strength of Q4 is scanned in a reasonable range (less than $0.5 \mathrm{~A}$ ), and the corresponding beam centroid positions of each set Q4 strength are measured at RC2. The beam position is represented by the normalized BPM signals in the experiment: $(\mathrm{R}-\mathrm{L}) /(\mathrm{R}+\mathrm{L})$ is for horizontal position; $(\mathrm{B}-\mathrm{T}) /(\mathrm{B}+\mathrm{T})$ is for vertical position as we discussed in section 4.1.2. A set of normalized signals from the horizontal or vertical scan are linearly fit, separately. The beam centroid is considered to pass through the Q4 center when the slope of the fit line is equal to zero.

A typical quad scan result in the Labview program is shown in Figure 4.6. Here both SD3h and SD3v are set with specific currents: SD3h $=-0.11 \mathrm{~A}$ and SD3v $=0.55 \mathrm{~A}$. The negative sign means bending the beam outwards the ring horizontally; Q4 is scanned and the scan range is $0.2 \mathrm{~A}$; the beam centroid positions at $\mathrm{RC} 2$ are shown as the graphs: the purple line represents the centroid horizontal positions; the green line represents the beam positions in the vertical plane. The linear fit slope of the horizontal scan is $-3.428 \mathrm{e}-3$ per A; for the vertical case, the fit line slope is $-3.69 \mathrm{e}-$ 3 per A. The scans of Q4 are done by several times to decrease the experimental errors. The averages of the slopes are $-0.75 \mathrm{e}-3$ per A, $2.38 \mathrm{e}-3$ per A for the horizontal 
scan and the vertical scan, respectively. Both the slopes for horizontal and vertical cases are small enough to say that the beam centroid passes through the center of Q4.

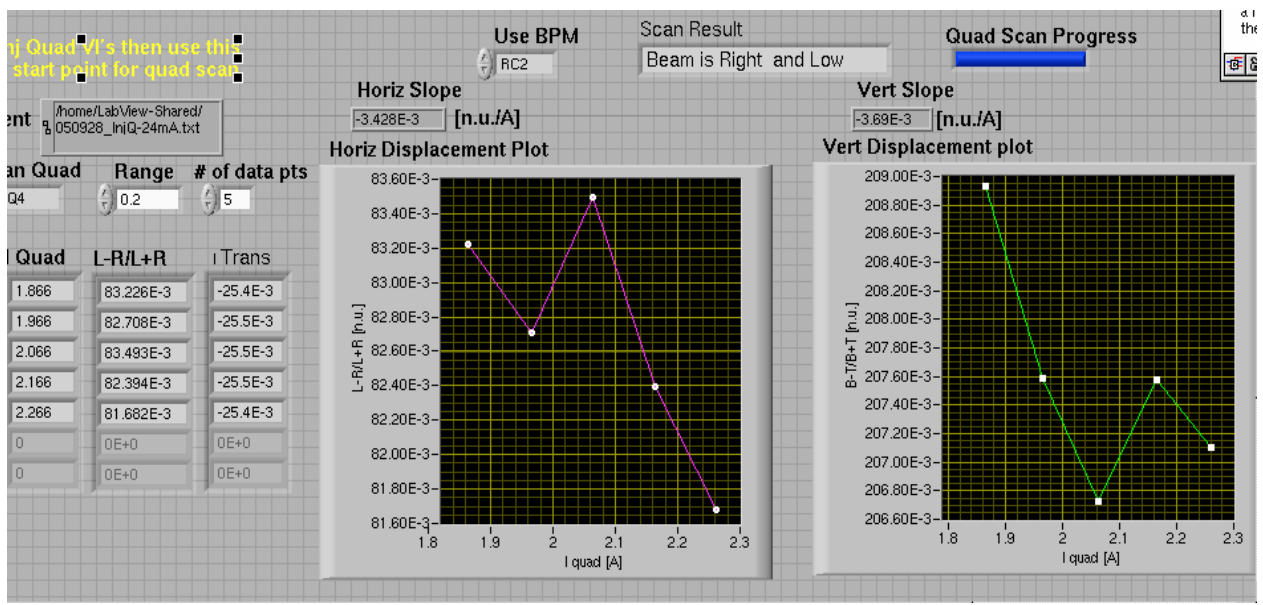

Figure 4.6 Typical quadrupole scan and results showing the beam centering the scanned quad

Next, the beam centroid horizontal position at IC2 is measured by the BPM housed in IC2. The normalized BPM signals $\mathrm{R}-\mathrm{L} / \mathrm{R}+\mathrm{L}$ can be measured directly from the experiment. In order to decrease the experimental errors, we take the measurement for more than 10 times and obtain the average value, which is $-8.3 e-3$ for $\mathrm{R}-\mathrm{L} / \mathrm{R}+\mathrm{L}$. The actual beam centroid $\mathrm{X}$ position at $\mathrm{IC} 2$ can be calculated by $X=(Y+0.0223) / 0.0804$. The calculated beam centroid $\mathrm{X}$ position at IC2 is $0.1741 \mathrm{~mm}$, which offsets the ring axis towards the ring center according to the definition of BPM calibration. So this $\mathrm{X}$ position in the experimental, Matlab and WARP definitions is $\mathrm{X}=-0.1741 \mathrm{~mm}$. The initial beam centroid position will be input into both simulations as initial status. 


\subsubsection{Experimental measurement of sensitivity of beam centroid at $R C 2$}

The basic idea of the experimental implementation is to independently scan the strength of one horizontal steering dipole while freeze other horizontal steering dipoles, and measure the beam $\mathrm{X}$ centroid position at $\mathrm{RC} 2$ for the each set strength; from the experimental data, we desire to compare the effect on beam centroid of the scanned steering dipoles. During the experiment, the involved magnets other than steering dipoles are frozen with their normal operating strengths, including the pulsed dipole PD, DC big quadrupoles $\mathrm{YQ}$ and $\mathrm{QR} 1$, the third generation quadrupoles Q5 and Q6, ring dipoles D1, D2, D3, the second generation quadrupole QR2-QR7 and vertical steering dipoles SD4v, SD5v, RSV1, RSV2. All the magnets' settings are shown Table 4.5. For dipoles, the negative current means beam is kicked outwards the ring center and positive current means towards the center; for quadrupoles, the negative current means the quadrupole defocusing beam in the horizontal plane while the positive current means focusing horizontally.

Table 4.5 Experimental settings of involved magnets

\begin{tabular}{cccccccccc}
\hline SD3h & SD3v & SD4h & SD4v & SD5v & RSV1 & RSV2 & D1 & D2 & D3 \\
$-0.11 \mathrm{~A}$ & $0.55 \mathrm{~A}$ & $0.13 \mathrm{~A}$ & $0.79 \mathrm{~A}$ & $0.03 \mathrm{~A}$ & $-0.5 \mathrm{~A}$ & $0.3 \mathrm{~A}$ & $2.1 \mathrm{~A}$ & $2.7 \mathrm{~A}$ & $2.8 \mathrm{~A}$ \\
\hline $\mathrm{Q} 3$ & $\mathrm{Q} 4$ & $\mathrm{Q} 5$ & $\mathrm{Q} 6$ & $\mathrm{QR} 2$ & $-\mathrm{QR} 7$ & $\mathrm{PD}$ \\
$2.13 \mathrm{~A}$ & $2.07 \mathrm{~A}$ & $2.11 \mathrm{~A}$ & $2.11 \mathrm{~A}$ & $1.88 \mathrm{~A}$ & $-27 \mathrm{~A}$ \\
\hline
\end{tabular}

Figure 4.7 (a) shows the RC2 beam centroid X positions when the SD5h (h is horizontal) is varied gradually by a step of $0.1 \mathrm{~A}$ while SD6h is frozen at $2 \mathrm{~A}$. The scan range is from -1.6 A to -0.5 A; Figure 4.7(b) is for SD6h scan while SD5h kept as $-1.029 \mathrm{~A}$; the scan range is from $1 \mathrm{~A}$ to $2 \mathrm{~A}$. The positive $\mathrm{X}$ centroid means that the 
beam centroid deflects from the ring axis outwards the ring center in the horizontal plane.

From the experimental data, we can see that the beam centroid $\mathrm{X}$ positions change linearly responding to the strength increment for SD5h or SD6h respectively; with the strengths of both steering dipoles increasing, the beam centroid at RC2 deflects outwards to the center of the ring.
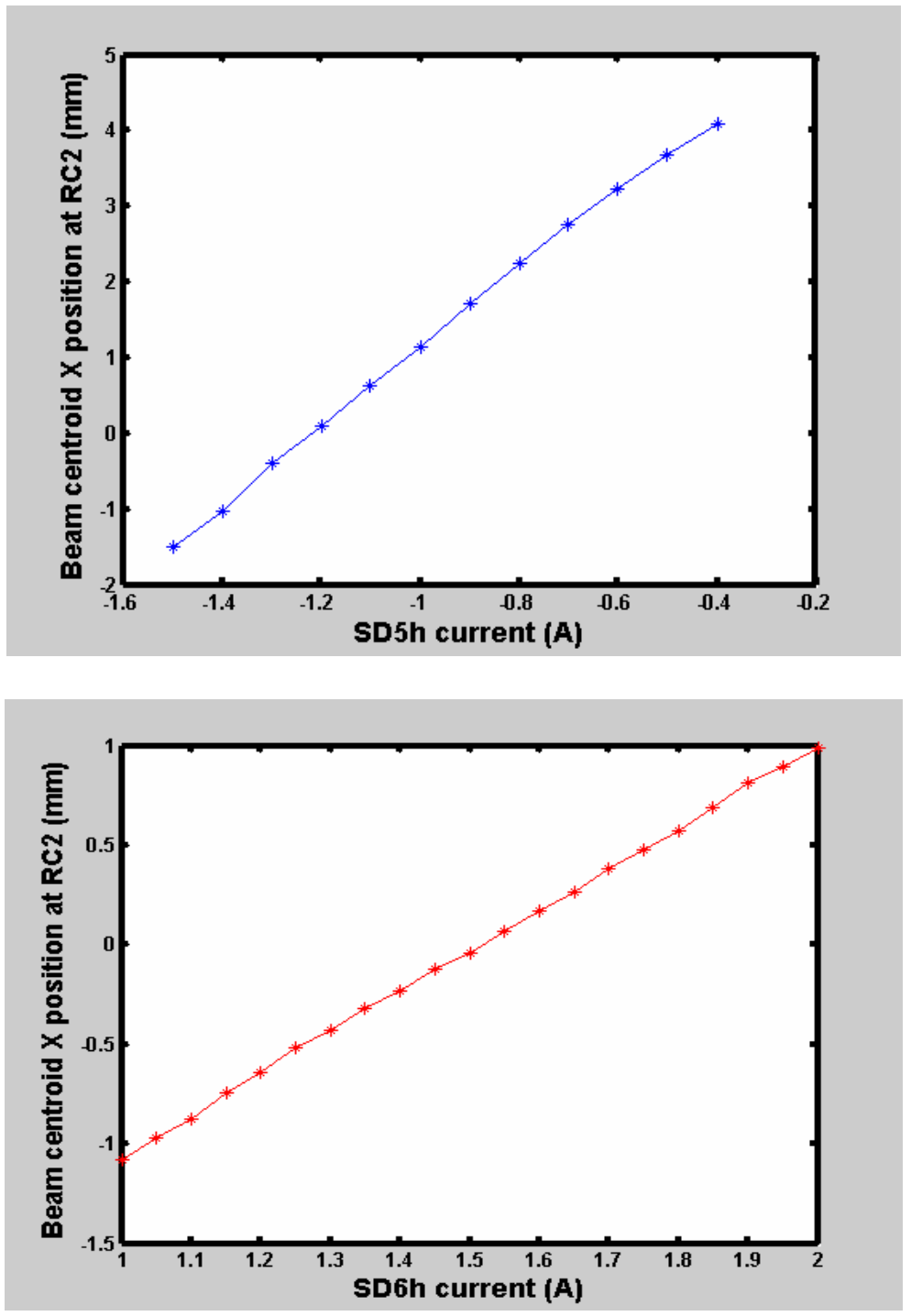

Figure 4.7 (a) Centroid sensitivity of $24 \mathrm{~mA}$ beam at RC2 due to the SD5h scan in the experiment (b) Centroid sensitivity of $24 \mathrm{~mA}$ beam at RC2 due to the SD6h scan in the experiment 


\section{$\underline{4.4 \text { Experiment result analysis and comparison with simulations }}$}

In this section, experiment results of scanning the SD5h and SD6h are analyzed in quantity. The initial beam centroid X position at IC2 obtained from the experiment is used as initial beam centroid position in both WARP and Matlab simulations and the results are analyzed as same as experiment results. The comparison among the results from the experiment, the WARP simulation and the Matlab simulation and analysis will be shown.

\subsubsection{Import the beam initial centroid into Matlab model}

In the experiment, the initial beam centroid $\mathrm{X}$ position at chamber IC2 has been acquired by the BPM, which is $-0.1741 \mathrm{~mm}$ and should be imported into the Matlab model we describe in the Chapter 3 (reference to Figure 3.1) to serve as the initial beam centroid position at IC2. The Matlab code can simulate beam centroid and indicates the beam centroid X position in the horizontal plane.

The Matlab code leads the beam centroid passing through the center of Q4 by scanning the exit angle from IC2. Then all the magnets in the simulation model are set as same strengths as the current settings in the experiment.

First, for YQ as an example, which is modeled as a $5.21 \mathrm{~cm}$ long magnet, the effective gradient defined in the simulation is $-5.4944 \mathrm{G} / \mathrm{cm}$ calculated by Gradient $_{\text {effective }}=$ Gradient $_{\text {peak }} \cdot I_{\text {operation }}$, I is equal to $-5.44 \mathrm{~A}$, the operation current of YQ in the experiment; the negative sign means the YQ defocusing beam horizontally; Gradient $_{\text {peak }}$ is peak gradient of YQ, $1.01 \mathrm{G} / \mathrm{cm} / \mathrm{A}$ from Table 4.3. The simulation setting of QR1 is acquired with similar method for $\mathrm{YQ}$, and is $5.5045 \mathrm{G} / \mathrm{cm}$ for 
amplitude but opposite polarity, which means focusing beam in the horizontal plane. The simulation method for Q5 and Q6 are different from YQ or QR1 because Q5 and Q6 are modeled as thick quadrupoles with $3.72 \mathrm{~cm}$ effective length, and the transfer matrix of this quadrupole is implemented to describe the beam centroid before/after the quadrupole (reference to Chapter 3 for details). For the operating current $-2.11 \mathrm{~A}$ for Q5 and 2.11 A Q6 from experiments, the force $\kappa_{Q 5}$ set in the simulation is $224.47 \mathrm{~m}^{-2}$ as defocusing beam in the horizontal plane; and $\kappa_{Q 6}$ is $224.47 \mathrm{~m}^{-2}$ for focusing beam horizontally. The calculation has been done in Chapter 3. Also, the ring quadrupoles QR2-QR7 are considered as thin quadrupoles in the simulation. Please refer Chapter 3 for details of ring quadrupole modeling and calculation. The focal length of horizontal defocusing ring quadrupoles (QR2, QR4, QR6) is $-11.97 \mathrm{~m}$ while the focal length of horizontal focusing ring quads (QR3, QR5, QR7) is $11.97 \mathrm{~m}$.

Second, since all the steering dipoles, the pulsed dipole, and ring dipoles are considered as thin dipoles in the Matlab simulation, the bending angles for every dipole powered by a specific current in the experiment (shown in Table 4.5) can be calculated by the following equation.

$$
\theta=\frac{I \cdot q \cdot \text { Int.Field }}{\gamma \cdot m \cdot \beta \cdot c}
$$

$\gamma, \beta$ are decided by $10 \mathrm{keV}$ electron beam, $m$ is electron mass, $c$ is speed of light, $q$ is electron charge. For example of the pulsed dipole, the experimental setting is $-27 \mathrm{~A}$ and the integral field is $2 \mathrm{Gcm} / \mathrm{A}$, so the bending angle of PD set in the simulation is $9.1307^{\circ}$. According to simulation model definition, the positive angle implies beam bent outwards the ring center, which matches the negative current 
setting for PD in the experiment. Similarly, bending angles set in the simulation for other steering dipoles and ring dipoles are obtained from Equation (4.1), which will be shown in Table 4.6. The integral field for SD4h, SD5h, SD6h are $3.886 \mathrm{Gcm} / \mathrm{A}$, 19.612 Gcm/A, and 3.627 Gcm/A; for ring dipoles D1, D2, D3, the integral field is $19.612 \mathrm{Gcm} / \mathrm{A}$.

Table 4.6 Bending angles of involved dipoles set in the Matlab simulation corresponding to experimental settings

\begin{tabular}{lcccc}
\hline SD4h & PD & D1 & D2 & D3 \\
$-0.086^{\circ}$ & $9.13^{\circ}$ & $-6.83^{\circ}$ & $-8.85^{\circ}$ & $-9.12^{\circ}$ \\
\hline
\end{tabular}

Along the simulation model described above, the beam centroid is tracked in the horizontal plane from the initial $\mathrm{X}$ position of $-0.1741 \mathrm{~mm}$. As same as the experiment, the beam centroid $\mathrm{X}$ positions at RC2 are captured while the bending angle of SD5h or SD6h is scanned separately.

When SD6h bending angle is fixed at $-1.226^{\circ}$ matching with $2 \mathrm{~A}$, the bending angle of SD5h is scanned by a step of $0.332^{\circ}$, which is -0.1 A step for SD5h scanning in the experiment. The scan range corresponds to $-0.6 \mathrm{~A}$ to $-1.5 \mathrm{~A}$ in the experiment. The beam centroid X positions for each SD5h bending angle are catch at RC2 and plotted in Figure 4.8 (a); Then SD5h bending angle is frozen at $3.411^{\circ}$ matching with -1.029 A, and scans SD6h bending angle by a step of $-0.063^{\circ}$, which is matched with 0.1 A step for SD6h. The scan range is for $1 \mathrm{~A}-2 \mathrm{~A}$ done in the experiment. The beam centroid X positions at RC2 for every setting of SD6h bending angle are plotted in Figure 4.8 (b). The Matlab simulation results will be analyzed and compared with experiment results, WARP simulation results later in this section. 

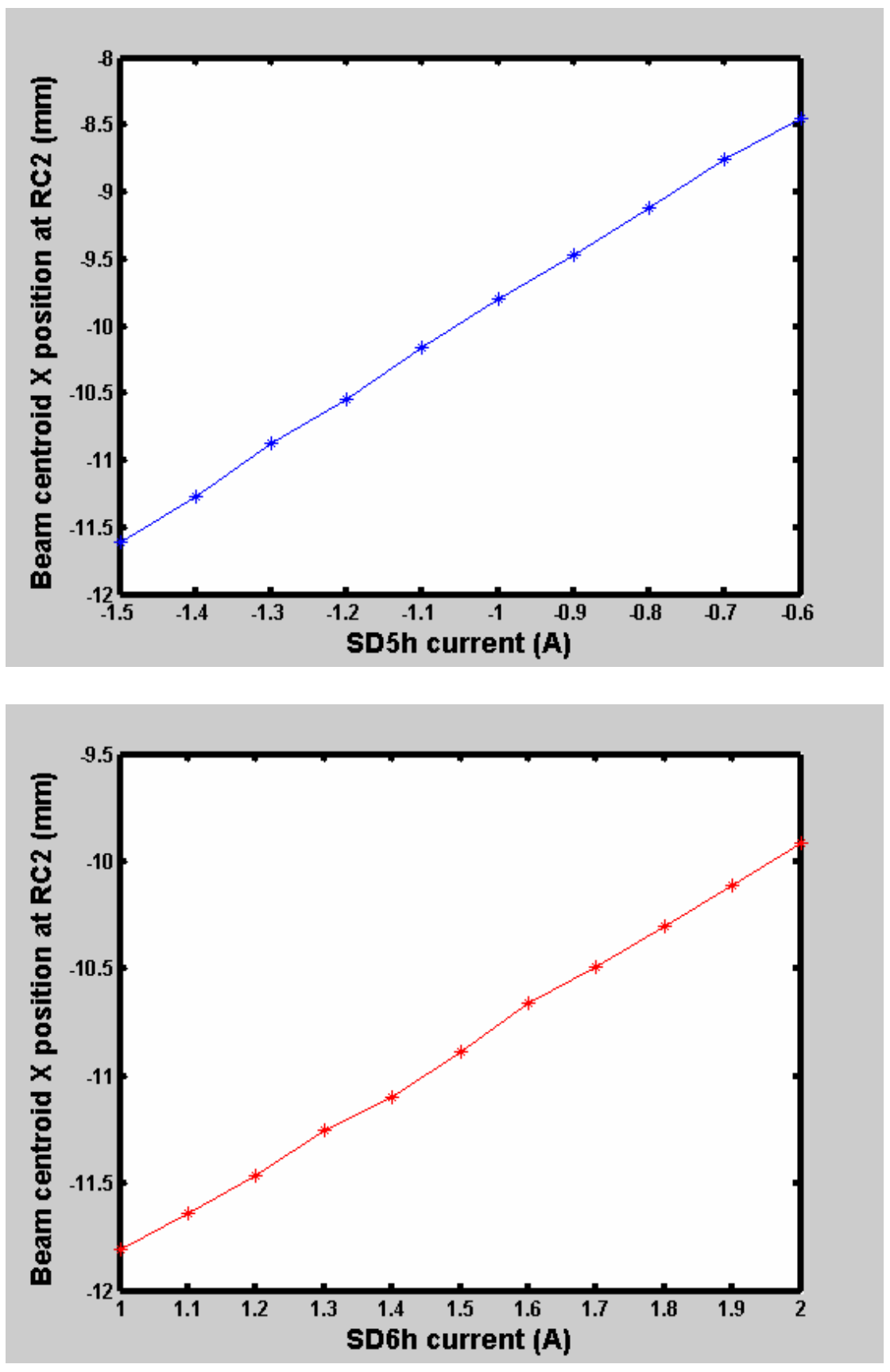

Figure 4.8 (a) Centroid sensitivity of the beam at RC2 due to the SD5h scan in Matlab calculation (b) Centroid sensitivity of the beam at RC2 due to the SD6h scan in Matlab calculation 


\subsubsection{WARP simulation study on the beam centroid sensitivity}

The WARP simulations discussed in Section 3.3.2 are run again with the initial beam centroid $\mathrm{X}$ position obtained from the experiment. To see the effect of space charge, both $0.6 \mathrm{~mA}$ beam model and $24 \mathrm{~mA}$ beam model are used. Typical UMER beam parameters are set in the two WARP models according to the parameters used in the experiment such as the beam kinetic energy $E_{0}$ is $10 \mathrm{keV}$; the pipe radius is equal to $2.54 \mathrm{~cm}$. Other beam parameters and numerical settings in this simulation are same with the WARP simulation in Section 3.3.2. For both of two cases, the initial beam centroid $\mathrm{X}$ position is set as $\mathrm{X}=-0.1741 \mathrm{~mm}$ acquired from the experiment.

With the procedure we used in Section 3.3.2, the strengths of SD5h and SD6h are scanned singly for both $0.6 \mathrm{~mA}$ and $24 \mathrm{~mA}$ beam models. Please refer the procedure details in Section 3.3.2. The results are shown in Figure 4.9. (a) shows the SD5h scan results: the blue circles represent $0.6 \mathrm{~mA}$ simulation and the green triangles represent $24 \mathrm{~mA}$ simulation; (b) is the scan results for SD6h; $0.6 \mathrm{~mA}$ simulation results are represented by the red circles and $24 \mathrm{~mA}$ results are represented by the blue triangles. The WARP scan results will be analyzed and compared with Matlab and experiment results in the following section. 

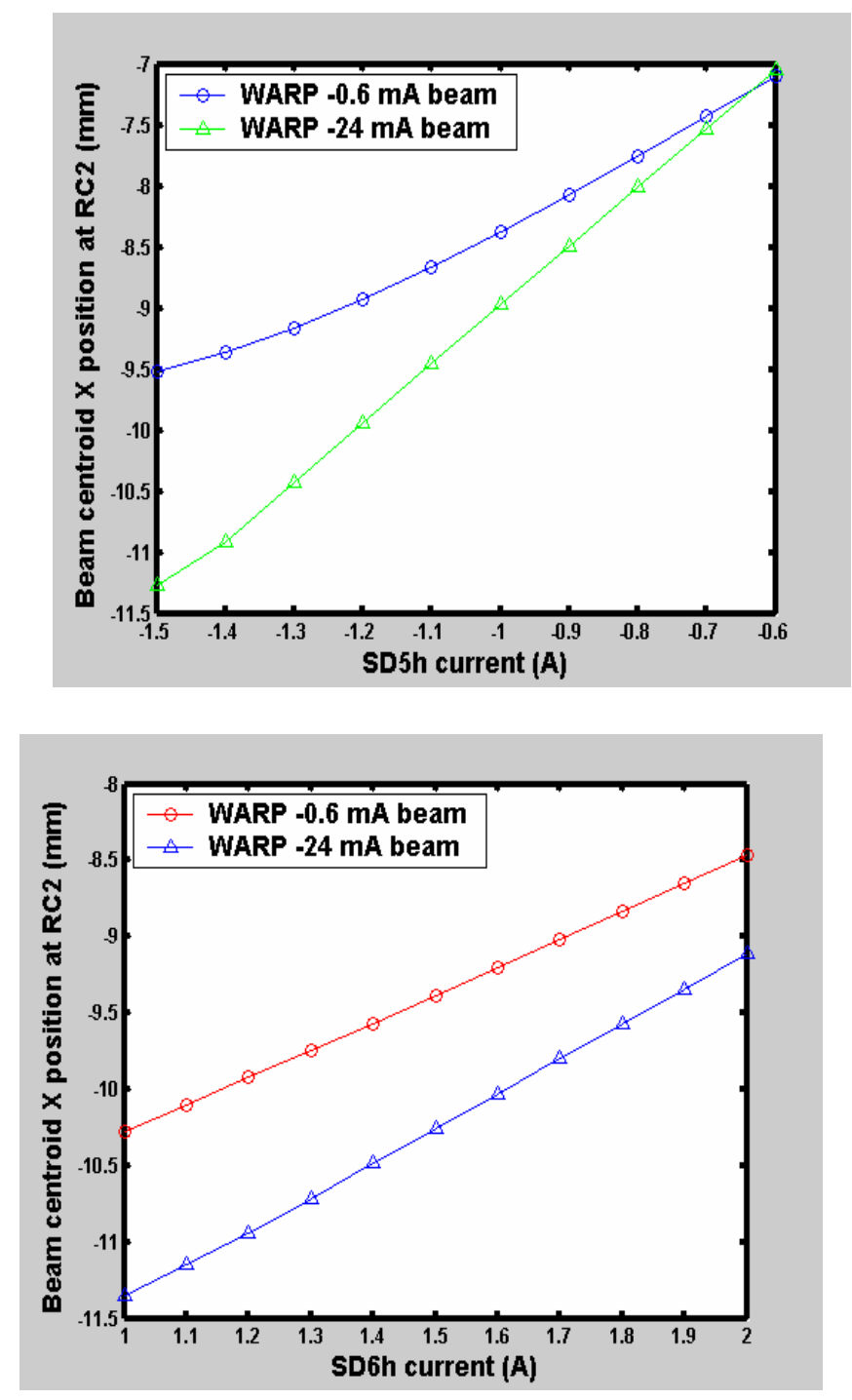

Figure 4.9 (a) Beam centroid sensitivities at RC2 due to the SD5h scan in WARP simulations (b) Beam centroid sensitivities at RC2 due to the SD6h scan in WARP simulations

\subsubsection{Analysis and comparison of experiment, Matlab, WARP simulation results}

The experiment results are analyzed and compared with simulation results from the Matlab model and both two WARP models in this section. Each set of results from the experiment, the Matlab simulation and the WARP simulation for both SD5h and SD6h are done by linear fit, respectively, to get the sensitivities defined as the fit slope. All sets of results from the experiment, Matlab and WARP 
simulations and corresponding best fit lines for SD5h scan are shown in Figure 4.10, as well as Figure 4.11 is for SD6h scan: the experiment results and best fit line are represented by the blue squares and the blue line; the Matlab results and best fit line are represented by the black stars and the black line; the $0.6 \mathrm{~mA}$ WARP results and best fit line are represented by the red circles and the red line; the $24 \mathrm{~mA}$ WARP results and best fit line are represented by the green triangles and the green line. The experiment results for both SD5h and SD6h are shifted by $-1 \mathrm{~cm}$ because what we are concerned with is the sensitivity. In the experiment, the absolute beam $\mathrm{X}$ centroid may be deflected greatly at RC2 because of some little errors. From all the best fit lines, good linearity of the sensitivities for both SD5h and SD6h scans can be observed.

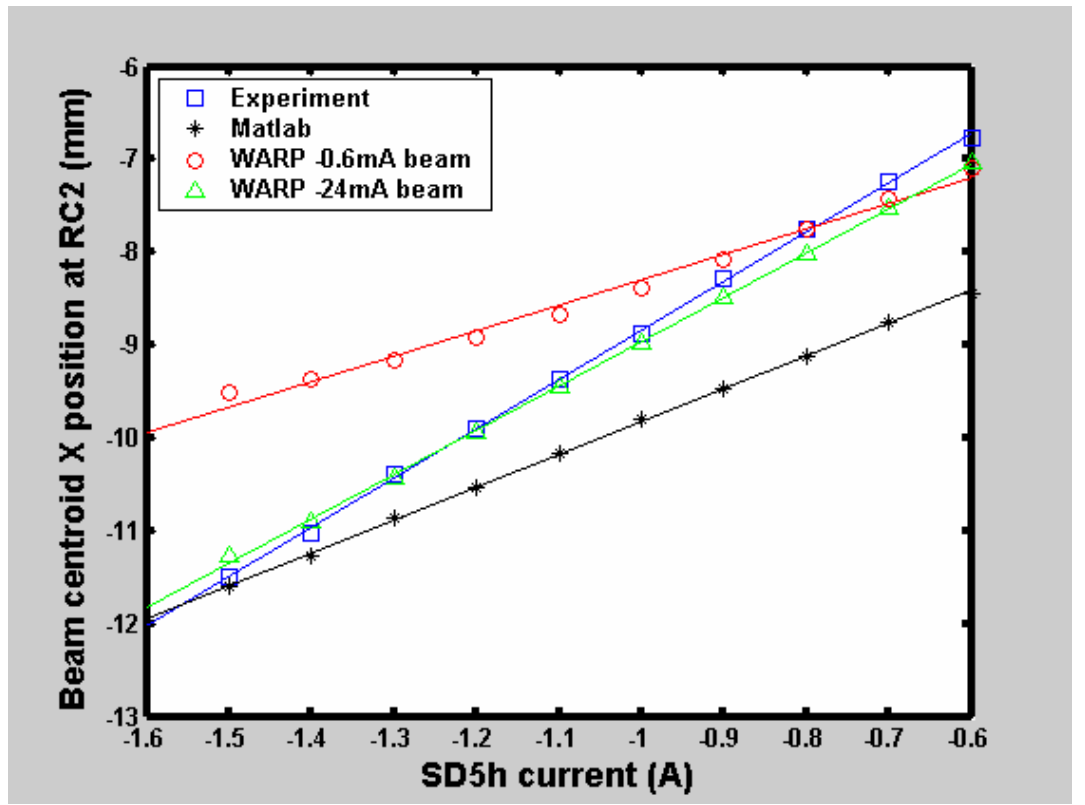

Figure 4.10 Best fit analysis and comparison of the beam centroid at RC2 due to SD5h scan among experiments, Matlab and WARP simulations 


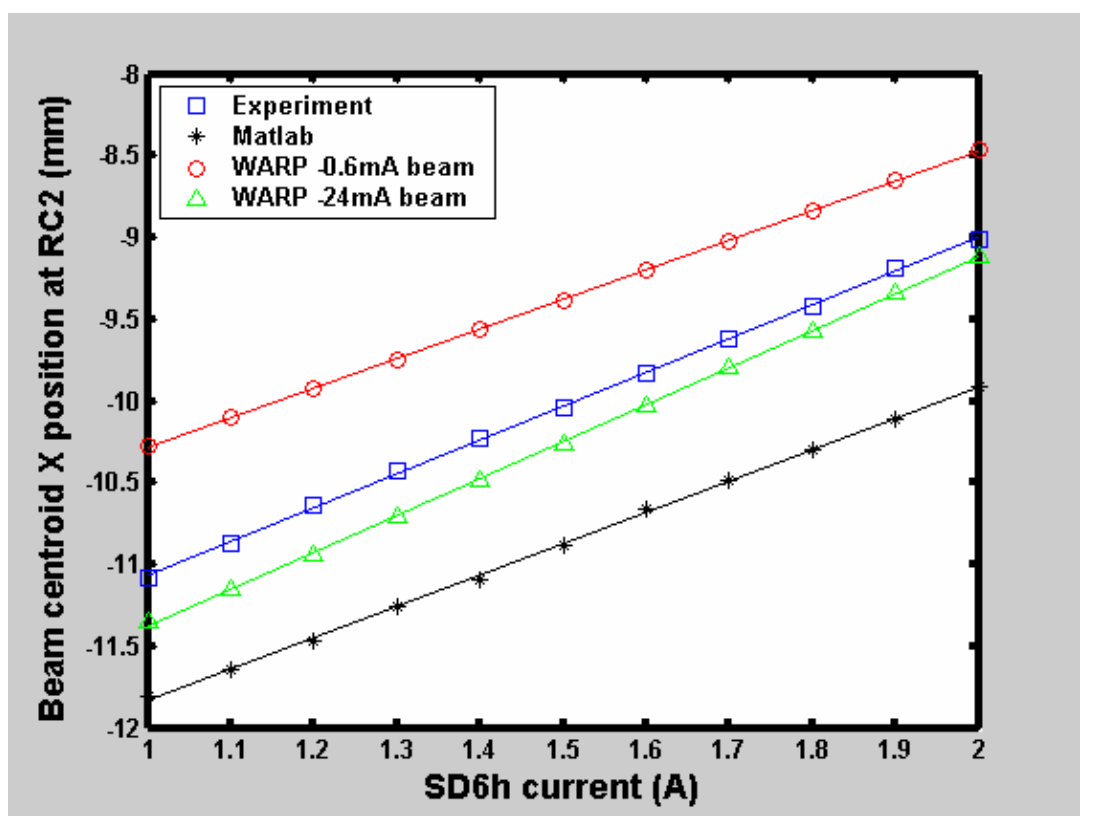

Figure 4.11 Best fit analysis and comparison of the beam centroid at RC2 due to SD6h scan among experiments, Matlab and WARP simulations

All the sensitivities for SD5h and SD6h acquired from the experiment, both Matlab and WARP simulations are shown in Table 4.7 and comparison is followed.

Table 4.7 Centroid sensitivities of beam at RC2 for SD5h and SD6h scans among experiments, Matlab and WAPR simulations

\begin{tabular}{|c|c|c|c|c|}
\hline & \multicolumn{2}{|c|}{ Space charge } & \multicolumn{2}{|c|}{ Non-space charge } \\
\hline & Experiment & 24mA-WARP & Matlab & $0.6 \mathrm{~mA}-\mathrm{WARP}$ \\
\hline SD5h sensitivity $(\mathrm{mm} / \mathrm{A})$ & 5.30 & 4.77 & 3.54 & 2.74 \\
\hline SD6h sensitivity $(\mathrm{mm} / \mathrm{A})$ & 2.07 & 2.26 & 1.92 & 1.81 \\
\hline
\end{tabular}

In experiment, the operating beam is a $24 \mathrm{~mA}$ beam with space charge. Thus the sensitivity obtained from the experiment is expected similar with the result from 24mA WARP simulation. As shown in Table 4.7, comparison between the 
sensitivities of the experiment and 24mA WARP simulation shows good agreements for both SD5h and SD6h.

Consistent with the simulation results of initial $\mathrm{Y}=-0.4 \mathrm{~mm}$ case, the sensitivities from Matlab (single particle) agree with $0.6 \mathrm{~mA}$ WARP results (contains no space charge) for SD5h and SD6h respectively.

In order to see if the space charge impacts the sensitivity, we divide the results in Table 4.7 into two groups: the space charge group is composed of the experiment and the 24mA WARP simulation, concerning with the space charge effect; the nonspace charge group includes the Matlab simulation and 0.6mA WARP simulation. As shown in Table 4.7, the sensitivities of the space charge group are larger than that of the non-space charge group for both SD5h and SD6h, respectively. This is consistent with the comparison result from initial $\mathrm{Y}=-0.4 \mathrm{~mm}$ case. Therefore it is reasonable to conclude that the space charge containing impacts the beam centroid sensitivity and more space charge results in a larger sensitivity. Due to the beam injection errors or lens misalignments, the beam centroid deflects from the pipe center and forms centroid coherent oscillation. When the beam contains space charge, the image charges appear. The image charge force impacts the centroid oscillation and sensitivity correspondingly. 


\section{Chapter 5 Conclusion}

The purpose of this thesis is to study the injection/recirculating of beam into a space-charge dominated ring, the University of Maryland Electron Ring through both mathematic modeling and experimental implementations.

The University of Maryland Electron Ring (UMER) is introduced first. UMER is designed as a small-scale simulator for beam physics research, which is a low-cost electron storage ring. Then some important issues of UMER design and key characters of UMER are discussed. Since the goal of UMER implementation is beam multi-turn operation, the most important issue is to correctly design the injection/recirculating section, called Y-section, which is a big challenge for the following reasons: UMER operation requires a very fast pulsed dipole, which is the critical component of the injection/recirculating section; this section consists of several big magnets in a very restricted space, forming a overlapped, complicated magnetic field; the earth's field has to be accounted for in the design because of the low energy beam; the space charge and image force also impacts the beam centroid motion.

After an analysis of the challenge, an improved model including the critical magnets in the injection/recirculating section is built to obtain basic design solutions for this section. A numerical calculation is developed in the Matlab to track the beam trajectories in this region for calculating the settings of the magnets in the model. The magnetic field modeling is modified due to the thickness of the pulsed dipole and the 
earth's field. The magnet settings for the different modeling are compared and indicate that: the magnet settings for the thick pulsed dipole model are almost same with that of the thin pulsed dipole model, meaning that the pulsed dipole can be modeled as a thin dipole in the calculation without affecting the results; the beam centroids for the two models with/without the earth's field are different, as well as the magnet settings different. As a result, the earth's field can not be ignored in the calculation due to the low energy electron beam.

After that, the studied section is extended to a more accurate and extended model, including the injection line, the Y-section and the first two sections of the ring. An important issue discussed here is accurately modeling of the magnetic field of the overlapped big magnets set in the stringent Y-section. A WARP model is built to calculate the complicated field and obtain the more accurate effective lengths.

With the single particle motion equation, a semi-analytic numerical algorithm is implemented based on the leap-frog iteration. Using this single particle numerical algorithm, the sensitivity of the beam centroid through the Y-section until RC2 is calculated in the Matlab. Also, with the same initial beam condition as the one used in the Matlab calculation, WARP simulations are executed to address the issue of the space charge effects. First, $0.6 \mathrm{~mA}$ beam assuming no space charge is used and the sensitivity shows a good agreement with the Matlab result; second, $24 \mathrm{~mA}$ beam concerning with the space charge is implemented and the sensitivity at RC2 is larger than the results of the Matlab and 0.6 mA WARP simulation.

A study of the settings of the pulsed dipole follows. This is a key issue for beam multi-turn operation. The pulsed dipole settings are calculated in the injection 
line and the returning leg, respectively. For each case, the beam centroid is tracked to pass through the Y-section and hit both centers of the QR1 and QR2, based on the numerical calculation in the more accurate model. The simulation solutions show good agreement with the experiment. The solutions from both the experiment and simulation show that: the pulsed dipole bends the beam towards the ring center for the returning case and switch to an opposite polarity to deflect the beam outwards the ring when the beam is injected. This is expected by the UMER Y-section design; the amplitude of the returning case is much smaller than that of the injection case, which is found due to the asymmetric effect of the earth's field on the beam centroid. The earth's field resists the pulsed dipole bending for the injecting beam but assists bending when the beam returns.

Finally, an experimental study is implemented to study the sensitivity of the beam centroid passing through the Y-section. The $24 \mathrm{~mA}$ beam is operated in the experiment. The initial beam condition obtained from the experiment is imported into the Matlab simulation and both $0.6 \mathrm{~mA}$ and $24 \mathrm{~mA}$ WARP simulations. All the results show good linearity of the beam centroid sensitivity. Comparison among the experimental and simulation results points out: the sensitivity calculated from the single particle Matlab simulation agrees with the result from $0.6 \mathrm{~mA}$ WARP simulation not containing the space charge; the $24 \mathrm{~mA}$ WARP simulation result, concerning with the space charge, shows a good agreement with the experimental result using $24 \mathrm{~mA}$ beam. The sensitivity obtained from the space charge case (the experiment and $24 \mathrm{~mA}$ WARP simulation) is larger than the non-space charge case (the Matlab and 0.6 mA WARP simulations) for both SD5h and SD6h, respectively. 
Conclusion can be made that the increased space charge impacts the beam centoid motion and sensitivity because of the image charge birth. 


\section{Bibliography}

[1] J. Wei, et al, "Spallation Neutron Source Ring - Status, Challenges, Issues, and Perspectives", Proc. IEEE 2003 PAC, Portland, OR, p. 571, 2003.

[2] T. C. Marshall, "Free Electron Laser,” Macmillan Pub. Co., New York, 1985.

[3] Proceedings of the 1986 Symposium on Heavy Ion Inertial Fusion, AIP, Conference Proceedings 152. New York, 1986.

[4] Proceedings of the 1988 International Symposium on Heavy Ion Inertial Fusion, GSI, Darmstadt, FRG, June 28-30, 1988, Nucl. Instr. Meth., A 278, 1, 1989.

[5] E. P. Lee, Nuovo Cimento, 106A, 1993.

[6] H. Li, "Control and transport of intense electron beams," Doctoral Dissertation, University of Maryland, 2004.

[7] P. G. O'Shea, et. al., "The University of Maryland Electron Ring (UMER)," Nuclear Instruments and Methods in Physics Research A 464, pp. 646-652, 2001.

[8] UMER website: http://www.ireap.umd.edu/umer .

[9] S. Bernal, et al, Commissioning of the University of Maryland Electron Ring (UMER), these proceedings, PAC'2005, Knoxville, TN

[10] R.A. Kishek, et al, Significance of the Earth Magnetic Field on Dispersive Characteristics of a Low Energy Electron Beam, PAC'2005, Knoxville, TN

[11] P. G. O’Shea, Personal Communication, Aug. 2004.

[12] D.P. Grote, et al, Fus. Eng. Des. 32-33,193-200, 1996.

[13] WARP online manual. 
[14] M. Reiser, "Theory and Design of Charged Particle Beams," Wiley \& Sons, New York, 1994.

[15] Y.J. Huo, "Experimental study of laser produced electron beam", Master Thesis, University of Maryland, 2004

[16] R.T. Avery, G.R. Lambertson, C.D. and Pike, Proc. IEEE 1971 PAC Conf., Chicago, IL, p. 885, 1971.

[17] M. Venturini, Ph.D. dissertation, Univ. of Maryland, College Park, 1998.

[18] Rawson-Lush Instrument Co. Inc., Acton, MA.

[19] W.W. Zhang, S. Bernal, H. Li, T. Godlove, R.A. Kishek, P.G. O'Shea, M. Reiser, V. Yun, and M. Venturini, "Design and Field measurement of PrintedCircuit Quadrupoles and Dipoles”, Phys. Rev. ST. Accel. Beams 3, 122401, 2000.

[20] H. Li, Master thesis, "Printed-Circuit Magnets System for University of Maryland Electron Ring”, Univ. of Maryland, College Park, 2001.

[21] B. Quinn, Measurements of the earth's magnetic field at the location of UMER [22] H. Li, et al, Beam Optics Design on a New Injection Scheme for UMER, PAC 2003, Portland, OR.

[23] S. Bernal, T. Godlove, G. Bai, Summary of UMER magnets.

[24] Cooperation with R.A. Kishek.

[25] B. Leblond, "Short pulse photoemission from a dispenser cathode under the 2nd, 3rd and 4th harmonics of a picosecond Nd:YAG laser," Nucl. Instr. Meth., A 317, pp. 365-372, 1992.

[26] Labview manual. 
[27] B. Quinn, Calibration of the BPMs operated by UMER.

[28] Cooperation with M. Walter. 